TRANSACTIONS OF THE

AMERICAN MATHEMATICAL SOCIETY

Volume 351, Number 2, February 1999, Pages 735-780

S 0002-9947(99)02168-6

\title{
ON SIEGEL MODULAR FORMS OF HALF-INTEGRAL WEIGHTS AND JACOBI FORMS
}

\author{
KOICHI TAKASE
}

\begin{abstract}
We will establish a bijective correspondence between the space of the cuspidal Jacobi forms and the space of the half-integral weight Siegel cusp forms which is compatible with the action of the Hecke operators. This correspondence is based on a bijective correspondence between the irreducible unitary representations of a two-fold covering group of a symplectic group and a Jacobi group (that is, a semidirect product of a symplectic group and a Heisenberg group). The classical results due to Eichler-Zagier and Ibukiyama will be reconsidered from our representation theoretic point of view.
\end{abstract}

\section{INTRODUCTION}

Jacobi forms are closely related with modular forms of half-integral weights. Such a relation is one of three main steps in the proof of Saito-Kurokawa lifting (other steps are the Fourier-Jacobi expansion of Siegel cusp forms in the Maass space, and the Shimura correspondence between integral and half-integral weight modular forms) [E-Z]. Similar relations between Jacobi forms of higher degree and Siegel modular forms of half-integral weights are studied by [Ibu]. The purpose of this paper is to study these relations from the representation theoretic point of view.

The basic idea is quite simple. For the sake of simplicity, we will consider the relations over the real number field (in this paper, we will work also over $p$-adic fields and over the adele ring). Let $(V, D)$ be a symplectic $\mathbb{R}$-space with a polarization $V=W \oplus W^{\prime}$ (that is, $D$ is a non-degenerate skew-symmetric $\mathbb{R}$-bilinear form on finite dimensional $\mathbb{R}$-vector space $V$, and both $W$ and $W^{\prime}$ are $\mathbb{R}$-vector subspaces of $V$ such that $\left.D(W, W)=D\left(W^{\prime}, W^{\prime}\right)=0\right)$. Let $L$ and $L^{\prime}$ be $\mathbb{Z}$-lattices of $W$ and $W^{\prime}$, respectively, such that $L \oplus L^{\prime}$ is the self-dual with respect to $D$. Let $G$ be a locally compact unimodular group and

$$
\rho: G \rightarrow S p(V)
$$

be a continuous group homomorphism. Let $\Gamma$ be a closed unimodular subgroup of $G$ such that $\rho(\Gamma) \subset S p(L)$ (see $\mathbf{1 . 2 . 4}$ for the notation). Let $\widetilde{S p}(V)$ be a nontrivial two-fold covering group of $S p(V)$ with covering mapping $\varpi$, and let $\widetilde{G}=$ $G \times{ }_{S p(V)} \widetilde{S p}(V)$ be a fiber product with

$$
\widetilde{\rho}: \widetilde{G} \rightarrow \widetilde{S p}(V), \quad \varpi_{G}: \widetilde{G} \rightarrow G
$$

Received by the editors February 5, 1997.

1991 Mathematics Subject Classification. Primary 11F37, 11F27; Secondary 11F70.

(C)1999 American Mathematical Society 
the canonical projections. Let $H[V]=V \times \mathbb{R}$ be the Heisenberg group associated with $(V, D)$ (see 1.2.1). The symplectic group $S p(V)$ acts on $H[V]$ as an automorphism group. Let $G_{J}=G \ltimes H[V]$ be the semi-direct product with respect to the action of $G$ on $H[V]$ via $\rho$. Put $\widetilde{G}_{J}=\widetilde{G} \ltimes H[V]$ where $\widetilde{G}$ acts on $H[V]$ via $\varpi_{G}$. Set $\widetilde{\Gamma}=\varpi_{G}^{-1}(\Gamma)$ and $\widetilde{S p}(L)=\varpi^{-1}(S p(L))$. Then $\widetilde{\rho}(\widetilde{\Gamma}) \subset \widetilde{S p}(L)$. Put $\Gamma_{J}=\Gamma \ltimes\left(L \oplus L^{\prime} \times \mathbb{R}\right)$ which is a closed unimodular subgroup of $G_{J}$.

Let $\omega$ be the Weil representation of $\widetilde{S p}(V)$ which is realized on $L^{2}(W)$. A unitary representation $\omega_{\rho}=\omega \circ \widetilde{\rho}$ is extended to an irreducible unitary representation of $\widetilde{G}_{J}$ which is also denoted by $\omega_{\rho}$. We will consider the simplest case of

$$
\left.\omega_{\rho}\right|_{Z(H[V])}=\mathbf{e}_{\infty}
$$

where $\mathbf{e}_{\infty}(t)=\exp 2 \pi \sqrt{-1} t$ is a character of $Z(H[V])=\mathbb{R}$. Take any unitary representation $\tau$ of $\widetilde{G}$. Let $\tau_{J}$ be the composition of $\tau$ with the canonical projection of $\widetilde{G}_{J}$ onto $\widetilde{G}$. Then the correspondence

$$
\tau \mapsto \pi=\tau_{J} \otimes \omega
$$

is a bijection between the unitary equivalence classes of the unitary representations $\tau$ of $\widetilde{G}$ and the unitary equivalence classes of the unitary representations $\pi$ of $\widetilde{G}_{J}$ such that $\left.\pi\right|_{Z(H[V])}=\mathbf{e}_{\infty}$. Also $\pi$ is irreducible if and only if $\tau$ is irreducible.

For example, let us consider an induced representation $\operatorname{Ind}\left(G_{J}, \Gamma_{J} ; \alpha \otimes \xi\right)$. Here $\alpha$ is a continuous unitary character (1-dimensional unitary representation) of $\Gamma$ and $\xi$ is a character of $\left(L \oplus L^{\prime}\right) \times \mathbb{R}$ defined by

$$
\xi(\lambda)=\mathbf{e}_{\infty}\left(t+\frac{1}{2} D(x, y)\right) \quad \text { for } \lambda=((x, y), t) \text { with } x \in L, y \in L^{\prime}, t \in \mathbb{R} .
$$

The character $\alpha \otimes \xi$ of $\Gamma_{J}$ is defined by

$$
(\alpha \otimes \xi)(\gamma, \lambda)=\alpha(\gamma) \xi(\lambda) .
$$

Considering $\operatorname{Ind}\left(G_{J}, \Gamma_{J} ; \alpha \otimes \xi\right)$ as a representation of $\widetilde{G}_{J}$ via its canonical projection onto $G_{J}$, we have

$$
\operatorname{Ind}\left(G_{J}, \Gamma_{J} ; \alpha \otimes \xi\right)=\operatorname{Ind}\left(\widetilde{G}, \widetilde{\Gamma} ; \varepsilon_{\Gamma}^{-1} \otimes \widetilde{\alpha}\right)_{J} \otimes \omega_{\rho} .
$$

Here $\widetilde{\alpha}$ is the composition of $\alpha$ with the covering mapping $\widetilde{\Gamma} \rightarrow \Gamma$, and $\varepsilon_{\Gamma}$ is a character of $\widetilde{\Gamma}$ defined as follows. Let us define a theta series $\theta_{\varphi}$ associated with a Schwartz function $\varphi \in \mathcal{S}(W)$ by

$$
\theta_{\varphi}(\sigma)=\sum_{\ell \in L}(\omega(\sigma) \varphi)(\ell) \quad \text { for all } \sigma \in \widetilde{S p}(V) .
$$

Then there exists a character $\varepsilon_{L, \infty}$ of $\widetilde{S p}(L)$ such that

$$
\theta_{\varphi}(\gamma \sigma)=\varepsilon_{L, \infty}(\gamma) \theta_{\varphi}(\sigma) \text { for all } \gamma \in \widetilde{S p}(L) .
$$

Put $\varepsilon_{\Gamma}=\varepsilon_{L, \infty} \circ \widetilde{\rho}$. See $\mathbf{1 . 2 . 4}$ for the details.

Let $\tau$ and $\pi$ be irreducible unitary representations of $\widetilde{G}$ and $G_{J}$, respectively, such that $\pi=\tau_{J} \otimes \check{\omega}_{\rho}$ where $\check{\omega}_{\rho}$ is the contragredient representation of $\omega_{\rho}$. Then (2) shows that the $\check{\pi}$-isotypic component $\operatorname{Ind}\left(G_{J}, \Gamma_{J} ; \alpha \otimes \xi ; \check{\pi}\right)$ of $\operatorname{Ind}\left(G_{J}, \Gamma_{J} ; \alpha \otimes \xi\right)$ corresponds to the $\check{\tau}$-isotypic component $\operatorname{Ind}\left(\widetilde{G}, \widetilde{\Gamma} ; \varepsilon_{\Gamma}^{-1} \otimes \widetilde{\alpha} ; \check{\tau}\right)$ of $\operatorname{Ind}\left(\widetilde{G}, \widetilde{\Gamma} ; \varepsilon_{\Gamma}^{-1} \otimes \widetilde{\alpha}\right)$. Roughly speaking, the space of automorphic forms on $G_{J}$ associated with $\pi$ corresponds to the space of the automorphic forms on $\widetilde{G}$ associated with $\tau$. How explicitly can the correspondence be described? 
Suppose that $G$ is a reductive real Lie group with maximal compact subgroup $K$ such that $G / K$ is a bounded symmetric domain, and $\rho$ is an $\left(H_{1}\right)$-group homomorphism (that is, the differential of $\rho$ is an $\left(H_{1}\right)$-homomorphism in the sense of [Sat5]). $\left(H_{1}\right)$-group homomorphisms are important for the study of the families of abelian varieties [Kug], [Mum], [Sat4]. In this case, if $\tau$ is a holomorphic discrete series of $\widetilde{G}$, then $\pi=\tau_{J} \otimes \check{\omega}_{\rho}$ is a "holomorphic discrete series" of $G_{J}$ (that is, a holomorphic induction on $G_{J}$ from $K \ltimes Z(H[V])$, see Remark 1.4.6).

Now let $(G, H)$ be a reductive dual pair in $S p(V)$ such that $H$ is compact and $\rho$ be the inclusion of $G$ into $S p(V)$. This is a typical example of an $\left(H_{1}\right)$-group homomorphism. Let $\tau$ be a holomorphic discrete series of $\widetilde{G}$ and put $\pi=\tau \otimes \check{\omega}_{\rho}$. We know that $\check{\omega}_{\rho}$ has a minimal $\widetilde{K}=\varpi_{G}^{-1}(K)$-type $\delta_{0}$ with multiplicity one $[\mathrm{K}-\mathrm{V}]$. Let $\delta^{\prime}$ be the minimal $\widetilde{K}$-type of $\tau$. Then $\pi$ has a minimal $K$-type $\delta=\delta^{\prime} \otimes \delta_{0}$ with multiplicity one. This is a phenomenon which is special for the holomorphic discrete series. Now the $\check{\delta}$-isotypic component of $\operatorname{Ind}\left(G_{J}, \Gamma_{J} ; \alpha \otimes \xi ; \check{\pi}\right)$ is the space of (generalized) Jacobi forms on $G_{J}$ of weight $\delta$, index 1 and with a character $\alpha$. On the other hand, the $\check{\delta}^{\prime}$-isotypic component of $\operatorname{Ind}\left(\widetilde{G}, \widetilde{\Gamma} ; \varepsilon_{\Gamma}^{-1} \otimes \widetilde{\alpha} ; \check{\tau}\right)$ is the space of holomorphic automorphic forms on $G$ of "half-integral weight" $\delta^{\prime}$ with a character $\varepsilon_{\Gamma}^{-1} \otimes \widetilde{\alpha}$. A minimal $\widetilde{K}$-type vector of $\omega_{\rho}$ produces a classical theta series. So a Jacobi form and a half-integral weight modular form correspond to each other by cutting off the theta series, or by integrating against the theta series. This is the correspondence studied by [E-Z] or [Ibu] (see §3.3). In this paper, we will consider the case of

$$
G=\text { symplectic group }, \quad H=\text { compact orthogonal group. }
$$

Other cases are treated similarly. Although we work only over the rational number field, the cases over the totally real number fields can be treated without any change in the arguments.

This paper consists of three chapters and two appendices.

In Chapter one, we will work over every local field $\mathbb{Q}_{p}(p \leq \infty)$. In $\S 1.1$, we will set up the fundamental framework of this paper. We will define a generalized Jacobi group and set the fundamental assumptions (A), (B) and (C) of 1.1.2. In $\S 1.2$, we will recall basic facts on the Weil representation. In $\S 1.3$, we will establish the correspondence (1) of unitary representations for our generalized Jacobi group (Theorem 1.3.3). In $\S 1.4$, we will work over the real number field $\mathbb{R}=\mathbb{Q}_{\infty}$. This case is connected directly with the classical treatment of automorphic forms. In fact, based upon the representation theoretic consideration described above, we will establish a bijection between cuspidal Jacobi forms (of degree $n$ with a matrix index) and Siegel cusp forms of half-integral weights of degree $n$ (Theorem 1.4.10).

In Chapter two, we will work only over finite local fields $\mathbb{Q}_{p}(p<\infty)$. The correspondence of automorphic forms given by [E-Z] or [Ibu] is compatible with the action of Hecke operators. This means that we need a $\mathbb{C}$-algebra isomorphism between the algebra $\mathcal{H}_{J, p}$ of Hecke operators on the finite local Jacobi group $G_{J, p}$ and the algebra $\mathcal{H}_{p}$ of Hecke operators on the finite local covering group $\widetilde{G}_{p}$ of $G_{p}$. Such an isomorphism was given by Shintani [Shn] (see Proposition 2.2.1). The $\mathbb{C}$-algebras $\mathcal{H}_{J, p}$ and $\mathcal{H}_{p}$ have canonical $\mathbb{C}$-bases which consist of the characteristic functions of suitable double cosets in $G_{J, p}$ and $\widetilde{G}_{p}$, respectively (see 2.1.4 and 2.1.5). We will show that the representation matrix, with respect to these canonical $\mathbb{C}$-bases, of the $\mathbb{C}$-algebra isomorphism given by Shintani is diagonal (Theorem 
2.3.2). A $\mathbb{C}$-algebra homomorphism between $\mathcal{H}_{J, p}$ and $\mathcal{H}_{p}$ reflects a correspondence between the zonal spherical functions on $G_{J, p}$ and on $\widetilde{G}_{p}$. This correspondence of zonal spherical functions contains the correspondence (1) for the class-one unitary representations of $G_{J, p}$ and of $\widetilde{G}_{p}$. Here, the zonal spherical function associated with the Weil representation studied in $\S 2.2$ plays the fundamental role. In particular, Proposition 2.2.2 is a key proposition of Chapter two. In $\S 2.4$, we will consider the class-one unitary representations of $G_{J, p}$ and $\widetilde{G}_{p}$. These results will be used in the global theory of Chapter three.

In Chapter three, we will work over the adele ring. We will establish the correspondence (1) of unitary representations and the identity (2) over the adele ring (or for the adelized groups). Then choosing carefully the local representations, we will show the global correspondence of automorphic forms (Theorem 3.1.10). In $\S 3.2$, we will consider the infinite local theory of $\S 1.4$ with regard to the Hecke operators. We will show that the infinite local correspondence of automorphic forms given in Theorem 1.4.8 (or Theorem 1.4.10 with classical terminology) is compatible with the action of Hecke operators (Theorem 3.2.6). Finally in $\S 3.3$, we will reconsider the result of $[\mathrm{E}-\mathrm{Z}]$ and $[\mathrm{Ibu}]$ from our point of view.

In the two appendices, we will recall some basic facts which are used throughout this paper. In Appendix A, we will recall some basic facts on the space of automorphic forms associated with an irreducible unitary representation of a locally compact unimodular group. See [Tak1] for the details. In Appendix B, we will recall basic facts on the zonal spherical functions with central character on a locally compact group.

Notation. The ring of the rational integers is denoted by $\mathbb{Z}$. The field of the rational numbers (resp. real numbers, complex numbers) is denoted by $\mathbb{Q}$ (resp. $\mathbb{R}, \mathbb{C}$ ).

For any finite or infinite place $p$ of $\mathbb{Q}$, the $p$-adic completion of $\mathbb{Q}$ is denoted by $\mathbb{Q}_{p}$. The closure of $\mathbb{Z}$ in $\mathbb{Q}_{p}$ is denoted by $\mathbb{Z}_{p}$ (so $\mathbb{Z}_{\infty}=\mathbb{Z}$ ). A Haar measure on $\mathbb{Q}_{p}$ is normalized so that $\operatorname{vol}\left(\mathbb{Z}_{p}\right)=1$ if $p<\infty, \operatorname{vol}([0,1])=1$ if $p=\infty$. The adele ring of $\mathbb{Q}$ is denoted by $\mathbb{Q}_{A}$. Let $\mathbf{e}=\bigotimes_{p} \mathbf{e}_{p}$ be the unique continuous character of $\mathbb{Q}_{A}$ which is trivial on $\mathbb{Q}$ and $\mathbf{e}_{\infty}(x)=\exp 2 \pi \sqrt{-1} x$. For a $\mathbb{Q}$-vector space $V$, put $V_{p}=V \otimes_{\mathbb{Q}} \mathbb{Q}_{p}$ and $V_{A}=V \otimes_{\mathbb{Q}} \mathbb{Q}_{A}$. For a $\mathbb{Z}$-module $M$, put $M_{p}=M \otimes_{\mathbb{Z}} \mathbb{Z}_{p}$.

For $K$-vector spaces $U$ and $V$ over a field $K$, we will denote by $\operatorname{Hom}_{K}(U, V)$ the $K$-vector space consisting of the $K$-linear mappings from $U$ to $V$. Set $\operatorname{End}_{K}(V)=$ $\operatorname{Hom}_{K}(V, V)$. For all $u \in U$ and $a \in \operatorname{Hom}_{K}(U, V)$, denoted by $u a \in V$ is the image of $u$ under $a$. Let us denote by $G L_{K}(V)$ the group of the $K$-linear isomorphisms of $V$ onto itself. Take $\mathbb{R}$-vector spaces $U, V$ and set $U_{\mathbb{C}}=U \otimes_{\mathbb{R}} \mathbb{C}$. The complex conjugation of $u \in U_{\mathbb{C}}$ over $U$ is denoted by $\bar{u}$. For any $a \in \operatorname{Hom}_{\mathbb{C}}\left(U_{\mathbb{C}}, V_{\mathbb{C}}\right)$, define $\bar{a} \in \operatorname{Hom}\left(U_{\mathbb{C}}, V_{\mathbb{C}}\right)$ by $u \bar{a}=\overline{\bar{u}} a$.

For a linear algebraic group $G$ over $\mathbb{Q}$, we will denote by $G(K)$ the group of the $K$-rational points for an over-field $K$ of $\mathbb{Q}$. Put $G_{\mathbb{Q}}=G(\mathbb{Q})$ and $G_{p}=G\left(\mathbb{Q}_{p}\right)$ $(p \leq \infty)$

For a topological space $X$, denoted by $C_{c}(X)$ is the complex vector space consisting of the compactly supported continuous $\mathbb{C}$-valued functions on $X$.

Throughout this paper, an induced representation is defined as follows. Let $G$ be a locally compact unimodular group and $H$ be a closed unimodular subgroup of $G$. Then the coset space $H \backslash G$ has a $G$-invariant measure $d \dot{g}$. Let $\xi$ be a unitary representation of $H$ with representation space $E_{\xi}$. Let $|\cdot|_{\xi}$ be the norm of the 
complex Hilbert space $E_{\xi}$. Let $C_{c}(G, H ; \xi)$ be a complex vector space consisting of the $H_{\xi}$-valued continuous functions $\varphi$ on $G$ such that

(1) $\varphi(h g)=\xi(h) \varphi(g)$ for all $h \in H$,

(2) the support of $\varphi$ is compact modulo $H$.

Then the induced representation $\pi=\operatorname{Ind}(G, H ; \xi)$ is the completion of $C_{c}(G, H ; \xi)$ with respect to the norm

$$
|\varphi|^{2}=\int_{H \backslash G}|\varphi(g)|_{\xi}^{2} d \dot{g} \quad \text { for } \varphi \in C_{c}(G, H ; \xi)
$$

with the action $(\pi(g) \varphi)(x)=\varphi(x g)$ for $\varphi \in C_{c}(G, H ; \xi)$ and $g \in G$.

\section{Chapter 1. Jacobi Group and its Unitary Representations}

\subsection{Jacobi group.}

1.1.1. Let $(V,[]$,$) be a symplectic \mathbb{Q}$-space with a polarization $V=W \oplus W^{\prime}$. An element $\sigma \in \operatorname{End}_{\mathbb{Q}}(V)$ is denoted by $\sigma=\left[\begin{array}{ll}a & b \\ c & d\end{array}\right]$ with $a \in \operatorname{End}_{\mathbb{Q}}(W), b \in$ $\operatorname{Hom}_{\mathbb{Q}}\left(W, W^{\prime}\right), c \in \operatorname{Hom}_{\mathbb{Q}}\left(W^{\prime}, W\right)$ and $d \in \operatorname{End}_{\mathbb{Q}}\left(W^{\prime}\right)$ such that $\left(w, w^{\prime}\right) \sigma=(w a+$ $\left.w^{\prime} c, w b+w^{\prime} d\right)$ for $(x, y) \in V=W \times W^{\prime}$. Let $(U,\langle\rangle$,$) be a regular quadratic \mathbb{Q}$-space. Let $L \subset W, L^{\prime} \subset W^{\prime}$ and $M \subset U$ be $\mathbb{Z}$-lattices such that $\left[L, L^{\prime}\right] \subset \mathbb{Z}$.

Put $\mathbb{V}=\operatorname{Hom}_{\mathbb{Q}}(U, V)$. For any $x \in \mathbb{V}$, there exists a unique ${ }^{\imath} x \in \operatorname{Hom}_{\mathbb{Q}}(V, U)$ such that

$$
\left\langle v \cdot{ }^{2} x, u\right\rangle=[v, u x], \quad \text { for all } v \in V, u \in U .
$$

For any $s \in \operatorname{End}_{\mathbb{Q}}(U)$, there exists a unique ${ }^{t} s \in \operatorname{End}_{\mathbb{Q}}(U)$ such that

$$
\left\langle u \cdot{ }^{t} s, u^{\prime}\right\rangle=\left\langle u, u^{\prime} s\right\rangle, \quad \text { for all } u, u^{\prime} \in U .
$$

Put

$$
\mathbb{U}=\operatorname{Sym}_{\mathbb{Q}}(U)=\left\{s \in \operatorname{End}_{\mathbb{Q}}(U) \mid{ }^{t} s=s\right\} .
$$

For all $x, y \in \mathbb{V}$, put $D(x, y)=\frac{1}{2}\left(x \cdot{ }^{\imath} y-y \cdot{ }^{\imath} x\right)$, which is an element of $\mathbb{U}$ because we have ${ }^{t}\left(x \cdot{ }^{\imath} y\right)=-y \cdot{ }^{\imath} x$. Then a group law on $H[\mathbb{V}, D]=\mathbb{V} \times \mathbb{U}$ is defined by

$$
(x, s) \cdot(y, t)=\left(x+y, s+t+\frac{1}{2} D(x, y)\right) .
$$

The center of $H[\mathbb{V}, D]$ is identified with $\mathbb{U}$ by $(0, s)=s$.

The group of the symplectic similitudes on $V$

$$
G S p(V)=\left\{\sigma \in G L_{\mathbb{Q}}(V) \mid[x \sigma, y \sigma]=\nu(\sigma)[x, y], \text { for all } x, y \in V, \nu(\sigma) \in \mathbb{Q}^{\times}\right\}
$$

acts on $H[\mathbb{V}, D]$ from the right as an automorphism group by $(x, s)^{\sigma}=(x \sigma, \nu(\sigma) s)$.

Let $G$ and $G_{J}$ be algebraic groups over $\mathbb{Q}$ such that

$$
G_{\mathbb{Q}}=S p(V)=\{\sigma \in G S p(V) \mid \nu(\sigma)=1\} \quad \text { and } \quad G_{J, \mathbb{Q}}=S p(V) \ltimes H[\mathbb{V}, D],
$$

respectively. The semi-direct product is defined by the action of $S p(V)$ on $H[\mathbb{V}, D]$ described above. We have an identification $Z\left(G_{J}\right)_{\mathbb{Q}}=\mathbb{U}$ by $(1,(0, s))=s$ for the center $Z\left(G_{J}\right)$ of $G_{J}$. 
1.1.2. Take an $S \in \mathbb{U}$ such that $\operatorname{det} S \neq 0$. Put $D_{S}(x, y)=\operatorname{tr}(S \cdot D(x, y))$. Then $D_{S}$ is a symplectic form on $\mathbb{V}$. Put

$$
\mathbb{W}=\operatorname{Hom}_{\mathbb{Q}}(U, W) \hookrightarrow \mathbb{V}, \quad \mathbb{W}^{\prime}=\operatorname{Hom}_{\mathbb{Q}}\left(U, W^{\prime}\right) \hookrightarrow \mathbb{V} .
$$

Then $\mathbb{V}=\mathbb{W} \oplus \mathbb{W}^{\prime}$ gives a polarization of $\mathbb{V}$ with respect to $D_{S}$, and $\langle x, y\rangle_{S}=$ $D_{S}(x, y)$ gives a non-degenerate pairing $\mathbb{W} \times \mathbb{W}^{\prime} \rightarrow \mathbb{Q}$. Put

$$
\mathcal{L}=\operatorname{Hom}_{\mathbb{Z}}(M, L) \hookrightarrow \mathbb{W}, \quad \mathcal{L}^{\prime}=\operatorname{Hom}_{\mathbb{Z}}\left(M, L^{\prime}\right) \hookrightarrow \mathbb{W}^{\prime}
$$

by the $\mathbb{Q}$-linear extension. Define a group homomorphism

$$
\rho: S p(V) \rightarrow S p(\mathbb{V}) \quad \text { by } x \rho(\sigma)=x \sigma .
$$

We will pose the following three conditions throughout this paper:

(A) there exists an ortho-normal $\mathbb{Q}$-basis of $U$ with respect to $\langle$,$\rangle ,$

(B) $S=P{ }^{t} P$ for some $P \in G L_{\mathbb{Q}}(U)$,

(C) $\mathcal{L}^{\prime}=\left\{y \in \mathbb{W} \mid\langle\mathcal{L}, y\rangle_{S} \subset \mathbb{Z}\right\}$.

The conditions (A) and (B) imply that there exists a $\mathbb{Q}$-basis $\left\{u_{1}, u_{2}, \cdots, u_{m}\right\}$ of $U$ such that $\left\langle u_{i} S^{-1}, u_{j}\right\rangle=\delta_{i j}$. We will fix such a $\mathbb{Q}$-basis $\left\{u_{1}, \cdots, u_{m}\right\}$ throughout this paper. Then we have

$$
\langle x, y\rangle_{S}=\sum_{j=1}^{m}\left[u_{j} x, u_{j} y\right] \quad \text { for all } x \in \mathbb{W}, y \in \mathbb{W}^{\prime} .
$$

1.1.3. We will denote by $M^{*}=\{u \in U \mid\langle u, M\rangle \subset \mathbb{Z}\}$ the dual lattice of $M$. Then we have

$$
\left(L \oplus L^{\prime}\right) \cdot{ }^{t} x \subset M^{*} \quad \text { for all } x \in \mathcal{L} \oplus \mathcal{L}^{\prime} .
$$

Put $\mathcal{M}=\left\{s \in \mathbb{U} \mid M s \subset M^{*}\right\}$ which is a $\mathbb{Z}$-lattice in $\mathbb{U}$. (1.1.3.1) implies that

$$
D(x, y) \in \frac{1}{2} \mathcal{M} \quad \text { for all } x, y \in \mathcal{L} \oplus \mathcal{L}^{\prime} .
$$

1.1.4. Fix $\mathbb{Z}$-bases $\left\{v_{1}, \cdots, v_{n}\right\}$ and $\left\{v_{1}^{\prime}, \cdots, v_{n}^{\prime}\right\}$ of $L$ and $L^{\prime}$, respectively, such that $\left[v_{i}, v_{j}^{\prime}\right]=\delta_{i j} e_{j}$ with $0<e_{j} \in \mathbb{Z}$ and $e_{j} \mid e_{j+1}$. Identify $V$ with the $\mathbb{Q}$-vector space of the row vectors $\mathbb{Q}^{2 n}$ with respect to the $\mathbb{Q}$-basis $\left\{v_{1}, \cdots, v_{n}, v_{1}^{\prime}, \cdots, v_{n}^{\prime}\right\}$ of $V$. Then

$$
D\left(v, v^{\prime}\right)=v J^{t} v^{\prime} \quad \text { with } J=\left[\begin{array}{cc}
0 & X \\
-X & 0
\end{array}\right], X=\left[\begin{array}{lll}
e_{1} & & \\
& \ddots & \\
& & e_{n}
\end{array}\right] .
$$

Fix a $\mathbb{Z}$-basis $\left\{u_{1}^{\prime}, \cdots, u_{m}^{\prime}\right\}$ of $M$, and put $S_{0}=\left(\left\langle u_{i}^{\prime}, u_{j}^{\prime}\right\rangle\right)_{i, j=1, \cdots, m}$. By means of the identification $U=\mathbb{Q}^{m}$ with respect to $\left\{u_{1}^{\prime}, \cdots, u_{m}^{\prime}\right\}$, we have an identification

$$
\mathbb{U}=S_{0} \operatorname{Sym}_{m}(\mathbb{Q})=\operatorname{Sym}_{m}(\mathbb{Q}) S_{0}^{-1}, \quad \mathcal{M}=\operatorname{Sym}_{m}(\mathbb{Z}) S_{0}^{-1} .
$$

Then the condition $(\mathrm{C})$ of $\mathbf{1 . 1 . 2}$ is equivalent to the condition

$$
S_{0}^{-1} S \cdot M_{m, n}(\mathbb{Z}) \cdot X=M_{m, n}(\mathbb{Z}) .
$$

Put $v_{i}^{*}=e_{i}^{-1} v_{i}^{\prime}$. Then $\left\{v_{1}^{*}, \cdots, v_{n}^{*}\right\}$ is a $\mathbb{Z}$-basis of $L^{*}=\left\{y \in W^{\prime} \mid\langle L, y\rangle \subset \mathbb{Z}\right\}$, the dual lattice of $L$. 
1.1.5. Take a finite place $p$ of $\mathbb{Q}$. Suppose that $\left\{u_{1}, \cdots, u_{m}\right\}$ is a $\mathbb{Z}_{p}$-basis of the $\mathbb{Z}_{p}$-lattice $M_{p}$. Then the representation matrix of $S$ with respect to $\left\{u_{1}, \cdots, u_{m}\right\}$ is equal to $S_{0}=\left(\left\langle u_{i}, u_{j}\right\rangle\right)_{i, j=1, \cdots, m}$. The condition (1.1.4.1), which is equivalent to the condition $(\mathrm{C})$ of $\mathbf{1 . 1 . 2}$, implies that $X \in G L_{n}\left(\mathbb{Z}_{p}\right)$. This means that $L_{p}^{*}=L_{p}^{\prime}$, or $\left\{v_{1}^{*}, \cdots, v_{n}^{*}\right\}$ is a $\mathbb{Z}_{p}$-basis of $L_{p}^{\prime}$. Under the same assumption, we have $M_{p}^{*}=$ $M_{p} S^{-1}$, and $\operatorname{tr}(S \cdot s) \in \mathbb{Z}_{p}$ for all $s \in \mathcal{M}_{p}$.

1.2. Weil representation. In this section, we will recall some basic facts on the Weil representation. The results are described for $S p(V)$. Similar notations are used for $S p(\mathbb{V})$.

1.2.1. Fix a finite or infinite place $p$ of $\mathbb{Q}$. The Haar measure $d_{W_{p}}(w)$ on $W_{p}$ (resp. $d_{W_{p}^{\prime}}\left(w^{\prime}\right)$ on $\left.W_{p}^{\prime}\right)$ is normalized so that $\operatorname{vol}\left(L_{p}\right)=1\left(\operatorname{resp} . \operatorname{vol}\left(L_{p}^{*}\right)=1\right)$ if $p<\infty, \operatorname{vol}\left(W_{p} / L_{p}\right)=1\left(\operatorname{resp} . \operatorname{vol}\left(W_{p}^{\prime} / L_{p}^{*}\right)=1\right)$ if $p=\infty$. A Haar measure on $V_{p}=W_{p} \times W_{p}^{\prime}$ is defined by $d_{V_{p}}\left(w, w^{\prime}\right)=d_{W_{p}}(w) d_{W_{p}^{\prime}}\left(w^{\prime}\right)$.

Put $H\left[V_{p}\right]=V_{p} \times \mathbb{Q}_{p}$ with a group law $(v, s) \cdot\left(v^{\prime}, t\right)=\left(v+v^{\prime}, s+t+\frac{1}{2}\left[v, v^{\prime}\right]\right)$. It is a locally compact unimodular group and is called the Heisenberg group associated with the symplectic $\mathbb{Q}_{p}$-space $\left(V_{p},[],\right)$. A Haar measure on $H\left[V_{p}\right]$ is defined by $d_{H\left[V_{p}\right]}(v, s)=d_{V_{p}}(v) d s$. The group of the symplectic similitudes $G S p\left(V_{p}\right)$ acts on $H\left[V_{p}\right]$ from the right by $(v, s)^{\sigma}=(v \sigma, \nu(\sigma) s)$ as an automorphism group.

1.2.2. The Heisenberg group $H\left[V_{p}\right]$ has a unique irreducible unitary representation $\Pi_{p}$ such that $\Pi_{p}(0, s)=\mathbf{e}_{p}(s)$ for all $s \in \mathbb{Q}_{p}$. In fact $\Pi_{p}$ is realized on $L^{2}\left(W_{p}\right)$ by

$$
\left(\Pi_{p}(h) \varphi\right)(w)=\varphi(w+x) \mathbf{e}_{p}\left(s+\frac{1}{2}[x, y]+[w, y]\right) \quad\left(\varphi \in L^{2}\left(W_{p}\right)\right)
$$

for $h=((x, y), s) \in H\left[V_{p}\right]\left(x \in W_{p}, y \in W_{p}^{\prime}\right)$. This realization of $\Pi_{p}$ on $L^{2}\left(W_{p}\right)$ is called the Schrödinger model. Let us denote by $\operatorname{Aut}\left(L^{2}\left(W_{p}\right)\right)$ the group of the unitary isomorphism of $L^{2}\left(W_{p}\right)$ onto itself. Then $\operatorname{Aut}\left(L^{2}\left(W_{p}\right)\right)$ is a Hausdorff topological group with respect to the weakest topology such that $\operatorname{Aut}\left(L^{2}\left(W_{p}\right)\right) \ni T \mapsto$ $T \varphi \in L^{2}\left(W_{p}\right)$ is continuous for all $\varphi \in L^{2}\left(W_{p}\right)$. Let $M p\left(V_{p}\right)$ be the closed subgroup of $G_{p} \times \operatorname{Aut}\left(L^{2}\left(W_{p}\right)\right)\left(G_{p}=S p\left(V_{p}\right)\right)$ consisting of the $(\sigma, T) \in G_{p} \times \operatorname{Aut}\left(L^{2}\left(W_{p}\right)\right)$ such that $T^{-1} \circ \Pi_{p}(h) \circ T=\Pi_{p}\left(h^{\sigma}\right)$ for all $h \in H\left[V_{p}\right]$. Then $M p\left(V_{p}\right)$ is a locally compact group [Igu]. There exists uniquely a continuous group homomorphism $\Psi_{p}: M p\left(V_{p}\right) \rightarrow \mathbb{C}^{1}$ such that

1) $\Psi_{p}(1, \lambda)=\lambda^{2}$ for all $\lambda \in \mathbb{C}^{1} \hookrightarrow \operatorname{Aut}\left(L^{2}\left(W_{p}\right)\right)$,

2) $\Psi_{p}(\mathbf{r}(\sigma))=(\operatorname{det} c,-1)_{p} \gamma_{p}(1)^{\operatorname{dim} V}$ for all $\sigma=\left[\begin{array}{ll}a & b \\ c & d\end{array}\right] \in G_{p}$ such that $\operatorname{det} c \neq$ 0 ,

where $(*, *)_{p}$ is the Hilbert symbol and $\operatorname{det} c=\operatorname{det}\left(\left[v_{i}^{*} c, v_{j}^{*}\right]\right)_{i, j=1,2, \cdots, n}$. Put $\mathbf{r}(\sigma)=$ $\left(\sigma, \mathbf{r}_{0}(\sigma)\right) \in M p\left(V_{p}\right)$ where

$$
\begin{aligned}
& \left(\mathbf{r}_{0}(\sigma) \varphi\right)(w) \\
& \quad=|\operatorname{det} c|_{p}^{1 / 2} \int_{W_{p}} \varphi\left(w a+w^{\prime} c\right) \mathbf{e}_{p}\left(\frac{1}{2}\left[w a+w^{\prime} c, w b+w^{\prime} d\right]-\frac{1}{2}\left[w, w^{\prime}\right]\right) d_{W_{p}^{\prime}}\left(w^{\prime}\right)
\end{aligned}
$$


for all $\varphi \in L^{2}\left(W_{p}\right) \cap L^{1}\left(W_{p}\right)$ [Wei]. The Weil number $\gamma_{p}(a)$ for $a \in \mathbb{Q}_{p}^{\times}$is defined as follows. If $p=\infty$, we put $\gamma_{p}(a)=\mathbf{e}_{p}\left(\frac{1}{8} \frac{a}{|a|}\right)$, and if $p<\infty$, we put $\gamma_{p}(a)=$ $\mathfrak{G}_{p}(a) /\left|\mathfrak{G}_{p}(a)\right|$ with $\mathfrak{G}_{p}(a)=\sum_{x \in \frac{1}{2} \mathbb{Z}_{p} / \mathbb{Z}_{p}} \mathbf{e}_{p}\left(a x^{2}\right)$.

Put $\widetilde{G}_{p}=\widetilde{S p}\left(V_{p}\right)=\operatorname{Ker}\left(\Psi_{p}\right)$ which is a locally compact unimodular group. Let $\varpi_{p}: \widetilde{G}_{p} \rightarrow G_{p}$ be the projection to the first factor. Then $\left(\widetilde{G}_{p}, \varpi_{p}\right)$ is a non-trivial two-fold covering group of $G_{p}$. Let us denote by $\widetilde{\sigma} \in \widetilde{G}_{p}$ an element such that $\varpi_{p}(\widetilde{\sigma})=\sigma \in G_{p}$. Put $E_{p}=\operatorname{Ker}\left(\varpi_{p}\right)=\{(1, \pm 1)\}$. The projection to the second factor $\omega_{p}: \widetilde{G} \rightarrow \operatorname{Aut}\left(L^{2}\left(W_{p}\right)\right)$ is a unitary representation of $\widetilde{G}_{p}$ on $L^{2}\left(W_{p}\right)$, which is called the Weil representation of $G_{p}=S p\left(V_{p}\right)$.

1.2.3. Put $H\left[L_{p}\right]=\left(L_{p} \oplus L_{p}^{\prime}\right) \times \mathbb{Q}_{p}$ which is a closed unimodular subgroup of $H\left[V_{p}\right]$. Define a unitary character $\xi_{p}$ of $H\left[L_{p}\right]$ by $\xi_{p}\left(\left(w, w^{\prime}\right), s\right)=\mathbf{e}_{p}\left(s+\frac{1}{2}\left[w, w^{\prime}\right]\right)$. The unitary representation $\left(\Pi_{p}, L^{2}\left(W_{p}\right)\right)$ of $H\left[V_{p}\right]$ is unitarily equivalent to the induced representation $\operatorname{Ind}\left(H\left[V_{p}\right], H\left[L_{p}\right] ; \xi_{p}\right)$. In fact, for any $\varphi \in C_{c}\left(W_{p}\right)$, put

$\Theta_{\varphi}(h)=\int_{L_{p}} \varphi(w+\ell) \mathbf{e}_{p}\left(s+\frac{1}{2}\left[w, w^{\prime}\right]+\left[\ell, w^{\prime}\right]\right) d_{L_{p}}(\ell)\left(h=\left(\left(w, w^{\prime}\right), s\right) \in H\left[V_{p}\right]\right)$,

where the Haar measure $d_{L_{p}}(\ell)$ on $L_{p}$ is normalized so that $\operatorname{vol}\left(L_{p}\right)=1$, if $p<\infty$, or the counting measure, if $p=\infty$. Then $\varphi \mapsto \Theta_{\varphi}$ is extended to a unitary isomorphism from $\left(\Pi_{p}, L^{2}\left(W_{p}\right)\right)$ to $\operatorname{Ind}\left(H\left[V_{p}\right], H\left[L_{p}\right] ; \xi_{p}\right)$. The realization of $\Pi_{p}$ as $\operatorname{Ind}\left(H\left[V_{p}\right], H\left[L_{p}\right] ; \xi_{p}\right)$ is called the lattice model.

1.2.4. Let $S p\left(L_{p}\right)$ be a group consisting of the $\gamma=\left[\begin{array}{ll}a & b \\ c & d\end{array}\right] \in S p\left(V_{p}\right)$ such that

1) $\left(L_{p} \oplus L_{p}^{\prime}\right) \gamma=L_{p} \oplus L_{p}^{\prime}$, and

2) $\left[w a+w^{\prime} c, w x+w^{\prime}\right] \equiv\left[w, w^{\prime}\right]\left(\bmod 2 \mathbb{Z}_{p}\right)$ for all $w \in L_{p}$ and $w^{\prime} \in L_{p}^{\prime}$.

For any $\gamma \in S p\left(L_{p}\right)$, define a unitary automorphism $\mathbf{r}_{L_{p}}(\gamma)$ of $\operatorname{Ind}\left(H\left[V_{p}\right], H\left[L_{p}\right] ; \xi_{p}\right)$ by

$$
\left(\mathbf{r}_{L_{p}}(\gamma) \varphi\right)(h)=\varphi\left(h^{\gamma}\right) .
$$

The automorphism $\mathbf{r}_{L_{p}}(\gamma)$ induces a unitary automorphism on $L^{2}\left(W_{p}\right)$ via the isomorphism given in 1.2.3, which is also denoted by $\mathbf{r}_{L_{p}}(\gamma)$. Then $\gamma \mapsto \mathbf{r}_{L_{p}}^{\prime}(\gamma)=$ $\left(\gamma, \mathbf{r}_{L_{p}}(\gamma)\right)$ is a continuous group homomorphism of $S p\left(L_{p}\right)$ to $M p\left(V_{p}\right)$. If $p$ is an odd finite place, we have $\widetilde{S p}\left(L_{p}\right)=\mathbf{r}_{L_{p}}^{\prime}\left(S p\left(L_{p}\right)\right) \subset \widetilde{G}_{p}$. For $p=2$ or $\infty$, put $\widetilde{S p}\left(L_{p}\right)=\varpi_{p}^{-1}\left(S p\left(L_{p}\right)\right) \subset \widetilde{G}_{p}$. Then $\widetilde{S p}\left(L_{p}\right)$ is an open compact subgroup of $\widetilde{G}_{p}$ for all finite $p$.

Let us denote by $\varepsilon_{L, p}$ the unitary character of $\widetilde{S p}\left(L_{p}\right)$ defined by

$$
\widetilde{\gamma}=\left(1, \varepsilon_{L, p}(\widetilde{\gamma})\right) \cdot \mathbf{r}_{L_{p}}^{\prime}(\gamma) \quad \text { for all } \widetilde{\gamma} \in \widetilde{S p}\left(L_{p}\right) .
$$

Note that $\varepsilon_{L, p}=1$ for all $p \neq 2, \infty$. In particular, we have

$$
\theta_{\varphi}(\widetilde{\gamma} \widetilde{\sigma})=\varepsilon_{L, \infty}(\widetilde{\gamma}) \theta_{\varphi}(\widetilde{\sigma}) \text { for all } \widetilde{\gamma} \in \widetilde{S p}(L), \widetilde{\sigma} \in \widetilde{S p}\left(V_{\infty}\right)
$$


and all Schwartz functions $\varphi \in \mathcal{S}\left(W_{\infty}\right)$. Here

$$
\theta_{\varphi}(\widetilde{\sigma})=\sum_{\ell \in L}\left(\omega_{\infty}(\widetilde{\sigma}) \varphi\right)(\ell) \quad\left(\widetilde{\sigma} \in \widetilde{S p}\left(V_{\infty}\right)\right)
$$

is the theta series associated with $\varphi \in \mathcal{S}\left(W_{\infty}\right)$.

1.2.5. Let

$$
H\left[V_{A}\right]=V_{A} \times \mathbb{Q}_{A}=\prod_{p \leq \infty}^{\prime} H\left[V_{p}\right]
$$

be the restricted direct product of $\left\{H\left[V_{p}\right]\right\}_{p \leq \infty}$ w.r.t. $\left\{\left(L_{p} \oplus L_{p}^{\prime}\right) \times \mathbb{Z}_{p}\right\}_{2<p<\infty}$. Let $\Pi$ be an irreducible unitary representation of $H\left[V_{A}\right]$ on $L^{2}\left(W_{A}\right)$ defined by

$$
(\Pi(h) \varphi)(w)=\varphi(w+x) \mathbf{e}\left(s+\frac{1}{2}[x, y]+[w, y]\right) \quad \text { for } h=((x, y), s) \in H\left[V_{A}\right] .
$$

Then $\Pi$ is unitarily equivalent to the restricted tensor product of $\left\{\Pi_{p}\right\}_{p \leq \infty}$ with respect to $\left\{\varphi_{L_{p}}\right\}_{p<\infty}$ where $\varphi_{L_{p}} \in L^{2}\left(W_{p}\right)$ is the characteristic function of $L_{p}$ in $W_{p}$, so we will identify them.

Let $M p\left(V_{A}\right)$ be the closed subgroup of $G_{A} \times \operatorname{Aut}\left(L^{2}\left(W_{A}\right)\right)$ consisting of $(\sigma, T) \in$ $G_{A} \times \operatorname{Aut}\left(L^{2}\left(W_{A}\right)\right)$ such that $T^{-1} \circ \Pi(h) \circ T=\Pi\left(h^{\sigma}\right)$ for all $h \in H\left[V_{A}\right]$. Then $M p\left(V_{A}\right)$ is a locally compact group [Igu].

Let $\widetilde{G}_{A}$ be the restricted direct product of $\left\{\widetilde{G}_{p}\right\}_{p \leq \infty}$ with respect to $\left\{\widetilde{S p}\left(L_{p}\right)\right\}_{p<\infty}$. Define a continuous group homomorphism

$$
J: \widetilde{G}_{A} \rightarrow M p\left(V_{A}\right) \quad \text { by } J\left(\left(\sigma_{p}, T_{p}\right)_{p \leq \infty}\right)=\left(\left(\sigma_{p}\right)_{p \leq \infty}, \bigotimes_{p \leq \infty} T_{p}\right) .
$$

Let $\widetilde{S p}\left(V_{A}\right)$ be the image of $J$ which is a closed normal subgroup of $M p\left(V_{A}\right)$ [Wei]. The projection to the second factor

$$
\omega_{A}: \widetilde{S p}\left(V_{A}\right) \rightarrow \operatorname{Aut}\left(L^{2}\left(W_{A}\right)\right)
$$

is a unitary representation of $\widetilde{S p}\left(V_{A}\right)$ which is called the global Weil representation.

$V \times \mathbb{Q}_{A}$ is a closed unimodular subgroup of $H\left[V_{A}\right]$. Define a unitary character $\xi_{A}$ of $V \times \mathbb{Q}_{A}$ by $\xi_{A}(v, s)=\mathbf{e}(s)$. Put

$$
\Theta_{\varphi}(h)=\sum_{\ell \in W} \varphi(w+\ell) \mathbf{e}\left(s+\frac{1}{2}\left[w, w^{\prime}\right]+\left[\ell, w^{\prime}\right]\right)
$$

for $h=\left(\left(w, w^{\prime}\right), s\right) \in H\left[V_{A}\right]$ and any Schwartz-Bruhat function $\varphi \in \mathcal{S}\left(W_{A}\right)$. Then $\varphi \mapsto \Theta_{\varphi}$ is extended to a unitary isomorphism from $\left(\Pi, L^{2}\left(W_{A}\right)\right)$ onto the induced representation $\operatorname{Ind}\left(H\left[V_{A}\right], V \times \mathbb{Q}_{A} ; \xi_{A}\right)$.

For any $\gamma \in S p(V)$, define a unitary automorphism $\mathbf{r}_{W}(\gamma)$ of $\operatorname{Ind}\left(H\left[V_{A}\right], V \times\right.$ $\left.\mathbb{Q}_{A} ; \xi_{A}\right)$ by $\left(\mathbf{r}_{W}(\gamma) \varphi\right)(h)=\varphi\left(h^{\gamma}\right)$. The automorphism $\mathbf{r}_{W}(\gamma)$ induces a unitary automorphism of $L^{2}\left(W_{A}\right)$ via the isomorphism defined above which is also denoted by $\mathbf{r}_{W}(\gamma)$. Then $\gamma \mapsto \mathbf{r}_{\mathbb{Q}}(\gamma)=\left(\gamma, \mathbf{r}_{W}(\gamma)\right)$ is a group homomorphism of $S p(V)$ into $\widetilde{S p}\left(V_{A}\right)[$ Wei]. Put

$$
\widetilde{S p}(V)=J^{-1}\left(\mathbf{r}_{\mathbb{Q}}(S p(V))\right)
$$

which is a discrete subgroup of $\widetilde{G}_{A}$. 
1.2.6. We have the following product formula.

\section{Proposition.}

$$
\prod_{p \leq \infty} \varepsilon_{p}\left(\gamma, T_{p}\right)=1 \text { for all }\left(\gamma, T_{p}\right)_{p \leq \infty} \in \widetilde{S p}(V) \cap\left(\widetilde{S p}\left(V_{\infty}\right) \times \prod_{p<\infty} \widetilde{S p}\left(L_{p}\right)\right) .
$$

Proof. For any Schwartz-Bruhat function $\varphi \in \mathcal{S}\left(W_{A}\right)$, put

$$
\theta_{\varphi}(s)=\sum_{w \in W}\left(\omega_{A}(s) \varphi\right)(w) \quad \text { for all } s \in \widetilde{S p}\left(V_{A}\right) .
$$

Then we have [Wei]

$$
\theta_{\varphi}\left(\mathbf{r}_{\mathbb{Q}}(\gamma) s\right)=\theta_{\varphi}(s) \quad \text { for all } \gamma \in S p(V), s \in \widetilde{S p}\left(V_{A}\right) .
$$

If we put $\theta^{\varphi}(\widetilde{\sigma})=\theta_{\varphi}(J(\widetilde{\sigma}))$ for $\widetilde{\sigma} \in \widetilde{G}_{A}$, then we have

$$
\theta^{\varphi}(\widetilde{\gamma} \widetilde{\sigma})=\theta^{\varphi}(\widetilde{\sigma}) \text { for all } \widetilde{\gamma} \in \widetilde{S p}(V) .
$$

Now set $\varphi=\bigotimes_{p \leq \infty} \varphi_{p}$ where $\varphi_{\infty} \in \mathcal{S}\left(W_{\infty}\right)$ is a Schwartz function and, for all $p<\infty, \varphi_{p}$ is the characteristic function of $L_{p}$ in $W_{p}$. Then

$$
\theta^{\varphi}(\widetilde{\sigma}, 1,1, \cdots)=\theta_{\varphi_{\infty}}(\widetilde{\sigma}) \text { for all } \widetilde{\sigma} \in \widetilde{S p}\left(V_{\infty}\right)
$$

(see 1.2.4). Take any

$$
\widetilde{\gamma}=\left(\gamma, T_{p}\right)_{p \leq \infty} \in \widetilde{S p}(V) \cap\left(\widetilde{S p}\left(V_{\infty}\right) \times \prod_{p<\infty} \widetilde{S p}\left(L_{p}\right)\right) .
$$

By (1.2.4.1), we have

$$
\theta_{\varphi_{\infty}}\left(\left(\gamma, T_{\infty}\right) \widetilde{\sigma}\right)=\varepsilon_{L, \infty}\left(\gamma, T_{\infty}\right) \theta_{\varphi_{\infty}}(\widetilde{\sigma}) \quad \text { for all } \widetilde{\sigma} \in \widetilde{S p}\left(V_{\infty}\right) .
$$

On the other hand, we have

$$
\begin{aligned}
\theta_{\varphi_{\infty}}\left(\left(\gamma, T_{\infty}\right) \widetilde{\sigma}\right) & =\theta^{\varphi}\left(\widetilde{\gamma} \cdot\left(\widetilde{\sigma},\left(\gamma, T_{p}\right)_{p<\infty}^{-1}\right)\right)=\theta^{\varphi}\left(\widetilde{\sigma},\left(\gamma, T_{p}\right)_{p<\infty}^{-1}\right) \\
& =\sum_{w \in W}\left(\omega_{\infty}(\widetilde{\sigma}) \varphi_{\infty}\right)(w) \cdot \prod_{p<\infty}\left(\omega_{p}\left(\gamma, T_{p}\right) \varphi_{p}\right)(w) \\
& =\prod_{p<\infty} \varepsilon_{L, p}\left(\gamma, T_{p}\right) \cdot \theta_{\varphi_{\infty}}(\widetilde{\sigma}) .
\end{aligned}
$$

Then we get the required product formula.

\subsection{Unitary representation of Jacobi group.}

1.3.1. Fix a finite or infinite place $p$ of $\mathbb{Q}$. We will denote by $H_{S}\left[\mathbb{V}_{p}\right]$ the Heisenberg group associated with the symplectic space $\left(\mathbb{V}_{p}, D_{S}\right)$. We have a surjective group homomorphism $(x, t) \mapsto(x, \operatorname{tr}(S \cdot t))$ of $H\left[\mathbb{V}_{p}, D\right]$ onto $H_{S}\left[\mathbb{V}_{p}\right]$. Then $H\left[\mathbb{V}_{p}, D\right]$ has a unique irreducible unitary representation $\Pi_{S, p}$ such that $\Pi_{S, p}(0, t)=\mathbf{e}_{p}(\operatorname{tr}(S \cdot t))$ for all $t \in \mathbb{U}_{p}$. Let $H_{p}$ be the representation space of $\Pi_{S, p}$. $\Pi_{S, p}$ is realized on $H_{p}=L^{2}\left(\mathbb{W}_{p}\right)$ by

$$
\left(\Pi_{S, p}(h) \varphi\right)(w)=\mathbf{e}_{p}\left(\operatorname{tr}(S \cdot t)+\frac{1}{2}\langle x, y\rangle_{S}+\langle w, y\rangle_{S}\right) \varphi(w+x) \quad\left(\varphi \in L^{2}\left(\mathbb{W}_{p}\right)\right)
$$


for $h=((x, y), t) \in H\left[\mathbb{V}_{p}, D\right]\left(x \in \mathbb{W}_{p}, y \in \mathbb{W}_{p}^{\prime}\right)$. It is unitarily isomorphic to $H_{p}=\operatorname{Ind}\left(H\left[\mathbb{V}_{p}, D\right], \Lambda_{p} ; \xi_{S, p}\right)$ where $\Lambda_{p}=\left(\mathcal{L}_{p} \oplus \mathcal{L}_{p}^{\prime}\right) \times \mathbb{U}_{p}$ and

$$
\xi_{S, p}(\lambda)=\mathbf{e}_{p}\left(\operatorname{tr}(S \cdot t)+\frac{1}{2}\langle x, y\rangle_{S}\right)
$$

$\left(\lambda=((x, y), t) \in \Lambda_{p}\right)$ is a unitary character of $\Lambda_{p}$. The unitary intertwining mapping $\varphi \mapsto \Theta_{\varphi}$ is given by

$$
\Theta_{\varphi}(h)=\int_{\mathcal{L}_{p}} \varphi(x+\ell) \mathbf{e}_{p}\left(\operatorname{tr}(S \cdot t)+\frac{1}{2}\langle x, y\rangle_{S}+\langle\ell, y\rangle_{S}\right) d_{\mathcal{L}_{p}}(\ell)
$$

for any $\varphi \in C_{c}\left(\mathbb{W}_{p}\right)$ and $h=((x, y), t) \in H\left[\mathbb{V}_{p}, D\right]$. The Weil representation $\omega_{p}$ of $S p\left(\mathbb{V}_{p}\right)$ is realized on $H_{p}$. Define a character $\chi_{S, p}$ of $Z\left(H\left[\mathbb{V}_{p}, D\right]\right)=\mathbb{U}_{p}$ by $\chi_{S, p}(t)=\mathbf{e}_{p}(\operatorname{tr}(S \cdot t))$ for $t \in \mathbb{U}_{p}$.

1.3.2. We shall define a continuous group homomorphism

$$
\widetilde{\rho}_{p}: \widetilde{G}_{p} \rightarrow \widetilde{S p}\left(\mathbb{V}_{p}\right) .
$$

First of all, identify $L^{2}\left(\mathbb{W}_{p}\right)$ with the completed $m$-fold tensor product $\widehat{\bigotimes}^{m} L^{2}\left(W_{p}\right)$ by

$$
\left(\bigotimes_{j=1}^{m} \varphi_{j}\right)(x)=\prod_{j=1}^{m} \varphi_{j}\left(u_{j} x\right) \quad \text { for } \varphi_{j} \in L^{2}\left(W_{p}\right), x \in \mathbb{W}_{p}
$$

Because of (1.1.2.1), we have a continuous group homomorphism $(\sigma, T) \mapsto(\rho(\sigma)$, $\left.T^{\otimes m}\right)$ of $M p\left(V_{p}\right)$ to $M p\left(\mathbb{V}_{p}\right)$. Using (1.1.2.1) again, we have

$$
\mathbf{r}_{0}(\rho(\sigma))=\mathbf{r}_{0}(\sigma)^{\otimes m} \quad \text { for all } \sigma=\left[\begin{array}{ll}
a & b \\
c & d
\end{array}\right] \in S p\left(V_{p}\right) \text { such that } \operatorname{det} c \neq 0 .
$$

Then we have $\Psi_{p}(\mathbf{r}(\rho(\sigma)))=\Psi_{p}(\mathbf{r}(\sigma))^{m}$. Now we will define the group homomorphism

$$
\widetilde{\rho}_{p}: \widetilde{G}_{p} \rightarrow \widetilde{S p}\left(\mathbb{V}_{p}\right) \text { by } \widetilde{\rho}_{p}(\sigma, T)=\left(\rho(\sigma), T^{\otimes m}\right) .
$$

1.3.3. Put $\widetilde{G}_{J, p}=\widetilde{G}_{p} \ltimes H\left[\mathbb{V}_{p}, D\right]$, where $\widetilde{G}_{p}$ acts on $H\left[\mathbb{V}_{p}, D\right]$ via the covering map $\varpi_{p}: \widetilde{G}_{p} \rightarrow G_{p}$. Define an irreducible unitary representation $\omega_{S, p}$ of $\widetilde{G}_{J, p}$ by $\omega_{S, p}(\widetilde{\sigma}, h)=\omega_{p}\left(\widetilde{\rho}_{p}(\widetilde{\sigma})\right) \circ \Pi_{S, p}(h)\left(\widetilde{\sigma} \in \widetilde{G}_{p}\right.$ and $\left.h \in H\left[\mathbb{V}_{p}, D\right]\right)$.

For any unitary representation $\pi$ of $\widetilde{G}_{p}$, let us denote by $\pi_{J}$ the composition of $\pi$ with the canonical projection of $\widetilde{G}_{J, p}$ onto $\widetilde{G}_{p}$. Then we have

Theorem. $\pi \mapsto \pi_{J} \otimes \omega_{S, p}$ gives a bijection between the set of the unitary equivalence classes of the unitary representations of $\widetilde{G}_{p}$ and the set of the unitary equivalence classes of the unitary representations $\tau$ of $\widetilde{G}_{J, p}$ such that $\tau(t)=\chi_{S, p}(t)$ for all $t \in Z\left(\widetilde{G}_{J, p}\right)=\mathbb{U}_{p} . \quad \pi_{J} \otimes \omega_{S, p}$ is irreducible if and only if $\pi$ is. $\pi_{J} \otimes \omega_{S, p}$ is square-integrable modulo the center if and only if $\pi$ is square-integrable.

This theorem is proved by an argument similar to that in [Sat2].

Remark. The unitary representations of $G_{J, p}$ are regarded as unitary representations of $\widetilde{G}_{J, p}$ via the canonical projection $\widetilde{G}_{J, p} \rightarrow G_{J, p}$. Then $\pi \mapsto \pi_{J} \otimes \omega_{S, p}$ gives a bijection between the set of the unitary equivalence classes of the unitary representations $\pi$ of $\widetilde{G}_{p}$ such that $\left.\pi\right|_{E_{p}}=\nu_{p}^{m}$ and the set of the unitary equivalence 
classes of the unitary representations $\tau$ of $G_{J, p}$ such that $\tau(t)=\chi_{S, p}(t)$ for all $t \in Z\left(G_{J, p}\right)=\mathbb{U}_{p}$.

1.4. Infinite local theory. In this section, we will develop our theory over $\mathbb{R}=$ $\mathbb{Q}_{\infty}$

1.4.1. Let $\Gamma$ be a discrete subgroup of $G_{\infty}=S p\left(V_{\infty}\right)$ such that $\rho(\Gamma) \subset S p(\mathcal{L})$. An example of such a discrete subgroup is given in 1.4.11. Let $\alpha$ be a unitary character of $\Gamma$. Put $\widetilde{\Gamma}=\varpi_{\infty}^{-1}(\Gamma) \subset \widetilde{G}_{\infty}$. Then $\widetilde{\rho}_{\infty}(\widetilde{\Gamma}) \subset \widetilde{S p}(\mathcal{L})$. The invariant measure on $\widetilde{\Gamma} \backslash \widetilde{G}_{\infty}$ is induced from the invariant measure on $\Gamma \backslash G_{\infty}$ via the topological isomorphism $\widetilde{\Gamma} \backslash \widetilde{G}_{\infty} \tilde{\rightarrow} \Gamma \backslash G_{\infty}$. A closed unimodular subgroup $\Lambda_{\infty}=\left(\mathcal{L} \oplus \mathcal{L}^{\prime}\right) \times \mathbb{U}_{\infty}$ of $H\left[\mathbb{V}_{\infty}, D\right]$ is stable under the action of $\Gamma$ via $\rho_{\infty}: G_{\infty} \rightarrow S p\left(\mathbb{V}_{\infty}\right)$. The semi-direct product $\Gamma_{J}=\Gamma \ltimes \Lambda_{\infty}$ is a closed unimodular subgroup of $G_{J, \infty}=$ $S p\left(V_{\infty}\right) \ltimes H\left[\mathbb{V}_{\infty}, D\right]$. Define a unitary character $\alpha \otimes \xi_{S, \infty}$ of $\Gamma_{J}$ by

$$
\left(\alpha \otimes \xi_{S, \infty}\right)(\gamma, \lambda)=\alpha(\gamma) \cdot \xi_{S, \infty}(\lambda) .
$$

Consider the induced representation $\pi=\operatorname{Ind}\left(G_{J, \infty}, \Gamma_{J} ; \alpha \otimes \xi_{S, \infty}\right)$. Let us denote by $\widetilde{\pi}$ and $\widetilde{\alpha}$ the composition of $\pi$ and $\alpha$ with the canonical projections $\widetilde{G}_{J, \infty} \rightarrow$ $G_{J, \infty}$ and $\widetilde{\Gamma} \rightarrow \Gamma$, respectively. Then we have $\widetilde{\pi}=\operatorname{Ind}\left(\widetilde{G}_{J, \infty}, \widetilde{\Gamma}_{J} ; \widetilde{\alpha} \otimes \xi_{S, \infty}\right)$. Put $\tau=\operatorname{Ind}\left(\widetilde{G}_{\infty}, \widetilde{\Gamma} ; \widetilde{\alpha} \otimes \varepsilon_{\Gamma}^{-1}\right)$ where $\varepsilon_{\Gamma}$ is the composition of $\varepsilon_{\mathcal{L}, \infty}$ with $\widetilde{\rho}$ restricted to $\widetilde{\Gamma}$ (see 1.2.4 for the definition of $\varepsilon_{\mathcal{L}, \infty}$ ). Then we have

Theorem. $\tau_{J} \otimes \omega_{S, \infty}$ is unitarily equivalent to $\widetilde{\pi}$ by the unitary mapping $\varphi \otimes \psi \mapsto$ $\varphi \otimes \psi$ defined by

$$
(\varphi \otimes \psi)(\sigma, h)=(\tau(\sigma) \varphi)(1) \cdot\left(\omega_{S, \infty}(\sigma, h) \psi\right)(1) .
$$

Here $\omega_{S, \infty}$ is realized on $\operatorname{Ind}\left(H\left[\mathbb{V}_{\infty}, D\right], \Lambda_{\infty} ; \xi_{S, \infty}\right)$.

Proof. By a direct calculation, it is easy to see that the $\mathbb{C}$-linear mapping $\varphi \otimes \psi \mapsto$ $\varphi \otimes \psi$ is $G_{J, \infty}$-equivariant and $|\varphi \otimes \psi|=|\varphi| \cdot|\psi|$. Put

$$
\theta_{\widetilde{\alpha} \otimes \xi_{S, \infty}, f}(g)=\int_{\widetilde{\Gamma}_{J}}\left(\widetilde{\alpha} \otimes \xi_{S, \infty}\right)\left(\gamma^{-1}\right) f(\gamma g) d \gamma \quad \text { for } f \in C_{c}\left(\widetilde{G}_{J, \infty}\right) .
$$

For any $f_{1} \in C_{c}\left(\widetilde{G}_{\infty}\right)$ and $f_{2} \in C_{c}\left(H\left[\mathbb{V}_{\infty}, D\right]\right)$, define

$$
f=f_{1} \otimes f_{2} \in C_{c}\left(\widetilde{G}_{J, \infty}\right) \quad \text { by } f(\sigma, h)=f_{1}(\sigma) \cdot f_{2}\left(h^{\sigma^{-1}}\right) .
$$

Then the $\mathbb{C}$-linear span of

$$
\left\{\theta_{\tilde{\alpha} \otimes \xi_{S, \infty}, f_{1} \otimes f_{2}} \mid f_{1} \in C_{c}\left(\widetilde{G}_{\infty}\right), f_{2} \in C_{c}\left(H\left[\mathbb{V}_{\infty}, D\right]\right)\right\}
$$

is dense in $\operatorname{Ind}\left(\widetilde{G}_{J, \infty}, \widetilde{\Gamma}_{J} ; \widetilde{\alpha} \otimes \xi_{S, \infty}\right)$. For any $\varphi \in C_{c}\left(\widetilde{G}_{\infty}\right)$ and $\psi \in C_{c}\left(H\left[\mathbb{V}_{\infty}, D\right]\right)$, put

$$
\theta_{\widetilde{\alpha} \otimes \varepsilon_{\Gamma}^{-1}, \varphi}(\sigma)=\int_{\widetilde{\Gamma}}\left(\widetilde{\alpha} \otimes \varepsilon_{\Gamma}^{-1}\right)(\gamma)^{-1} \varphi(\gamma \sigma) d \gamma \quad\left(\sigma \in \widetilde{G}_{\infty}\right)
$$

and

$$
\theta_{\xi_{S, \infty}, \psi}(h)=\int_{\Lambda_{\infty}} \xi_{S, \infty}(\lambda)^{-1} \psi(\lambda h) d \lambda \quad\left(h \in H\left[\mathbb{V}_{\infty}, D\right]\right),
$$

respectively. Then the $\mathbb{C}$-linear spans of

$$
\left\{\theta_{\widetilde{\alpha} \otimes \varepsilon_{\Gamma}^{-1}, \varphi} \mid \varphi \in C_{c}\left(\widetilde{G}_{\infty}\right)\right\} \quad \text { and } \quad\left\{\theta_{\xi_{S, \infty}, \psi} \mid \psi \in C_{c}\left(H\left[\mathbb{V}_{\infty}, D\right]\right)\right\},
$$


are dense in

$$
\operatorname{Ind}\left(\widetilde{G}_{\infty}, \widetilde{\Gamma} ; \widetilde{\alpha} \otimes \varepsilon_{\Gamma}^{-1}\right) \quad \text { and } \quad \operatorname{Ind}\left(H\left[\mathbb{V}_{\infty}, \Lambda_{\infty} ; \xi_{S, \infty}\right]\right)
$$

respectively. Take any $f=f_{1} \otimes f_{2}\left(f_{1} \in C_{c}\left(\widetilde{G}_{\infty}\right), f_{2} \in C_{c}(H[\mathbb{V} \infty, D])\right)$. Then, for any $g=\left(\sigma, h^{\sigma}\right) \in \widetilde{G}_{J, \infty}$, we have

$$
\begin{aligned}
\theta_{\widetilde{\alpha} \otimes \xi_{S, \infty}, f}(g) & =\int_{\widetilde{\Gamma}} d \gamma \int_{\Lambda_{\infty}} d \lambda \widetilde{\alpha}(\gamma)^{-1} \xi_{S, \infty}(\lambda)^{-1} f_{1}(\gamma \sigma) f_{2}\left((\lambda h)^{\gamma^{-1}}\right) \\
& =\int_{\widetilde{\Gamma}} d \gamma \widetilde{\alpha}(\gamma)^{-1} f_{1}(\gamma \sigma) \theta_{\xi_{S, \infty}, f_{2}}\left(h^{\gamma^{-1}}\right) \\
& =\int_{\widetilde{\Gamma}} d \gamma\left(\widetilde{\alpha} \otimes \varepsilon_{\Gamma}^{-1}\right)\left(\gamma^{-1}\right) f_{1}(\gamma \sigma)\left(\omega_{S, \infty}\left(\gamma^{-1}\right) \theta_{\xi_{S, \infty}, f_{2}}\right)(h) \\
& =\left(\omega_{S, \infty}(\sigma) \theta_{f, \sigma}\right)(h)
\end{aligned}
$$

where

$$
\theta_{f, \sigma}(h)=\int_{\widetilde{\Gamma}} d \gamma\left(\widetilde{\alpha} \otimes \varepsilon_{\Gamma}^{-1}\right)\left(\gamma^{-1}\right) f_{1}(\gamma \sigma)\left(\omega_{S, \infty}(\gamma \sigma)^{-1} \theta_{\xi_{S, \infty}, f_{2}}\right)(h) \quad\left(h \in H\left[\mathbb{V}_{\infty}, D\right]\right)
$$

is an element of $\operatorname{Ind}\left(H\left[\mathbb{V}_{\infty}, D\right], \Lambda_{\infty} ; \xi_{S, \infty}\right)$. On the other hand, we have

$$
\left(\theta_{\widetilde{\alpha} \otimes \varepsilon_{\Gamma}^{-1}, \varphi} \otimes \theta_{\xi_{S, \infty}, \psi}, \theta_{\widetilde{\alpha} \otimes \xi_{S, \infty}, f}\right)=\int_{\widetilde{G}_{\infty}} d \sigma \int_{H\left[\mathbb{V}_{\infty}, D\right]} d h \varphi(\sigma) \psi(h) \overline{\theta_{f, \sigma}(h)} .
$$

In fact, we have

$$
\begin{aligned}
& \left(\theta_{\widetilde{\alpha} \otimes \varepsilon_{\Gamma}^{-1}, \varphi} \otimes \theta_{\xi_{S, \infty}, \psi}, \theta_{\widetilde{\alpha} \otimes \xi_{S, \infty}, f}\right) \\
& =\int_{\widetilde{\Gamma} \backslash \widetilde{G}_{\infty}} d \sigma \int_{\Lambda_{\infty} \backslash H\left[\mathbb{V}_{\infty}, D\right]} d h \int_{\widetilde{\Gamma}} d \gamma \theta_{\widetilde{\alpha} \otimes \varepsilon_{\Gamma}^{-1}, \varphi}(\sigma)\left(\omega_{S, \infty}(\sigma) \theta_{\xi_{S, \infty}, \psi}\right)\left(h^{\sigma^{-1}}\right) \\
& =\int_{\widetilde{\Gamma} \backslash \widetilde{G}_{\infty}} d \sigma \int_{\widetilde{\Gamma}} d \gamma \theta_{\widetilde{\alpha} \otimes \varepsilon_{\Gamma}^{-1}, \varphi}(\gamma \sigma) \overline{f_{1}(\gamma \sigma)} \cdot\left(\omega_{\Gamma, \infty}^{-1}\right)(\gamma) \overline{f_{1}(\gamma \sigma)} \overline{\left(\omega_{S, \infty}\left(\gamma^{-1}\right) \theta_{\xi_{S, \infty}, f_{2}}\right)\left(h^{\sigma^{-1}}\right)} \\
& \left.=\int_{\widetilde{G}_{\infty}} d \sigma \theta_{\widetilde{\alpha} \otimes \varepsilon_{\Gamma}^{-1}, \varphi}, \theta_{\xi_{S, \infty}, f_{2}}\right) \\
&
\end{aligned}
$$

Here we have

$$
\begin{aligned}
& \left(\omega_{S, \infty}(\sigma) \theta_{\xi_{S, \infty}, \psi}, \theta_{\xi_{S, \infty}, f_{2}}\right)=\left(\theta_{\xi_{S, \infty}, \psi}, \omega_{S, \infty}(\sigma)^{-1} \theta_{\xi_{S, \infty}, f_{2}}\right) \\
& =\int_{\Lambda_{\infty} \backslash H\left[\mathbb{V}_{\infty}, D\right]} d h \int_{\Lambda_{\infty}} d \lambda \psi(\lambda h) \overline{\left(\omega_{S, \infty}(\sigma)^{-1} \theta_{\xi_{S, \infty}, f_{2}}\right)(\lambda h)} \\
& =\int_{H\left[\mathbb{V}_{\infty}, D\right]} d h \psi(h) \overline{\left(\omega_{S, \infty}(\sigma)^{-1} \theta_{\xi_{S, \infty}, f_{2}}\right)(h)} .
\end{aligned}
$$

Now we have

$$
\begin{aligned}
& \left(\theta_{\widetilde{\alpha} \otimes \varepsilon_{\Gamma}^{-1}, \varphi} \otimes \theta_{\xi_{S, \infty}, \psi}, \theta_{\widetilde{\alpha} \otimes \xi_{S, \infty}, f}\right) \\
& =\int_{\widetilde{G}_{\infty}} d \sigma \int_{H\left[\mathbb{V}_{\infty}, D\right]} d h \int_{\widetilde{\Gamma}} d \gamma \\
& \quad \cdot\left(\widetilde{\alpha} \otimes \varepsilon_{\Gamma}^{-1}\right)(\gamma)^{-1} \varphi(\gamma \sigma) \overline{f_{1}(\sigma)} \psi(h) \overline{\left(\omega_{S, \infty}(\sigma)^{-1} \theta_{\xi_{S, \infty}, f_{2}}\right)(h)} \\
& =\int_{\widetilde{G}_{\infty}} d \sigma \int_{H\left[\mathbb{V}_{\infty}, D\right]} d h \varphi(\sigma) \psi(h) \overline{\theta_{f, \sigma}(h)},
\end{aligned}
$$


which is the required formula. Suppose that

$$
\left(\theta_{\widetilde{\alpha} \otimes \varepsilon_{\Gamma}^{-1}, \varphi} \otimes \theta_{\xi_{S, \infty}, \psi}, \theta_{\widetilde{\alpha} \otimes \xi_{S, \infty}, f}\right)=0 \quad \text { for all } \varphi \in C_{c}\left(\widetilde{G}_{\infty}\right), \psi \in C_{c}\left(H\left[\mathbb{V}_{\infty}, D\right]\right) .
$$

Then $\theta_{f, \sigma}$ is a zero vector in $\operatorname{Ind}\left(H\left[\mathbb{V}_{\infty}, D\right], \Lambda_{\infty} ; \xi_{S, \infty}\right)$ for all $\sigma \in \widetilde{G}_{\infty}$. Hence $\theta_{\tilde{\alpha} \otimes \xi_{S, \infty}, f}=0$. So we have an isomorphism

$$
\operatorname{Ind}\left(\widetilde{G}_{\infty}, \widetilde{\Gamma} ; \widetilde{\alpha} \otimes \varepsilon_{\Gamma}^{-1}\right)_{J} \otimes \operatorname{Ind}\left(H\left[\mathbb{V}_{\infty}, D\right], \Lambda_{\infty} ; \xi_{S, \infty}\right) \stackrel{\sim}{\rightarrow} \operatorname{Ind}\left(\widetilde{G}_{J, \infty}, \widetilde{\Gamma}_{J} ; \widetilde{\alpha} \otimes \xi_{S, \infty}\right) .
$$

1.4.2. The Siegel upper half space associated with the polarization $V=W \oplus W^{\prime}$ is defined by

$$
\mathfrak{H}_{W}=\left\{z \in \operatorname{Sym}_{\mathbb{C}}\left(W_{\mathbb{C}}, W_{\mathbb{C}}^{\prime}\right) \mid \operatorname{Im}(z) \in \operatorname{Sym}_{\mathbb{R}}^{+}\left(W_{\infty}, W_{\infty}^{\prime}\right)\right\} .
$$

Here $W_{\mathbb{C}}=W_{\infty} \otimes_{\mathbb{R}} \mathbb{C}, \operatorname{Sym}_{\mathbb{C}}\left(W_{\mathbb{C}}, W_{\mathbb{C}}^{\prime}\right)$ is a $\mathbb{C}$-vector space of the $z \in \operatorname{Hom}_{\mathbb{C}}\left(W_{\mathbb{C}}, W_{\mathbb{C}}^{\prime}\right)$ such that $[v, w z]=[w, v z]$ for all $v, w \in W_{\mathbb{C}}$. We denote by $\operatorname{Sym}_{\mathbb{R}}^{+}\left(W_{\infty}, W_{\infty}^{\prime}\right)$ the open convex cone of the $s \in \operatorname{Sym}_{\mathbb{C}}\left(W_{\mathbb{C}}, W_{\mathbb{C}}^{\prime}\right)$ such that $W_{\infty} s \subset W_{\infty}^{\prime}$ and $[w, w s]>0$ for all $0 \neq w \in W_{\infty} . G_{J, \infty}$ acts transitively on $\mathfrak{H}_{W, J}=\mathfrak{H}_{W} \times \mathbb{W}_{\mathbb{C}}^{\prime}$ by

$$
g(Z)=\left(\sigma(z),(w+x z+y) J(\sigma, z)^{-1}\right)
$$

for $g=(\sigma, h) \in G_{J, \infty}$ with $h=((x, y), t) \in H\left[\mathbb{V}_{\infty}, D\right]$ and $Z=(z, w) \in \mathfrak{H}_{W, J}$. Here $\sigma(z)=(a z+b)(c z+d)^{-1}$ and $J(\sigma, z)=c z+d \in G L_{\mathbb{C}}\left(W_{\mathbb{C}}^{\prime}\right)$ for $\sigma=\left[\begin{array}{ll}a & b \\ c & d\end{array}\right] \in S p\left(V_{\infty}\right)$ as usual.

A $G_{J, \infty}$-invariant measure $d(z, w)=d(z) d_{z}(w)$ on $\mathfrak{H}_{W, J}$ is defined as follows:

$$
d(z)=\operatorname{det}(\operatorname{Im} z)^{-(n+1)} \prod_{1 \leq i<j \leq n} d\left(\left[v_{i}, v_{j} \operatorname{Re} z\right]\right) \prod_{1 \leq i<j \leq n} d\left(\left[v_{i}, v_{j} \operatorname{Im} z\right]\right),
$$

where $\operatorname{det}(\operatorname{Im} z)=\operatorname{det}\left(\left[v_{i}, v_{j} \operatorname{Im} z\right]\right)_{i, j=1, \cdots, n}$ is the $G_{\infty}$-invariant measure on $\mathfrak{H}_{W}$ and

$$
d_{z}(w)=\operatorname{det}(\operatorname{Im} z)^{-m} d_{\mathbb{W}_{\infty}^{\prime}}(\operatorname{Re} w) d_{\mathbb{W}_{\infty}^{\prime}}(\operatorname{Im} w)
$$

We have

$$
d_{z^{\prime}}\left(w^{\prime}\right)=d_{z}(w) \quad \text { for }\left(z^{\prime}, w^{\prime}\right)=g(z, w) \text { with } g \in G_{J, \infty} .
$$

We have also

$$
d_{z}(x z+y)=d_{\mathbb{W}_{\infty}}(x) d_{\mathbb{W}_{\infty}^{\prime}}(y) \quad \text { for all } x \in \mathbb{W}_{\infty}, y \in \mathbb{W}_{\infty}^{\prime}
$$

Put

$$
\begin{aligned}
\eta_{S}(g ; Z)=\mathbf{e}_{\infty}\left\{\operatorname{tr}(S \cdot t)+\frac{1}{2}\langle x, x \sigma(z)+y\rangle_{S}+\left\langle x, w J(\sigma, z)^{-1}\right\rangle_{S}\right. \\
\left.-\frac{1}{2}\left\langle w^{t} c, w J(\sigma, z)^{-1}\right\rangle_{S}\right\}
\end{aligned}
$$

for $g=\left(\sigma, h^{\sigma}\right) \in G_{J, \infty}$ with $h=((x, y), t) \in H\left[\mathbb{V}_{\infty}, D\right], \sigma=\left[\begin{array}{ll}a & b \\ c & d\end{array}\right] \in G_{\infty}$ and $Z=(z, w) \in \mathfrak{H}_{W, J}$. Then $\eta_{S}(g ; Z)$ is a factor of automorphy:

$$
\eta_{S}\left(g g^{\prime} ; Z\right)=\eta_{S}\left(g ; g^{\prime}(Z)\right) \eta_{S}\left(g^{\prime} ; Z\right) \text { for all } g, g^{\prime} \in G_{J, \infty} \text {. }
$$


Put

$$
\kappa_{S}\left(Z^{\prime}, Z\right)=\mathbf{e}_{\infty}\left\{\frac{1}{2}\left\langle\left(w^{\prime}-\bar{w}\right)\left(z^{\prime}-\bar{z}\right)^{-1}, w^{\prime}-\bar{w}\right\rangle_{S}\right\}
$$

for $Z=(z, w), Z^{\prime}=\left(z^{\prime}, w^{\prime}\right) \in \mathfrak{H}_{W, J}$. Then we have

$$
\kappa_{S}\left(g\left(Z^{\prime}\right), g(Z)\right)=\eta_{S}\left(g ; Z^{\prime}\right) \kappa_{S}\left(Z^{\prime}, Z\right) \overline{\eta_{S}(g ; Z)}
$$

for all $g \in G_{J, \infty}$. Put $\kappa_{S}(Z)=\kappa_{S}(Z, Z)$.

1.4.3. Fix a $z_{0} \in \mathfrak{H}_{W}$ which defines a maximal compact subgroup

$$
K_{\infty}=\left\{\sigma \in G_{\infty} \mid \sigma\left(z_{0}\right)=z_{0}\right\} \quad \text { of } G_{\infty} .
$$

Put $\beta_{W}(\sigma, \tau)=\varepsilon_{W}\left(\sigma ; z_{0}, \tau\left(z_{0}\right)\right)$ for all $\sigma, \tau \in G_{\infty}$ where

$\varepsilon_{W}\left(\sigma ; z^{\prime}, z\right)=\operatorname{det}^{-1 / 2}\left(\frac{\sigma\left(z^{\prime}\right)-\overline{\sigma(z)}}{2 \sqrt{-1}}\right) \cdot \operatorname{det}^{1 / 2}\left(\frac{z^{\prime}-\bar{z}}{2 \sqrt{-1}}\right) \cdot\left|\operatorname{det} J\left(\sigma, z^{\prime}\right) J(\sigma, z)\right|^{-1 / 2}$

for $\sigma \in G_{\infty}$ and $z, z^{\prime} \in \mathfrak{H}_{W}$. Here we put

$$
\operatorname{det}^{-1 / 2} T=\int_{W_{\infty}} \exp (-\pi[w, w T]) d_{W_{\infty}}(w)
$$

for all $T \in \operatorname{Sym}_{\mathbb{C}}\left(W_{\mathbb{C}}, W_{\mathbb{C}}^{\prime}\right)$ such that $\operatorname{Re}(T)=(T+\bar{T}) / 2 \in \operatorname{Sym}_{\mathbb{R}}^{+}\left(W_{\infty}, W_{\infty}^{\prime}\right)$. Then $\beta_{W}$ is a $\mathbb{C}^{1}$-valued real analytic 2 -cocycle of order two:

(1) $\beta_{W}(\tau, \delta) \beta_{W}(\sigma \tau, \delta)^{-1} \beta_{W}(\sigma, \tau \delta) \beta_{W}(\sigma, \tau)^{-1}=1$ and $\beta_{E}(1, \sigma)=\beta_{W}(\sigma, 1)=1$,

(2) $\beta_{W}(\sigma, \tau)^{2}=\zeta_{W}(\tau) \zeta_{W}(\sigma \tau)^{-1} \zeta_{W}(\sigma)$ where $\zeta_{W}(\sigma)=\operatorname{det} J\left(\sigma, z_{0}\right) /\left|\operatorname{det} J\left(\sigma, z_{0}\right)\right|$.

Let $M p\left(W_{\infty}\right)=\mathbb{C}^{1} \times G_{\infty}$ be a real Lie group with a multiplication law

$$
(\varepsilon, \sigma) \cdot(\eta, \tau)=\left(\varepsilon \eta \beta_{W}(\sigma, \tau), \sigma \tau\right) .
$$

Then $\widetilde{G}_{\infty}$ is identified with a closed subgroup of the $(\varepsilon, \sigma) \in M p\left(W_{\infty}\right)$ such that $\varepsilon^{2}=\zeta_{W}(\sigma)^{-1}$, and the covering map is $\varpi_{\infty}(\varepsilon, \sigma)=\sigma$ [Tak3].

For $\widetilde{\sigma}=(\varepsilon, \sigma) \in \widetilde{G}_{\infty}$ and $z \in \mathfrak{H}_{W}$, put

$$
J_{\frac{1}{2}}(\widetilde{\sigma}, z)=\varepsilon^{-1} \varepsilon_{W}\left(\sigma ; z, z_{0}\right)|\operatorname{det} J(\sigma, z)|^{1 / 2}
$$

which is real analytic on $\widetilde{\sigma} \in \widetilde{G}_{\infty}$, holomorphic on $z \in \mathfrak{H}_{W}$ and satisfies the relations [Tak3]

$$
J_{\frac{1}{2}}(\widetilde{\sigma} \widetilde{\tau}, z)=J_{\frac{1}{2}}(\widetilde{\sigma}, \tau(z)) J_{\frac{1}{2}}(\widetilde{\tau}, z) \quad \text { and } \quad J_{\frac{1}{2}}(\widetilde{\sigma}, z)^{2}=\operatorname{det} J(\sigma, z) .
$$

Let us denote by $\delta_{0}=\operatorname{det}^{\frac{1}{2}}$ the character $\widetilde{k}=(\varepsilon, k) \mapsto J_{\frac{1}{2}}\left(\widetilde{k}, z_{0}\right)=\varepsilon^{-1}$ of a maximal compact subgroup $\widetilde{K}_{\infty}=\varpi_{\infty}^{-1}\left(K_{\infty}\right)$ of $\widetilde{G}_{\infty}$.

1.4.4. The Siegel upper half space $\mathfrak{H}_{W}$ is identified with a subspace of $\mathfrak{H}_{\mathbb{W}}$ by $\mathfrak{H}_{W} \ni z=[w \mapsto w z] \in \mathfrak{H}_{\mathbb{W}}$. Then the real Lie group $M p\left(\mathbb{W}_{\infty}\right)$ is defined by a 2-cocycle $\beta_{\mathbb{W}}$ subordinate to $z_{0} \in \mathfrak{H}_{W} \hookrightarrow \mathfrak{H}_{\mathbb{W}}$, and the covering group $\widetilde{S p}\left(\mathbb{V}_{\infty}\right)$ is identified with a closed subgroup of $M p\left(\mathbb{W}_{\infty}\right)$. Under these identifications, the group homomorphism $\widetilde{\rho}_{\infty}: \widetilde{G}_{\infty} \rightarrow \widetilde{S p}\left(\mathbb{V}_{\infty}\right)$ is expressed by

$$
\widetilde{\rho}_{\infty}(\varepsilon, \sigma)=\left(\varepsilon^{m}, \rho(\sigma)\right) \text { for all }(\varepsilon, \sigma) \in \widetilde{G}_{\infty} \subset M p\left(W_{\infty}\right) .
$$


Let $H_{z_{0}}$ be a complex Hilbert space consisting of the holomorphic $\mathbb{C}$-valued functions $\varphi$ on $\mathbb{W}_{\mathbb{C}}^{\prime}$ such that

$$
|\varphi|^{2}=\int_{\mathbb{W}_{\mathbb{C}}^{\prime}}|\varphi(w)|^{2} \kappa_{S}\left(z_{0}, w\right) d_{z_{0}}(e)<\infty .
$$

Then the contragredient representation $\check{\omega}_{\infty}$ of the Weil representation $\omega_{\infty}$ of $\widetilde{S p}\left(\mathbb{V}_{\infty}\right)$ is realized on $H_{z_{0}}$ [Tak3]. So the contragredient representation $\check{\omega}_{S, \infty}$ of $\omega_{S, \infty}$ is realized also on $H_{z_{0}}$.

1.4.5. Let us define the holomorphic discrete series of $\widetilde{G}_{\infty}$. Let $\delta_{\mathbb{C}}$ be the irreducible representation of $G L_{\mathbb{C}}\left(W_{\mathbb{C}}^{\prime}\right)$ of the Young diagram

\begin{tabular}{|c|c|c|c|c|c|c|c|}
\hline 1 & 2 & $\cdots$ & & $\cdots$ & & $\cdots$ & $\ell_{1}$ \\
\hline 1 & 2 & $\cdots$ & & $\ldots$ & $\ell_{2}$ & & \\
\hline & & $\cdots$ & & $\cdots$ & & & \\
\hline 1 & 2 & $\cdots$ & $\ell_{n}$ & & & & \\
\hline
\end{tabular}

with $\ell_{1} \geq \ell_{2} \geq \cdots \geq \ell_{n} \geq 0$. Let $V_{\delta}$ be the representation space of $\delta_{\mathbb{C}}$ with a hermitian inner product $\langle,\rangle_{\delta}$ such that

$\left\langle\delta_{\mathbb{C}}(d) v, v^{\prime}\right\rangle_{\delta}=\left\langle v, \delta_{\mathbb{C}}\left(\left(\operatorname{Im} z_{0}\right)^{-1} \cdot{ }^{t} \bar{d} \cdot \operatorname{Im} z_{0}\right) v^{\prime}\right\rangle_{\delta}$ for all $d \in G L_{\mathbb{C}}\left(W_{\mathbb{C}}^{\prime}\right)$ and $v, v^{\prime} \in V_{\delta}$

Then $\delta(k)=\delta_{\mathbb{C}}\left(J\left(k, z_{0}\right)\right)$ is a unitary irreducible representation of $K_{\infty}$. It is also regarded as a representation of $\widetilde{K}_{\infty}$ via the projection onto $K_{\infty}$. Put

$$
\mathcal{K}_{\delta}\left(z^{\prime}, z\right)=\delta_{\mathbb{C}}\left[\left(\frac{z^{\prime}-\bar{z}}{2 \sqrt{-1}}\right)^{-1} \cdot \operatorname{Im} z_{0}\right] \in G L_{\mathbb{C}}\left(V_{\delta}\right) \quad\left(z, z^{\prime} \in \mathfrak{H}_{W}\right) .
$$

Let $H_{\delta \otimes \delta_{0}^{-m}}$ be a complex Hilbert space consisting of the holomorphic $V_{\delta}$-valued functions $\varphi$ on $\mathfrak{H}_{W}$ such that

$$
|\varphi|^{2}=\int_{\mathfrak{H}_{W}} \operatorname{det}(\operatorname{Im} z)^{-m / 2}\left\langle\mathcal{K}_{\delta}(z, z)^{-1} \varphi(z), \varphi(z)\right\rangle_{\delta} d(z)<\infty .
$$

The action $\pi_{\delta \otimes \delta_{0}^{-m}}$ of $\widetilde{G}_{\infty}$ on $H_{\delta \otimes \delta_{0}^{-m}}$ is defined by

$$
\left(\pi_{\delta \otimes \delta_{0}^{-m}}(\widetilde{\sigma}) \varphi\right)(z)=J_{\delta \otimes \delta_{0}^{-m}}\left(\widetilde{\sigma}^{-1}, z\right)^{-1} \varphi\left(\sigma^{-1}(z)\right) \quad \text { for } \widetilde{\sigma} \in \widetilde{G}_{\infty}, \varphi \in H_{\delta \otimes \delta_{0}^{-m}} .
$$

Here we put

$$
J_{\delta \otimes \delta_{0}^{-m}}(\widetilde{\sigma}, z)=\delta_{\mathbb{C}}(J(\sigma, z)) J_{\frac{1}{2}}(\widetilde{\sigma}, z)^{-m} .
$$

If $\ell_{n}-m / 2>n$, then $\left(\pi_{\delta \otimes \delta_{0}^{-m}}, H_{\delta \otimes \delta_{0}^{-m}}\right)$ is the irreducible square-integrable unitary representation, a so-called holomorphic discrete series, of $\widetilde{G}_{\infty}$ of minimal $\widetilde{K}_{\infty}$-type $\delta \otimes \delta_{0}^{-m}=\delta \otimes \operatorname{det}^{-m / 2}$. The square-integrable representation $\pi_{\delta \otimes \delta_{0}^{-m}}$ is integrable if and only if $\ell_{n}-m / 2>2 n$. See Chapter VI, $\S 4$ and Chapter IX, $\S 7$ of [Knp] for the construction and the minimal $K$-type of the holomorphic discrete series of a connected semi-simple real Lie group. It is easy to show that the construction of the holomorphic discrete series given in [Knp] is equivalent to our construction. See $[\mathrm{H}-\mathrm{S}]$ for the integrable discrete series. The arguments of $[\mathrm{Knp}]$ and $[\mathrm{H}-\mathrm{S}]$ can be applied to our non-linear Lie group $\widetilde{G}_{\infty}$. 
1.4.6. Now we will consider the holomorphic discrete series of the Jacobi group $G_{J, \infty}$. Let $H_{\delta, S}$ be a complex Hilbert space consisting of the $V_{\delta}$-valued holomorphic functions $\varphi$ on $\mathfrak{H}_{W, J}$ such that

$$
|\varphi|^{2}=\int_{\mathfrak{H}_{W, J}}\left\langle\mathcal{K}_{\delta}(z, z)^{-1} \varphi(z, w), \varphi(z, w)\right\rangle_{\delta} \kappa_{S}(z, w) d(z, w)<\infty .
$$

The action $\pi_{\delta, S}$ of $G_{J, \infty}$ on $H_{\delta, S}$ is defined by

$$
\left(\pi_{\delta, S}(g) \varphi\right)(Z)=J_{\delta, S}\left(g^{-1}, Z\right)^{-1} \varphi\left(g^{-1}(Z)\right)
$$

for $g \in G_{J, \infty}$ and $\varphi \in H_{\delta, S}$. Here we put

$$
J_{\delta, S}(g, Z)=\eta_{S}(g ; Z)^{-1} J_{\delta}(\sigma, z)
$$

for $g=(\sigma, h) \in G_{J, \infty}$ and $Z=(z, w) \in \mathfrak{H}_{W, J}$.

Then $\left(\pi_{\delta, S}, H_{\delta, S}\right)$ is an irreducible unitary representation (possibly $H_{\delta, S}=\{0\}$ ). Let $\widetilde{\pi}_{\delta, S}$ be the composition of $\pi_{\delta, S}$ with the covering map of $\widetilde{G}_{J, \infty}$ onto $G_{J, \infty}$. Then we proved in [Tak2] that

Theorem. Suppose $\ell_{n}-m / 2>n$.

1) Then $\left(\pi_{\delta \otimes \delta_{0}^{-m}}\right)_{J} \otimes \breve{\omega}_{S, \infty}$ is unitarily equivalent to $\widetilde{\pi}_{\delta, S}$ by the unitary mapping $\varphi \otimes \psi \mapsto \varphi \otimes \psi$ defined by

$$
\begin{aligned}
(\varphi \otimes \psi)\left(g\left(Z_{0}\right)\right)=J_{\delta, S}\left(g ; Z_{0}\right)\left(\pi_{\delta \otimes \delta_{0}^{-m}}(\widetilde{\sigma})^{-1} \varphi\right)\left(z_{0}\right) \\
\cdot\left(\check{\omega}_{S, \infty}(\widetilde{\sigma}, h)^{-1} \psi\right)(0) \cdot\left(\operatorname{det} \operatorname{Im} z_{0}\right)^{-m / 4}
\end{aligned}
$$

for $g=(\sigma, h) \in G_{J, \infty}$ and $Z_{0}=\left(z_{0}, 0\right) \in \mathfrak{H}_{W, J}$.

2) The $\delta$-isotypic component of $\widetilde{\pi}_{\delta, S}$ is the tensor product of the $\delta \otimes \delta_{0}^{-m}$-isotypic component of $\pi_{\delta \otimes \delta_{0}^{-m}}$ and the $\delta_{0}^{m}=\operatorname{det}^{m / 2}$-isotypic component of $\check{\omega}_{S, \infty}$. In particular, the multiplicity of $\delta$ in $\pi_{\delta, S}$ is equal to one.

Remark. The coset space $G_{J, \infty} / K_{\infty} \times \mathbb{U}_{\infty}$ is isomorphic to $\mathfrak{H}_{W, J}$ by $\dot{g} \mapsto g\left(Z_{0}\right)$. Then the induced representation $\operatorname{Ind}\left(G_{J, \infty}, K_{\infty} \times \mathbb{U}_{\infty} ; \delta \otimes \chi_{S, \infty}^{-1}\right)$ is realized on a complex Hilbert space of the locally integrable $V_{\delta}$-valued functions $\varphi$ on $\mathfrak{H}_{W, J}$ such that

$$
|\varphi|^{2}=\int_{\mathfrak{H}_{W, J}}\left\langle\mathcal{K}_{\delta}(z, z)^{-1} \varphi(z, w), \varphi(z, w)\right\rangle_{\delta} \kappa_{S}(z, w) d(z, w)<\infty
$$

with the action

$$
(g \cdot \varphi)(Z)=J_{\delta, S}\left(g^{-1} ; Z\right)^{-1} \varphi\left(g^{-1}(Z)\right) \quad \text { for } g \in G_{J, \infty}, \varphi \in \mathfrak{H}_{W, J} .
$$

In this sense, $\pi_{\delta, S}$ is a holomorphic induction on $G_{J, \infty}$ or a holomorphic discrete series on $G_{J, \infty}$.

1.4.7. The irreducible unitary representation $\omega_{S, \infty}$ of $\widetilde{G}_{J, \infty}$ has the minimal $\widetilde{K}_{\infty}$ type $\delta_{0}^{-m}=\operatorname{det}^{-m / 2}$ with multiplicity one $([\mathrm{K}-\mathrm{V}])$. Let us describe the normal vector in the $\delta_{0}^{-m}$-isotypic component. Put

$$
\vartheta_{S}(Z)=\sum_{\ell \in \mathcal{L}} \mathbf{e}_{\infty}\left(\frac{1}{2}\langle\ell, \ell z\rangle_{S}+\langle\ell, w\rangle_{S}\right) \quad \text { for } Z=(z, w) \in \mathfrak{H}_{W, J}
$$

Then we have a transformation formula

$$
\vartheta_{S}((\gamma, \lambda) Z)=\xi_{S, \infty}(\lambda) \varepsilon_{\Gamma}(\widetilde{\gamma}) \eta_{S}((\gamma, \lambda) ; Z)^{-1} J_{\frac{1}{2}}(\widetilde{\gamma}, z)^{-m} \vartheta_{S}(Z)
$$


for all $(\gamma, \lambda) \in \Gamma_{J}=\Gamma \ltimes \Lambda_{\infty}[$ Tak3, Th.5.2]. Put

$$
\Theta_{S, \infty}(\widetilde{g})=2^{m n / 4} \eta_{S}\left(g ; Z_{0}\right) J_{\frac{1}{2}}\left(\widetilde{\sigma}, z_{0}\right)^{-m} \vartheta_{S}\left(g\left(Z_{0}\right)\right)
$$

for $\widetilde{g}=(\widetilde{\sigma}, h) \in \widetilde{G}_{J, \infty}=\widetilde{G}_{\infty} \ltimes H\left[\mathbb{V}_{\infty}, D\right]$ and $Z_{0}=\left(z_{0}, 0\right) \in \mathfrak{H}_{W, J}$. Here we put $g=(\sigma, h) \in G_{J, \infty}$. Then we have a transformation formula

$$
\Theta_{S, \infty}((\widetilde{\gamma}, \lambda) \widetilde{g})=\xi_{S, \infty}(\lambda) \varepsilon_{\Gamma}(\widetilde{\gamma}) \Theta_{S, \infty}(\widetilde{g}) \quad \text { for all }(\widetilde{\gamma}, \lambda) \in \widetilde{\Gamma}_{J}=\widetilde{\Gamma} \ltimes \Lambda_{\infty} .
$$

If $\omega_{S, \infty}$ is realized on $L^{2}\left(\mathbb{W}_{\infty}\right)$, the $\delta_{0}^{-m}$-isotypic component has a normal base

$$
\psi_{0}(x)=\operatorname{det}\left(2 \operatorname{Im} z_{0}\right)^{m / 4} \mathbf{e}_{\infty}\left(\frac{1}{2}\left\langle x, x z_{0}\right\rangle_{S}\right) \quad\left(x \in \mathbb{W}_{\infty}\right) .
$$

The corresponding vector in $\operatorname{Ind}\left(H\left[\mathbb{W}_{\infty}, D\right], \Lambda_{\infty} ; \xi_{S, \infty}\right)$ is

$$
\Theta_{\psi_{0}}(h)=\sum_{\ell \in \mathcal{L}} \psi_{0}(x+\ell) \mathbf{e}_{\infty}\left(\operatorname{tr}(S \cdot t)+\frac{1}{2}\langle x, y\rangle_{S}+\langle\ell, y\rangle\right)
$$

for $h=((x, y), t) \in H\left[\mathbb{V}_{\infty}, D\right]$. We have

$$
\left(\omega_{S, \infty}(\widetilde{g}) \psi_{0}\right)(x)=\varepsilon^{m} \eta_{S}\left(g ; Z_{0}\right) \operatorname{det}(2 \operatorname{Im} z)^{m / 4} \mathbf{e}_{\infty}\left(\frac{1}{2}\langle x, x z\rangle_{S}+\langle x, w\rangle_{S}\right)
$$

for all $\widetilde{g}=(\widetilde{\sigma}, h) \in \widetilde{G}_{J, \infty}$ with $\widetilde{\sigma}=(\varepsilon, \sigma) \in \widetilde{G}_{\infty}$ and $g\left(Z_{0}\right)=(z, w)$ (see the proof of Th.5.2 in [Tak3]). Then we have a formula

$$
\left(\omega_{S, \infty}(\widetilde{g}) \Theta_{\psi_{0}}\right)(1)=\Theta_{S, \infty}(\widetilde{g}) \quad \text { for all } \widetilde{g} \in \widetilde{G}_{J ; \infty} .
$$

1.4.8. Theorem 1.4.1 and Theorem 1.4.6 imply that the $\check{\pi}_{\delta, S}$-isotypic component in $\operatorname{Ind}\left(G_{J, \infty}, \Gamma_{J} ; \alpha \otimes \xi_{S, \infty}\right)$ corresponds to the $\check{\pi}_{\delta \otimes \delta_{0}^{-m}}$-isotypic component in $\operatorname{Ind}\left(\widetilde{G}_{\infty}, \widetilde{\Gamma} ; \widetilde{\alpha} \otimes \varepsilon_{\Gamma}^{-1}\right)$. Such a correspondence induces a correspondence between the spaces of automorphic forms on $G_{J, \infty}$ and $\widetilde{G}_{\infty}$ via the isometry of Appendix A.3. Let us now describe explicitly the correspondence.

Let us denote by $\mathcal{A}\left(\Gamma_{J}, \alpha ; \delta, S\right)$ the space of the automorphic forms on $G_{J, \infty}$ associated with the data

$$
(G, K, \Gamma, E ; \xi, \pi, \delta)=\left(G_{J, \infty}, K_{\infty}, \Gamma_{J}, \mathbb{U}_{\infty} ;\left(\alpha \otimes \xi_{S, \infty}\right)^{-1}, \pi_{\delta, S}, \delta\right)
$$

in the sense of Appendix A.2. Let us also denote by $\mathcal{A}\left(\widetilde{\Gamma}, \widetilde{\alpha} ; \delta \otimes \operatorname{det}^{-m / 2}\right)$ the space of the automorphic forms on $\widetilde{G}_{\infty}$ associated with the data

$$
(G, K, \Gamma, E ; \xi, \pi, \delta)=\left(\widetilde{G}_{\infty}, \widetilde{K}_{\infty}, \widetilde{\Gamma}, E_{\infty} ; \widetilde{\alpha}^{-1} \otimes \varepsilon_{\Gamma}, \pi_{\delta \otimes \delta_{0}^{-m}}, \delta \otimes \delta_{0}^{-m}\right) .
$$

Recall that the multiplicity of $\delta$ (resp. $\left.\delta \otimes \operatorname{det}^{-m / 2}\right)$ in $\left.\pi_{\delta, S}\right|_{K_{\infty}}\left(\right.$ resp. $\left.\pi_{\delta \otimes \delta_{0}^{-m}}\right|_{\widetilde{K}_{\infty}}$ ) is equal to one. Then we have

Theorem. $A \mathbb{C}$-linear isometry $F \mapsto f_{F}$ from $\mathcal{A}\left(\Gamma_{J}, \alpha ; \delta, S\right)$ onto $\mathcal{A}(\widetilde{\Gamma}, \widetilde{\alpha} ; \delta \otimes$ $\operatorname{det}^{-m / 2}$ ) is defined by

$$
f_{F}(\widetilde{\sigma})=\int_{\Lambda_{\infty} \backslash H\left[\mathbb{V}_{\infty}, D\right]} F\left(\sigma, h^{\sigma}\right) \overline{\Theta_{S, \infty}\left(\widetilde{\sigma}, h^{\sigma}\right)} d(\dot{h}) .
$$

Proof. We have $\tilde{\pi}_{\delta, S}=\pi_{\delta \otimes \delta_{0}^{-m}} \otimes \check{\omega}_{S, \infty}$ by Theorem 1.4.6. It is proved in [Tak2] that the irreducible unitary representation $\pi_{\delta, S}$ of $G_{J, \infty}$ has the minimal $K_{\infty}$-type $\delta$ with multiplicity one which is the tensor product of the $\delta \otimes \delta_{0}^{-m}$-isotypic component of 
$\pi_{\delta \otimes \delta_{0}^{-m}}$ and the $\delta_{0}^{m}$-isotypic component of $\check{\omega}_{S, \infty}$. For any $\varphi \in \operatorname{Ind}\left(\widetilde{G}_{\infty}, \widetilde{\Gamma} ; \widetilde{\alpha} \otimes \varepsilon_{\Gamma}^{-1}\right)$ and $\psi \in \operatorname{Ind}\left(H\left[\mathbb{V}_{\infty}, D\right], \Lambda_{\infty} ; \xi_{S, \infty}\right)$ such that $|\psi|=1$, we have

$$
\varphi(\widetilde{\sigma})=\int_{\Lambda_{\infty} \backslash H\left[\mathbb{V}_{\infty}, D\right]}(\varphi \otimes \psi)\left(\sigma, h^{\sigma}\right)\left(\overline{\left.\omega_{S, \infty}\left(\widetilde{\sigma}, h^{\sigma}\right) \psi\right)(1)} d(\dot{h})\right.
$$

for all $\widetilde{\sigma} \in \widetilde{G}_{\infty}$. Then we have a $\mathbb{C}$-linear isometry

$$
T: \operatorname{Ind}\left(G_{J, \infty}, \Gamma_{J} ; \alpha \otimes \xi_{S, \infty} ; \check{\pi}_{\delta, S} ; \check{\delta}\right) \stackrel{\sim}{\rightarrow} \operatorname{Ind}\left(\widetilde{G}_{\infty}, \widetilde{\Gamma} ; \widetilde{\alpha} \otimes \varepsilon_{\Gamma}^{-1} ; \check{\pi}_{\delta \otimes \delta_{0}^{-m}} ; \check{\delta} \otimes \delta_{0}^{m}\right)
$$

defined by

$$
(T \varphi)(\widetilde{\sigma})=\int_{\Lambda_{\infty} \backslash H\left[\mathbb{V}_{\infty}, D\right]} \varphi\left(\sigma, h^{\sigma}\right) \overline{\Theta_{S, \infty}\left(\widetilde{\sigma}, h^{\sigma}\right)} d(\dot{h}), \quad\left(\widetilde{\sigma} \in \widetilde{G}_{\infty}\right)
$$

for all $\varphi \in \operatorname{Ind}\left(G_{J, \infty}, \Gamma_{J} ; \alpha \otimes \xi_{S, \infty} ; \check{\pi}_{\delta, S} ; \check{\delta}\right)$. Then the isometry of Appendix A.3 gives the required isometry.

Remark. In $\S 3.2$, we will show that the correspondence $F \mapsto f_{F}$ is compatible with the action of the Hecke operators.

1.4.9. We will give a variation of Theorem 1.4.8. For any $F \in \mathcal{A}\left(\Gamma_{J}, \alpha ; \delta, S\right)$, put

$$
F(Z)=J_{\delta, S}\left(g, Z_{0}\right) F(g) \text { for } Z=g\left(Z_{0}\right) \in \mathfrak{H}_{W, J} \text { with } g \in G_{J, \infty},
$$

which is a well-defined $V_{\delta}$-valued function on $\mathfrak{H}_{W, J}$.

For any $f \in \mathcal{A}\left(\widetilde{\Gamma}, \widetilde{\alpha} ; \delta \otimes \operatorname{det}^{-m / 2}\right)$, put

$$
f(z)=J_{\delta \otimes \delta_{0}^{-m}}\left(\widetilde{\sigma}, z_{0}\right) f(\widetilde{\sigma}) \quad \text { for } z=\sigma\left(z_{0}\right) \in \mathfrak{H}_{W} \text { with } \widetilde{\sigma} \in \widetilde{G}_{\infty},
$$

which is a well-defined $V_{\delta}$-valued function on $\mathfrak{H}_{W}$. Then we have

Theorem. For any $F \in \mathcal{A}\left(\Gamma_{J}, \alpha ; \delta, S\right)$, we have

$$
f_{F}(z)=2^{m n / 4} \operatorname{det}(\operatorname{Im} z)^{m / 2} \int_{L_{z} \backslash \mathbb{W}_{\mathbb{C}}^{\prime}} F(z, w) \overline{\vartheta_{S}(z, w)} \kappa_{S}(z, w) d_{z}(w)
$$

for all $z \in \mathfrak{H}_{W}$. Here $L_{z}=\left\{x z+y \mid x \in \mathcal{L}, y \in \mathcal{L}^{\prime}\right\}$ is a $\mathbb{Z}$-lattice in $\mathbb{W}_{\mathbb{C}}^{\prime}$.

Proof. Using the identity

$$
\left|\eta_{S}\left(g ; Z_{0}\right)\right|^{2}=\kappa_{S}\left(g\left(Z_{0}\right)\right) \quad \text { for all } g \in G_{J, \infty}
$$

and (1.4.2.1), we have

$$
\begin{aligned}
f_{F}(\widetilde{\sigma})= & \int_{\Lambda_{\infty} \backslash H\left[\mathbb{V}_{\infty}, D\right]} F\left(\sigma, h^{\sigma}\right) \overline{\Theta_{S, \infty}\left(\widetilde{\sigma}, h^{\sigma}\right)} d(\dot{h}) \\
= & 2^{m n / 4} J_{\delta}\left(\sigma, z_{0}\right)^{-1} \overline{J_{\frac{1}{2}}\left(\widetilde{\sigma}, z_{0}\right)^{-m}} \\
& \cdot \int_{\Lambda_{\infty} \backslash H\left[\mathbb{V}_{\infty}, D\right]} F(h(z, 0)) \overline{\vartheta_{S}(h(z, 0))} \kappa_{S}(h(z, 0)) d(\dot{h}) \\
= & 2^{m n / 4} \operatorname{det}(\operatorname{Im} z)^{m / 2} J_{\delta \otimes \delta_{0}^{-m}}\left(\widetilde{\sigma}, z_{0}\right)^{-1} \int_{L_{z} \backslash \mathbb{W}_{\mathbb{C}}^{\prime}} F(z, w) \overline{\vartheta_{S}(z, w)} \kappa_{S}(z, w) d_{z}(w) .
\end{aligned}
$$

Here we put $z=\sigma\left(z_{0}\right) \in \mathfrak{H}_{W}$. Then we have the required formula. 
1.4.10. We will consider a classical case. Let us suppose that $\ell_{1}=\ell_{2}=\cdots=\ell_{n}=$ $\ell$. We shall also suppose that $\Gamma$ is commensurable with

$$
\Gamma(1)=\left\{\sigma \in S p(V) \mid\left(L \oplus L^{\prime}\right) \sigma=L \oplus L^{\prime}\right\} .
$$

Let $S_{\ell-\frac{m}{2}}\left(\widetilde{\Gamma}, \widetilde{\alpha} \otimes \varepsilon_{\Gamma}^{-1}\right)$ be the space of Siegel cusp forms of weight $\ell-\frac{m}{2}$ with respect to $\widetilde{\Gamma}$ with character $\widetilde{\alpha} \otimes \varepsilon_{\Gamma}^{-1}$, that is, the $\mathbb{C}$-valued holomorphic functions $f$ on $\mathfrak{H}_{W}$ such that

1) $f(\gamma(z))=\widetilde{\alpha} \otimes \varepsilon_{\Gamma}^{-1}(\widetilde{\gamma}) \cdot J_{\frac{1}{2}}(\widetilde{\gamma}, z)^{2 \ell-m} f(z)$ for all $\widetilde{\gamma} \in \widetilde{\Gamma}$,

2) $|f(z)| \operatorname{det}(\operatorname{Im} z)^{(2 \ell-m) / 4}$ is bounded on $\mathfrak{H}_{W}$.

Let $J_{\ell, S}^{\text {cusp }}\left(\Gamma_{J}, \alpha\right)$ be the space of the cuspidal Jacobi forms of weight $\ell$ and index $S$ with respect to $\Gamma_{J}$ with character $\alpha$, that is, the holomorphic $\mathbb{C}$-valued functions $F$ on $\mathfrak{H}_{W, J}$ such that

(1) $F(\gamma(Z))=\alpha \otimes \xi_{S, \infty}(\gamma) J_{\delta, S}(\gamma, Z) F(Z)$ for all $\gamma \in \Gamma_{J}$,

(2) $|F(z, w)| \operatorname{det}(\operatorname{Im} z)^{\ell / 2} \exp \left(\pi\left\langle\operatorname{Im} w(\operatorname{Im} z)^{-1}, \operatorname{Im} w\right\rangle_{S}\right)$ is bounded on $\mathfrak{H}_{W, J}$.

The condition (1) is equivalent to the following conditions:

(i) $F\left(\gamma(z), w(c z+d)^{-1}\right)=\alpha(\gamma) \operatorname{det}(c z+d)^{\ell} \exp \left(\pi \sqrt{-1}\left\langle w^{t} c, w(c z+d)^{-1}\right\rangle_{S}\right)$. $F(z, w)$ for all $\gamma=\left[\begin{array}{ll}a & b \\ c & d\end{array}\right] \in \Gamma$,

(ii) $F(z, w+x z+y)=\exp 2 \pi \sqrt{-1}\left(-\frac{1}{2}\langle x, x z\rangle_{S}-\langle x, w\rangle_{S}\right) \cdot F(z, w)$ for all $x \in \mathcal{L}$ and $y \in \mathcal{L}^{\prime}$.

The condition (2) is equivalent to the following condition:

(iii) for any $\gamma \in \Gamma(1) \ltimes \Lambda_{\infty}$, the conjugate $F^{\gamma}(Z)=J_{\delta, S}(\gamma, Z)^{-1} F(\gamma(Z))$ has the Fourier expansion

$$
F^{\gamma}(z, w)=\sum_{x, c} a(x, c) \mathbf{e}_{\infty}\left(\operatorname{tr}\left(M^{-1} c z\right)+\langle x, w\rangle_{S}\right)
$$

with a suitable $0<M \in \mathbb{Z}$. Here $\sum_{x, c}$ is the summation over the $x \in \mathcal{L}$ and $c \in \operatorname{Sym}_{\mathbb{Z}}^{*}\left(\mathcal{L}^{\prime}, \mathcal{L}\right)$ such that $M^{-1} c-\frac{1}{2}^{t} x x$ is positive definite.

The notation in the condition (iii) is defined as follows:

$$
\begin{aligned}
& \operatorname{Sym}_{\mathbb{Z}}\left(\mathcal{L}, \mathcal{L}^{\prime}\right)=\left\{b \in \operatorname{Sym}_{\mathbb{Q}}\left(\mathbb{W}, \mathbb{W}^{\prime}\right) \mid \mathcal{L} b \subset \mathcal{L}^{\prime}\right\}, \\
& \operatorname{Sym}_{\mathbb{Z}}^{*}\left(\mathcal{L}^{\prime}, \mathcal{L}\right)=\left\{c \in \operatorname{Sym}_{\mathbb{Q}}\left(\mathbb{W}^{\prime}, \mathbb{W}\right) \mid \operatorname{tr}(b c) \in \mathbb{Z} \text { for all } b \in \operatorname{Sym}_{\mathbb{Z}}\left(\mathcal{L}, \mathcal{L}^{\prime}\right)\right\} .
\end{aligned}
$$

For any $x \in \mathbb{W}$, define a ${ }^{t} x x \in \operatorname{Sym}_{\mathbb{Q}}\left(\mathbb{W}^{\prime}, \mathbb{W}\right)$ such that $\operatorname{tr}\left({ }^{t} x x \cdot b\right)=\langle x, x b\rangle_{S}$ for all $b \in \operatorname{Sym}_{\mathbb{Q}}\left(\mathbb{W}, \mathbb{W}^{\prime}\right)$.

If $\ell-m / 2>2 n$, we have an identification

$$
\mathcal{A}\left(\widetilde{\Gamma}, \widetilde{\alpha} ; \delta \otimes \operatorname{det}^{-m / 2}\right)=S_{\ell-\frac{m}{2}}\left(\widetilde{\Gamma}, \widetilde{\alpha} \otimes \varepsilon_{\Gamma}^{-1}\right)
$$

by $f(\widetilde{\sigma}) \mapsto f(z)$ in the notation of $\mathbf{1 . 4 . 9}$ [Tak3, Th.7.2].

If $\ell-m / 2>2 n+m / 2$, we have an identification

$$
\mathcal{A}\left(\Gamma_{J}, \alpha ; \delta, S\right)=J_{\ell, S}^{\text {cusp }}\left(\Gamma_{J}, \alpha\right)
$$

by $F(g) \mapsto F(Z)$ in the notation of $\mathbf{1 . 4 . 9}$ [Tak1, Th.10.3].

Now Theorem 1.4.9 implies the following 
Theorem. Suppose that $\ell>2 n+m$. Then a $\mathbb{C}$-linear isometry $F \mapsto f_{F}$ from $J_{\ell, S}^{\text {cusp }}\left(\Gamma_{J}, \alpha\right)$ onto $S_{\ell-\frac{m}{2}}\left(\widetilde{\Gamma}, \widetilde{\alpha} \otimes \varepsilon_{\Gamma}^{-1}\right)$ is defined by

$$
f_{F}(z)=2^{m n / 4} \operatorname{det}(\operatorname{Im} z)^{m / 2} \int_{L_{z} \backslash \mathbb{W}_{\mathbb{C}}^{\prime}} F(z, w) \overline{\vartheta_{S}(z, w)} \kappa_{S}(z, w) d_{z}(w) \quad\left(z \in \mathfrak{H}_{W}\right)
$$

1.4.11. Let $\Gamma_{0}$ be a subgroup of $S p(V)$ consisting of the $\gamma=\left[\begin{array}{ll}a & b \\ c & d\end{array}\right] \in S p(V)$ such that

(1) $\left(L \oplus L^{\prime}\right) \gamma=L \oplus L^{\prime}$,

(2) $\left[v a+v^{\prime} c, v b+v^{\prime} d\right] \equiv\left[v, v^{\prime}\right](\bmod 2 \mathbb{Z})$ for all $v \in L$ and $v^{\prime} \in L^{\prime}$.

Let $M^{*}$ be the dual lattice of $M$ in $U$ (see 1.1.3). Then we have

Proposition. If $M^{*} S \subset M$, then $\rho\left(\Gamma_{0}\right) \subset S p(\mathcal{L})$.

\section{Chapter 2. Finite Local Theory}

Through out this chapter, we will fix a finite place $p$ of $\mathbb{Q}$ such that

1) $\left\{u_{1}, \cdots, u_{m}\right\}$ is a $\mathbb{Z}_{p}$-basis of $M_{p}$,

2) $p \neq 2$ and $M_{p}^{*}=M_{p}$ (see 1.1.5).

\subsection{Algebras of Hecke operators.}

2.1.1. Put $H\left[\mathcal{L}_{p}, D\right]=\left(\mathcal{L}_{p} \oplus \mathcal{L}_{p}^{\prime}\right) \times \mathcal{M}_{p}$ which is an open compact subgroup of $H\left[\mathbb{V}_{p}, D\right]$. The compact group

$$
K_{p}=S p\left(L_{p}\right)=\left\{\sigma \in S p\left(V_{p}\right) \mid\left(L \oplus L^{*}\right) \sigma=L \oplus L^{*}\right\}
$$

acts on $H\left[\mathcal{L}_{p}, D\right]$. Then the semi-direct product $K_{J, p}=K_{p} \ltimes H\left[\mathcal{L}_{p}, D\right]$ is an open compact subgroup of $G_{J, p}$. Put $\mathcal{H}_{J, p}=C_{c}\left(G_{J, p} / / K_{J, p}, \mathbb{U}_{p} ; \chi_{S, p}\right)$ with the notation of Appendix B.2.

$\widetilde{K}_{p}=\widetilde{S p}\left(L_{p}\right)$ is an open compact subgroup of $\widetilde{G}_{p}$ (see 1.2.4 for the definition of $\left.\widetilde{S p}\left(L_{p}\right)\right)$. Then put $\mathcal{H}_{p}=C_{c}\left(\widetilde{G}_{p} / / \widetilde{K}_{p}, E_{p} ; \nu_{p}^{m}\right)$ with the notation of Appendix B.2. Here $E_{p}$ is the kernel of the covering mapping $\varpi_{p}: \widetilde{G}_{p} \rightarrow G_{p}$, and $\nu_{p}$ is the unique non-trivial character of $E_{p}$.

Proposition. $\widetilde{\rho}_{p}\left(\widetilde{K}_{p}\right) \subset \widetilde{S p}\left(\mathcal{L}_{p}\right)$. More precisely, $\widetilde{\rho}_{p}\left(\mathbf{r}_{L_{p}}^{\prime}(\gamma)\right)=\mathbf{r}_{\mathcal{L}_{p}}^{\prime}(\rho(\gamma))$ for all $\gamma \in S p\left(L_{p}\right)$.

Proof. Because $\left\{u_{1}, \cdots, u_{m}\right\}$ is a $\mathbb{Z}_{p}$-basis of $M_{p}$, we can identify $\mathcal{L}_{p}$ with $\bigoplus^{m} L_{p}$ by $\ell=\left(u_{j} \ell\right)_{j=1, \cdots, m}$. Then we have

$$
\Theta_{\varphi}(h)=\prod_{j=1}^{m} \Theta_{\varphi_{j}}\left(\left(u_{j} x, u_{j} y\right), t / m\right)
$$

for any $\varphi=\bigotimes_{j=1}^{m} \varphi_{j} \in L^{2}\left(\mathbb{W}_{p}\right)=\widehat{\otimes} L^{2}\left(W_{p}\right)$ and $h=((x, y), t) \in H_{S}\left[\mathbb{V}_{p}\right]=$ $\mathbb{V}_{p} \times \mathbb{Q}_{p}$. Here we used the formula (1.1.2.1). Then we have

$$
\mathbf{r}_{L_{p}}(\gamma)^{\otimes m}=\mathbf{r}_{\mathcal{L}_{p}}(\rho(\gamma)) \quad \text { for all } \gamma \in S p\left(L_{p}\right) .
$$


2.1.2. The Haar measure $d_{G_{p}}(\sigma)$ (resp. $d_{\mathbb{U}_{p}}(s)$ ) is normalized so that $\operatorname{vol}\left(K_{p}\right)=1$ $\left(\operatorname{resp} . \operatorname{vol}\left(\mathcal{M}_{p}\right)=1\right)$. The Haar measure $d_{\widetilde{G}_{p}}(\sigma)$ is normalized so that $\operatorname{vol}\left(\widetilde{K}_{p}\right)=1$. Let $d_{\widetilde{G}_{p} / E_{p}}(\dot{\sigma})$ be the Haar measure on $\widetilde{G}_{P} / E_{p}$ induced from the Haar measure $d_{G_{p}}(\sigma)$ on $G_{p}$ via the canonical isomorphism $\widetilde{G}_{p} / E_{p} \stackrel{\sim}{\rightarrow} G_{p}$. Let $d_{E_{p}}(a)$ be the counting measure on $E_{p}$. Then we have an integral formula

$$
\int_{\widetilde{G}_{p}} \varphi(\sigma) d_{\widetilde{G}_{p}}(\sigma)=\int_{\widetilde{G}_{p} / E_{p}}\left(\int_{E_{p}} \varphi(\sigma a) d_{E_{p}}(a)\right) d_{\widetilde{G}_{p} / E_{p}}(\dot{\sigma}) \text { for all } \varphi \in C_{c}\left(\widetilde{G}_{p}\right) .
$$

2.1.3. With respect to the $\mathbb{Q}_{p}$ basis $\left\{v_{1}, \cdots, v_{n}, v_{1}^{*}, \cdots, v_{n}^{*}\right\}$ of $V_{p}$, we will identify $G_{p}=S p\left(V_{p}\right)$ with a matrix group $S p\left(n, \mathbb{Q}_{p}\right)$. Then $K_{p}$ is identified with $S p\left(n, \mathbb{Z}_{p}\right)$. For an $n$-tuple $\alpha=\left(\alpha_{1}, \cdots, \alpha_{n}\right) \in \mathbb{Z}^{n}$ of integers, put $\mathrm{d}\left(p^{\alpha}\right)=\left[\begin{array}{cc}p^{\alpha} & 0 \\ 0 & p^{-\alpha}\end{array}\right] \in G_{p}$ with $p^{\alpha}=\left[\begin{array}{ccc}p^{\alpha_{1}} & & \\ & \ddots & \\ & & p^{\alpha_{n}}\end{array}\right] \in G L_{n}\left(\mathbb{Q}_{p}\right)$. Then we have a double coset decomposition $G_{p}=\bigsqcup_{\alpha \in \Upsilon} K_{p} \mathrm{~d}\left(p^{\alpha}\right) K_{p}$ where

$$
\Upsilon=\left\{\left(\alpha_{1}, \cdots, \alpha_{n}\right) \in \mathbb{Z}^{n} \mid \alpha_{1} \geq \cdots \geq \alpha_{n} \geq 0\right\} .
$$

Proposition. The support of any $\varphi \in \mathcal{H}_{J, p}$ is contained in $\bigsqcup_{\alpha \in \Upsilon} K_{J, p} \mathrm{~d}\left(p^{\alpha}\right) K_{J, p} \mathbb{U}_{p}$.

Proof. Suppose that $\varphi(g) \neq 0$ for $g=\left(\mathrm{d}\left(p^{\alpha}\right), h\right) \in G_{J, p}$ with $h=((x, y), 0) \in$ $H\left[\mathbb{V}_{p}, D\right]$ and $\alpha \in \Upsilon$. Then for any $\ell \in \mathcal{L}_{p}^{\prime}$, we have

$$
\begin{aligned}
\varphi(g) & =\varphi(g \cdot(1,(0, \ell), 0)) \\
& =\varphi\left(\left(1,\left(0, \ell p^{\alpha}\right), 0\right)\left(\mathrm{d}\left(p^{\alpha}\right),(x, y), D(x, \ell)\right)\right) \\
& =\mathbf{e}_{p}\left(\langle x, \ell\rangle_{S}\right)^{-1} \varphi(g) .
\end{aligned}
$$

This means that $\left\langle x, \mathcal{L}_{p}^{\prime}\right\rangle_{S} \subset \mathbb{Z}_{p}$ and then $x \in \mathcal{L}_{p}$. Similarly for any $\ell \in \mathcal{L}_{p}$, we have

$$
\begin{aligned}
\varphi(g) & =\varphi((1,(\ell, 0), 0) \cdot g) \\
& =\varphi\left(\left(\mathrm{d}\left(p^{\alpha}\right),(x, y) D\left(\ell p^{\alpha}, y\right)\right)\left(1,\left(\ell p^{\alpha}, 0\right), 0\right)\right) \\
& =\mathbf{e}_{p}\left(\left\langle\ell p^{\alpha}, y\right\rangle_{S}\right)^{-1} \varphi(g) .
\end{aligned}
$$

Then $\left\langle\mathcal{L}_{p}, y p^{\alpha}\right\rangle_{S} \subset \mathbb{Z}_{p}$ and $y p^{\alpha} \in \mathcal{L}_{p}^{\prime}$. Now we have

$$
g=\left(1,\left(0, y p^{\alpha}\right), 0\right) \cdot \mathrm{d}\left(p^{\alpha}\right) \cdot\left(1,(x, 0), \frac{1}{2} D(x, y)\right) \in K_{J, p} \cdot \mathrm{d}\left(p^{\alpha}\right) \cdot K_{J, p} \mathbb{U}_{p} .
$$

2.1.4. For any $\alpha \in \Upsilon$, let $\varphi_{\alpha}$ be the characteristic function of $K_{J, p} \mathrm{~d}\left(p^{\alpha}\right) K_{J, p}$ in $G_{J, p}$. Then $\varphi_{\alpha}$ is an element of $C_{c}\left(G_{J, p} / / K_{J, p}\right)$. Put $\varphi_{\alpha, S}=\theta_{\chi_{S, p}}\left(\varphi_{\alpha}\right) \in \mathcal{H}_{J, p}$ in the notation of Appendix B.2. Then

$$
\varphi_{\alpha, S}(g)=\left\{\begin{array}{ll}
\chi_{S, p}(t)^{-1} & \text { if } g \in K_{J, p} \mathrm{~d}\left(p^{\alpha}\right) K_{J, p} \mathbb{U}_{p} \\
0 & \text { otherwise }
\end{array} \text { for } g=(\sigma,(x, y), t) \in G_{J, p} .\right.
$$

By Proposition 2.1.3, $\left\{\varphi_{\alpha, S} \mid \alpha \in \Upsilon\right\}$ is a $\mathbb{C}$-basis of $\mathcal{H}_{J, p}$.

Proposition. $\mathbb{C}$-algebra $\mathcal{H}_{J, p}$ is commutative. 
Proof. Put $\varepsilon=\left[\begin{array}{cc}0 & 1_{n} \\ 1_{n} & 0\end{array}\right] \in G S p\left(n, \mathbb{Q}_{p}\right)$ and $g^{\prime}=\varepsilon g^{-1} \varepsilon^{-1} \in G_{J, p}$ for all $g \in$ $G_{J, p}$. Then $g \mapsto g^{\prime}$ is an anti-group isomorphism of $G_{J, p}$ such that $d_{G_{J, p}}\left(g^{\prime}\right)=$ $d_{G_{J, p}}(g)$ and $t^{\prime}=t$ for all $t \in \mathbb{U}_{p}$. We have $\varphi\left(g^{\prime}\right)=\varphi(g)$ for all $\varphi \in \mathcal{H}_{J, p}$ because $\varphi_{\alpha, S}\left(g^{\prime}\right)=\varphi_{\alpha, S}(g)$ for all $\alpha \in \Upsilon$. Then a simple calculation shows that $\mathcal{H}_{J, p}$ is commutative.

2.1.5. For any $a \in G L_{\mathbb{Q}_{p}}\left(W_{p}\right)$, define an operator $\mathbf{d}_{0}(a) \in \operatorname{Aut}\left(L^{2}\left(W_{p}\right)\right)$ by

$$
(\mathbf{d}(a) \varphi)(w)=|\operatorname{det} a|_{p}{ }^{1 / 2} \varphi(w a) \quad\left(\varphi \in L^{2}\left(W_{p}\right), w \in W_{p}\right) .
$$

Then $\mathbf{d}(a)=\left(\left[\begin{array}{cc}a & 0 \\ 0 & t^{-1}\end{array}\right], \mathbf{d}_{0}(a)\right) \in M p\left(V_{p}\right)$ such that $\Psi_{p}(\mathbf{d}(a))=(\operatorname{det} a,-1)_{p}$ with the Hilbert symbol $(*, *)_{p}$. Put $\eta_{p}=\gamma_{p}(1) \gamma_{p}(-p)$ with the Weil number $\gamma_{p}$. Then we have $\eta_{p}^{2}=(p,-1)_{p}$. Put

$$
\widetilde{\mathrm{d}}\left(p^{\alpha}\right)=\left(\mathrm{d}\left(p^{\alpha}\right), \eta_{p}^{-|\alpha|} \mathbf{d}_{0}\left(p^{\alpha}\right)\right) \in \widetilde{G}_{p}
$$

for $\alpha=\left(\alpha_{1}, \cdots, \alpha_{n}\right) \in \mathbb{Z}^{n}$ with $|\alpha|=\alpha_{1}+\cdots+\alpha_{n}$. Then we have a double coset decomposition $\widetilde{G}_{p}=\bigsqcup_{\alpha \in \Upsilon} \widetilde{K}_{p} \widetilde{\mathrm{d}}\left(p^{\alpha}\right) \widetilde{K}_{p} E_{p}$.

For any $\alpha \in \Upsilon$, let $\psi_{\alpha}$ be the characteristic function of $\widetilde{K}_{p} \widetilde{\mathrm{d}}\left(p^{\alpha}\right) \widetilde{K}_{p}$ in $\widetilde{G}_{p}$. Then $\psi_{\alpha}$ is an element of $C_{c}\left(\widetilde{G}_{p} / / \widetilde{K}_{p}\right)$. Put $\psi_{\alpha, \nu}=\theta_{\nu_{p}^{m}}\left(\psi_{\alpha}\right) \in \mathcal{H}_{p}=C_{c}\left(\widetilde{G}_{p} / / \widetilde{K}_{p}, E_{p} ; \nu_{p}^{m}\right)$. Then $\left\{\psi_{\alpha, \nu} \mid \alpha \in \Upsilon\right\}$ is a $\mathbb{C}$-basis of $\mathcal{H}_{p}$.

\subsection{Zonal spherical function associated with Weil representation.}

2.2.1. The representation $\omega_{S, p}$ of $\widetilde{G}_{J, p}$ on $L^{2}\left(\mathbb{W}_{p}\right)$ is an irreducible unitary representation such that $\left.\omega_{S, p}\right|_{E_{p} \times \mathbb{U}_{p}}=\nu_{p}^{m} \otimes \chi_{S, p}$. The $\widetilde{K}_{J, p}$-invariant vectors of $L^{2}\left(\mathbb{W}_{p}\right)$ are the constant multiples of $\varphi_{\mathcal{L}_{p}}$, the characteristic function of $\mathcal{L}_{p}$ in $\mathbb{W}_{p}$. Then we have the zonal spherical function $\Phi_{S, p}(g)=\left(\omega_{S, p}(g) \varphi_{\mathcal{L}_{p}}, \varphi_{\mathcal{L}_{p}}\right)\left(g \in \widetilde{G}_{J, p}\right)$ which is an element of $\Omega^{+}\left(\widetilde{G}_{J, p} / / \widetilde{K}_{J, p}, E_{p} \times \mathbb{U}_{p} ; \nu_{p}^{m} \otimes \chi_{S, p}\right)$. We have

1) $\Phi_{S, p}\left(\widetilde{\mathrm{d}}\left(p^{\alpha}\right)\right)=\left(\eta_{p}^{-|\alpha|} p^{-|\alpha| / 2}\right)^{m}$,

2) $\operatorname{supp}\left(\Phi_{S, p}\right)=\bigsqcup_{\alpha \in \Upsilon} \widetilde{K}_{J, p} \widetilde{\mathrm{d}}\left(p^{\alpha}\right) \widetilde{K}_{J, p} \cdot\left(E_{p} \times \mathbb{U}_{p}\right)$.

The first equation is due to the fact that $\varphi_{\mathcal{L}_{p}}$ is equal to the $m$-fold tensor product of the characteristic function of $L_{p}$ in $W_{p}$ under the canonical identification $L^{2}\left(\mathbb{W}_{p}\right)=$ $\widehat{\otimes}^{m} L^{2}\left(W_{p}\right)$ given in 1.3.2. The second is proved by the same argument as that used in the proof of Proposition 2.1.3, and by the first equation.

The following result is due to Shintani [Shn].

Proposition. For any $\varphi \in \mathcal{H}_{p}$, let $\varphi_{J}$ be a continuous function on $G_{J, p}$ defined by

$$
\varphi_{J}(\sigma, h)=\varphi(\widetilde{\sigma}) \overline{\Phi_{S, p}(\widetilde{\sigma}, h)} \quad \text { for }(\widetilde{\sigma}, h) \in \widetilde{G}_{J, p} \text { such that } \varpi(\widetilde{\sigma})=\sigma .
$$

Then $\varphi \mapsto \varphi_{J}$ gives a $\mathbb{C}$-algebra isomorphism of $\mathcal{H}_{p}$ onto $\mathcal{H}_{J, p}$.

Proof. We have only to prove that $\varphi \mapsto \varphi_{J}$ is a $\mathbb{C}$-algebra homomorphism. The remark on the support of $\Phi_{S, p}$ given above shows that the mapping is bijective. Recall that $\Pi_{S, p}$ is an irreducible unitary representation of $H\left[\mathbb{V}_{p}, D\right]$ which is integrable modulo $\mathbb{U}_{p}$ of formal degree one. Then for all $\varphi, \psi \in \mathcal{H}_{p}$, we have 


$$
\begin{aligned}
& \left(\varphi_{J} * \psi_{J}\right)(\sigma, h)=\int_{\widetilde{G}_{J, p} / E_{p} \times \mathbb{U}_{p}} \varphi(\widetilde{\tau}) \overline{\Phi_{S, p}\left(\widetilde{\tau}, h^{\prime}\right)} \psi(\widetilde{\tau} \widetilde{\sigma}) \overline{\Phi_{S, p}\left(\left(\widetilde{\tau}, h^{\prime}\right)^{-1}(\widetilde{\sigma}, h)\right)} d\left(\widetilde{\tau}, h^{\prime}\right) \\
& =\int_{\widetilde{G}_{J, p} / E_{p} \times \mathbb{U}_{p}} \varphi(\widetilde{\tau}) \psi\left(\widetilde{\tau}^{-1} \widetilde{\sigma}\right) \overline{\left(\omega_{S, p}(\widetilde{\tau}) \circ \Pi_{S, p}\left(h^{\prime}\right) \varphi_{\mathcal{L}_{p}}, \varphi_{\mathcal{L}_{p}}\right)} \\
& \times\left(\omega_{S, p} \circ \Pi_{S, p}\left(h^{\prime}\right) \varphi_{\mathcal{L}_{p}}, \omega_{S, p}(\widetilde{\sigma}, h) \varphi_{\mathcal{L}_{p}}\right) d\left(\widetilde{\tau}, h^{\prime}\right)
\end{aligned}
$$

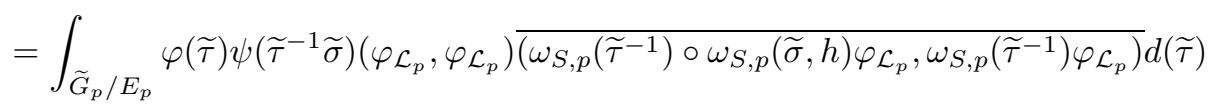

$$
\begin{aligned}
& =(\varphi * \psi)(\widetilde{\sigma}) \overline{\Phi_{S, p}(\widetilde{\sigma}, h)}=(\varphi * \psi)_{J}(\sigma, h) .
\end{aligned}
$$

So $\varphi \mapsto \varphi_{J}$ is a $\mathbb{C}$-algebra homomorphism.

In the next section, we will show that the representation matrix of the inverse mapping of $\varphi \mapsto \varphi_{J}$ with respect to the $\mathbb{C}$-basis $\left\{\varphi_{\alpha, S} \mid \alpha \in \Upsilon\right\}$ of $\mathcal{H}_{J, p}$ and $\mathbb{C}$-basis $\left\{\psi_{\alpha, \nu} \mid \alpha \in \Upsilon\right\}$ of $\mathcal{H}_{p}$ is diagonal. This is the main result of this chapter (see Theorem 2.3.2).

2.2.2. The condition (2) of Appendix B.3 implies that $\Phi_{S, p}$ satisfies an integral equation

$$
\int_{\widetilde{K}_{J, p}} \Phi_{S, p}\left(g k g^{\prime}\right) d k=\Phi_{S, p}(g) \Phi_{S, p}\left(g^{\prime}\right) \quad \text { for all } g, g^{\prime} \in \widetilde{G}_{J, p}
$$

The following proposition is a keystone for the proof of our main result, Theorem 2.3.2.

Proposition. $\Phi_{S, p}(\sigma \widetilde{k} \tau)=\Phi_{S, p}(\sigma) \Phi_{S, p}(\tau)$ for all $\sigma, \tau \in \widetilde{G}_{p} \subset \widetilde{G}_{J, p}$ and $\widetilde{k} \in \widetilde{K}_{J, p}$.

Proof. We can suppose that $\sigma=\widetilde{\mathrm{d}}\left(p^{\alpha}\right)$ and $\tau=\widetilde{\mathrm{d}}\left(p^{\beta}\right)$ for some $\alpha, \beta \in \Upsilon$. Take any element $h^{-1} \widetilde{k}$ of $\widetilde{K}_{J, p}$ where $\widetilde{k}=\mathbf{r}_{\mathcal{L}_{p}}^{\prime}(k)$ with $k \in K_{p}$ and $h=\left(\left(\ell, \ell^{\prime}\right), 0\right) \in H\left[\mathcal{L}_{p}, D\right]$ with $\ell \in \mathcal{L}_{p}, \ell^{\prime} \in \mathcal{L}_{p}^{\prime}$. Then

$$
\begin{aligned}
\Phi_{S, p}\left(\widetilde{\mathrm{d}}\left(p^{\alpha}\right) \cdot h^{-1} \widetilde{k}\right. & \left.\cdot \widetilde{\mathrm{d}}\left(p^{\beta}\right)\right) \\
& =\left(\omega_{S, p}(\widetilde{k}) \circ \omega_{S, p}\left(\widetilde{\mathrm{d}}\left(p^{\beta}\right)\right) \varphi_{\mathcal{L}_{p}}, \Pi_{S, p}(h) \circ \omega_{S, p}\left(\widetilde{\mathrm{d}}\left(p^{-\alpha}\right)\right) \varphi_{\mathcal{L}_{p}}\right) .
\end{aligned}
$$

Put

$$
\begin{aligned}
\varphi(x) & =\left(\Pi_{S, p}(h) \circ \omega_{S, p}\left(\tilde{\mathrm{d}}\left(p^{-\alpha}\right)\right) \varphi_{\mathcal{L}_{p}}\right)(x) \\
& =\mathbf{e}_{p}\left(\left\langle x, \ell^{\prime}\right\rangle_{S}\right) \cdot \eta_{p}^{m|\alpha|} p^{m|\alpha| / 2} \varphi_{\mathcal{L}_{p} p^{\alpha}}(x+\ell) \quad\left(x \in \mathbb{W}_{p}\right)
\end{aligned}
$$

where $\varphi_{\mathcal{L}_{p} p^{\alpha}}$ is the characteristic function of $\mathcal{L}_{p} p^{\alpha}$ in $\mathbb{W}_{p}$. Then for all $(x, y) \in$ $\mathbb{V}_{p}=\mathbb{W}_{p} \oplus \mathbb{W}_{p}^{\prime}$, we have

$$
\begin{aligned}
\theta_{\varphi}(x, y) & =\int_{\mathcal{L}_{p}} \varphi(x+\lambda) \mathbf{e}_{p}\left(\langle\lambda, y\rangle_{S}\right) d_{\mathcal{L}_{p}}(\lambda) \\
& = \begin{cases}\eta_{p}^{m|\alpha|} p^{-m|\alpha| / 2} \mathbf{e}_{p}\left(-\langle x+\ell, y\rangle_{S}\right) & \text { if }(x, y) \in \mathcal{L}_{p} \oplus \mathcal{L}_{p}^{\prime} p^{-\alpha}, \\
0 & \text { otherwise }\end{cases} \\
& =\overline{\Phi_{S, p}\left(\widetilde{\mathrm{d}}\left(p^{\alpha}\right)\right)} \cdot \mathbf{e}_{p}\left(-\langle x+\ell, y\rangle_{S}\right) \sum_{j=1}^{p^{|\alpha|}} \varphi_{\mathcal{L}_{p} \oplus \mathcal{L}_{p}^{\prime}}\left(x, y-\lambda_{j}^{\prime}\right)
\end{aligned}
$$


where $\varphi_{\mathcal{L}_{p} \oplus \mathcal{L}_{p}^{\prime}}$ is the characteristic function of $\mathcal{L}_{p} \oplus \mathcal{L}_{p}^{\prime}$ in $\mathbb{V}_{p}$, and $\left\{\lambda_{j}^{\prime} \mid 1 \leq j \leq p^{|\alpha|}\right\}$ is a complete set of representatives of $\mathcal{L}_{p}^{\prime} p^{-\alpha} / \mathcal{L}_{p}^{\prime}$ such that $\lambda_{1}^{\prime} \in \mathcal{L}_{p}^{\prime}$. Put

$$
\psi(x)=\left(\omega_{S, p}\left(\widetilde{\mathrm{d}}\left(p^{\beta}\right)\right) \varphi_{\mathcal{L}_{p}}\right)(x)=\Phi_{S, p}\left(\widetilde{\mathrm{d}}\left(p^{\beta}\right)\right) \varphi_{\mathcal{L}_{p} p^{-\beta}}(x) \quad\left(x \in \mathbb{W}_{p}\right) .
$$

Then for all $(x, y) \in \mathbb{V}_{p}=\mathbb{W}_{p} \oplus \mathbb{W}^{\prime} p$, we have

$$
\begin{aligned}
\theta_{\psi}(x, y) & =\int_{\mathcal{L}_{p}} \psi(x+\lambda) \mathbf{e}_{p}\left(\langle\lambda, y\rangle_{S}\right) d_{\mathcal{L}_{p}}(\lambda) \\
& = \begin{cases}\Phi_{S, p}\left(\widetilde{\mathrm{d}}\left(p^{\beta}\right)\right) & \text { if }(x, y) \in \mathcal{L}_{p} p^{-\beta} \oplus \mathcal{L}_{p}^{\prime}, \\
0 & \text { otherwise }\end{cases} \\
& =\Phi_{S, p}\left(\widetilde{\mathrm{d}}\left(p^{\beta}\right)\right) \cdot \sum_{i=1}^{p^{|\beta|}} \varphi_{\mathcal{L}_{p} \oplus \mathcal{L}_{p}^{\prime}}\left(x-\lambda_{i}, y\right)
\end{aligned}
$$

where $\left\{\lambda_{i} \mid 1 \leq i \leq p^{|\beta|}\right\}$ is a complete set of representatives of $\mathcal{L}_{p} p^{-\beta} / \mathcal{L}_{p}$ such that $\lambda_{1} \in \mathcal{L}_{p}$. Now we have

$$
\begin{aligned}
& \Phi_{S, p}\left(\widetilde{\mathrm{d}}\left(p^{\alpha}\right) \cdot h^{-1} \widetilde{k} \cdot \widetilde{\mathrm{d}}\left(p^{\beta}\right)\right)=\left(\omega_{S, p}(\widetilde{k}) \theta_{\psi}, \theta_{\varphi}\right) \\
& =\int_{\mathbb{V}_{p}} \theta_{\psi}((x, y) k) \overline{\theta_{\varphi}(x, y)} d_{\mathbb{V}_{p}}(x, y) \\
& =\Phi_{S, p}\left(\widetilde{\mathrm{d}}\left(p^{\alpha}\right)\right) \Phi_{S, p}\left(\widetilde{\mathrm{d}}\left(p^{\beta}\right)\right) \\
& \quad \times \sum_{i=1}^{p^{|\beta|}} \sum_{j=1}^{p^{|\alpha|}} \mathbf{e}_{p}\left(\left\langle\ell, \lambda_{j}^{\prime}\right\rangle_{S}\right) \varphi_{\mathcal{L}_{p} \oplus \mathcal{L}_{p}^{\prime}}\left(\left(0, \lambda_{j}^{\prime}\right) k-\left(\lambda_{i}, 0\right)\right) \cdot \int_{\mathcal{L}_{p} \oplus \mathcal{L}_{p}^{\prime}} \mathbf{e}_{p}\left(\left\langle x, \lambda_{j}^{\prime}\right\rangle_{S}\right) d_{\mathbb{V}_{p}}(x, y) \\
& =\Phi_{S, p}\left(\widetilde{\mathrm{d}}\left(p^{\alpha}\right)\right) \Phi_{S, p}\left(\widetilde{\mathrm{d}}\left(p^{\beta}\right)\right) \cdot \sum_{i=1}^{p^{|\beta|}} \varphi_{\mathcal{L}_{p} \oplus \mathcal{L}_{p}^{\prime}}\left(-\left(\lambda_{i}, 0\right)\right) \\
& =\Phi_{S, p}\left(\widetilde{\mathrm{d}}\left(p^{\alpha}\right)\right) \Phi_{S, p}\left(\widetilde{\mathrm{d}}\left(p^{\beta}\right)\right) . \quad \square
\end{aligned}
$$

\subsection{Isomorphisms of algebras of Hecke operators.}

2.3.1. We will start with investigating a relation between the zonal spherical functions on $G_{J, p}$ and on $\widetilde{G}_{p}$.

Lemma. For any $\omega \in \Omega\left(\widetilde{G}_{p} / / \widetilde{K}_{p}, E_{p} ; \nu_{p}^{m}\right)$, let $\omega_{J}$ be a continuous function on $G_{J, p}$ defined by

$$
\omega_{J}(\sigma, h)=\omega(\widetilde{\sigma}) \Phi_{S, p}(\widetilde{\sigma}, h) \quad \text { for }(\widetilde{\sigma}, h) \in \widetilde{G}_{J, p} \text { such that } \varpi(\widetilde{\sigma})=\sigma .
$$

Then $\omega_{J} \in \Omega\left(G_{J, p} / / K_{J, p}, \mathbb{U}_{p} ; \chi_{S, p}\right)$.

Proof. We shall verify the defining conditions of Appendix B.3. First of all, $\omega_{J}$ is a well-defined and non-zero continuous function on $G_{J, p}$ which satisfies

1) $\omega_{J}\left(k g k^{\prime}\right)=\omega_{J}(g)$ for all $k, k^{\prime} \in K_{J, p}$,

2) $\omega_{J}(g s)=\chi_{S, p}(s) \omega_{J}(g)$ for all $s \in \mathbb{U}_{p}$.

Then the same argument as used in the proof of Proposition 2.1.3 shows that the support of $\omega_{J}$ is contained in $\bigsqcup_{\alpha \in \Upsilon} K_{J, p} \mathrm{~d}\left(p^{\alpha}\right) K_{J, p} \mathbb{U}_{p}$. In particular, $\omega_{J}$ does not 
vanish at

$$
g=(1,(0, y), 0) \cdot \mathrm{d}\left(p^{\alpha}\right) \cdot(1,(x, 0), 0) \in G_{J, p}
$$

only if $(x, y) \in \mathcal{L}_{p} \oplus \mathcal{L}_{p}^{\prime}$. Now we will verify the integral formula

$$
\int_{K_{J, p}} \omega_{J}\left(g k g^{\prime}\right) d k=\omega_{J}(g) \omega_{J}\left(g^{\prime}\right) \quad\left(g, g^{\prime} \in G_{J, p}\right) .
$$

It is enough to show the integral formula for

$$
g=(1,(0, y), 0) \cdot \mathrm{d}\left(p^{\alpha}\right) \cdot(1,(x, 0), 0), \quad g^{\prime}=\left(1,\left(0, y^{\prime}\right), 0\right) \cdot \mathrm{d}\left(p^{\beta}\right) \cdot\left(1,\left(x^{\prime}, 0\right), 0\right)
$$

for some $\alpha, \beta \in \Upsilon$. For any $\ell \in \mathcal{L}_{p}$, we have

$$
\begin{aligned}
\int_{K_{J, p}} \omega_{J}\left(g k g^{\prime}\right) d k & =\int_{K_{J, p}} \omega_{J}\left((1,(\ell, 0), 0) \cdot g k g^{\prime}\right) d k \\
& =\int_{K_{J, p}} \omega_{J}\left(\left(g \cdot\left(1,\left(\ell p^{\alpha}\right), 0\right), D(\ell, y)\right) \cdot k g^{\prime}\right) d k \\
& =\mathbf{e}_{p}\left(\langle\ell, y\rangle_{S}\right) \int_{K_{J, p}} \omega_{J}\left(g k g^{\prime}\right) d k,
\end{aligned}
$$

and for any $\ell^{\prime} \in \mathcal{L}_{p}^{\prime}$, we have

$$
\begin{aligned}
\int_{K_{J, p}} \omega_{J}\left(g k g^{\prime}\right) d k & =\int_{K_{J, p}} \omega_{J}\left(g \cdot\left(1,\left(0, \ell^{\prime}\right), 0\right) \cdot k g^{\prime}\right) d k \\
& =\mathbf{e}_{p}\left(\left\langle x, \ell^{\prime}\right\rangle_{S}\right) \int_{K_{J, p}} \omega_{J}\left(g k g^{\prime}\right) d k .
\end{aligned}
$$

Similarly we have

$$
\begin{aligned}
\int_{K_{J, p}} \omega_{J}\left(g k g^{\prime}\right) d k & =\mathbf{e}_{p}\left(\left\langle\ell, y^{\prime}\right\rangle_{S}\right) \int_{K_{J, p}} \omega_{J}\left(g k g^{\prime}\right) d k \\
& =\mathbf{e}_{p}\left(\left\langle x^{\prime}, \ell^{\prime}\right\rangle_{S}\right) \int_{K_{J, p}} \omega_{J}\left(g k g^{\prime}\right) d k
\end{aligned}
$$

for any $\ell \in \mathcal{L}_{p}$ and $\ell^{\prime} \in \mathcal{L}_{p}^{\prime}$. So if $(x, y) \notin \mathcal{L}_{p} \oplus \mathcal{L}_{p}^{\prime}$ or $\left(x^{\prime}, y^{\prime}\right) \notin \mathcal{L}_{p} \oplus \mathcal{L}_{p}^{\prime}$, then we have

$$
\int_{K J, p} \omega_{J}\left(g k g^{\prime}\right) d k=0=\omega_{J}(g) \omega_{J}\left(g^{\prime}\right) .
$$

Suppose $(x, y),\left(x^{\prime}, y^{\prime}\right) \in \mathcal{L}_{p} \oplus \mathcal{L}_{p}^{\prime}$. Then, by Proposition 2.2.2, we have

$$
\begin{aligned}
\int_{K_{J, p}} \omega_{J}\left(g k g^{\prime}\right) d k & =\int_{K_{J, p}} \omega_{J}\left(\mathrm{~d}\left(p^{\alpha}\right) k \mathrm{~d}\left(p^{\beta}\right)\right) d k \\
& =\int_{\widetilde{K}_{p}} \omega\left(\widetilde{\mathrm{d}}\left(p^{\alpha}\right) k \widetilde{\mathrm{d}}\left(p^{\beta}\right)\right) d k \Phi_{S, p}\left(\widetilde{\mathrm{d}}\left(p^{\alpha}\right)\right) \Phi_{S, p}\left(\widetilde{\mathrm{d}}\left(p^{\beta}\right)\right) \\
& =\omega\left(\widetilde{\mathrm{d}}\left(p^{\alpha}\right)\right) \omega\left(\widetilde{\mathrm{d}}\left(p^{\beta}\right)\right) \Phi_{S, p}\left(\widetilde{\mathrm{d}}\left(p^{\alpha}\right)\right) \Phi_{S, p}\left(\widetilde{\mathrm{d}}\left(p^{\beta}\right)\right) \\
& =\omega_{J}(g) \omega_{J}\left(g^{\prime}\right) .
\end{aligned}
$$

Now the integral formula is proved, and we have $\omega_{J} \in \Omega\left(G_{J, p} / / K_{J, p}, \mathbb{U}_{p} ; \chi_{S, p}\right)$. 
2.3.2. In 2.1.3, we defined $\mathbb{C}$-basis $\left\{\varphi_{\alpha, S} \mid \alpha \in \Upsilon\right\}$ and $\left\{\psi_{\alpha, \nu} \mid \alpha \in \Upsilon\right\}$ of $\mathcal{H}_{J, p}$ and $\mathcal{H}_{p}$, respectively. Define a $\mathbb{C}$-linear isomorphism $T_{p}: \mathcal{H}_{J, p} \stackrel{\sim}{\rightarrow} \mathcal{H}_{p}$ by

$$
T_{p}\left(\varphi_{\alpha, S}\right)=\eta_{p}^{-m|\alpha|} p^{m|\alpha| / 2} \psi_{\alpha, \nu} \quad \text { for all } \alpha \in \Upsilon .
$$

Then our main result in this chapter is

Theorem. 1) $T_{p}$ is a $\mathbb{C}$-algebra isomorphism of $\mathcal{H}_{J, p}$ onto $\mathcal{H}_{p}$ which is the inverse of the isomorphism $\varphi \mapsto \varphi_{J}$ given in Proposition 2.2.1.

2) $\widehat{\omega} \circ T_{p}=\widehat{\omega_{J}}$ for all $\omega \in \Omega\left(\widetilde{G}_{p} / / \widetilde{K}_{p}, E_{p} ; \nu_{p}^{m}\right)$.

Proof. We will prove the second assertion first. For any $\omega \in \Omega\left(\widetilde{G}_{p} / / \widetilde{K}_{p}, E_{p} ; \nu_{p}^{m}\right)$, we have

$$
\begin{aligned}
\widehat{\omega_{J}}\left(\varphi_{\alpha, S}\right) & =\int_{G_{J, p}} \varphi_{\alpha}(g) \omega_{J}(g) d g \\
& =\omega_{J}\left(\mathrm{~d}\left(p^{\alpha}\right)\right) C_{J, \alpha}=\omega\left(\widetilde{\mathrm{d}}\left(p^{\alpha}\right)\right) \Phi_{S, p}\left(\widetilde{\mathrm{d}}\left(p^{\alpha}\right)\right) C_{J, \alpha} \\
\widehat{\omega}\left(\psi_{\alpha, \nu}\right) & =\int_{\widetilde{G}_{p}} \psi_{\alpha}(\sigma) \omega(\sigma) d \sigma \\
& =\omega\left(\widetilde{\mathrm{d}}\left(p^{\alpha}\right)\right) C_{\alpha}
\end{aligned}
$$

where $C_{J, \alpha}$ (resp. $C_{\alpha}$ ) is the volume of $K_{J, p} \mathrm{~d}\left(p^{\alpha}\right) K_{J, p}$ (resp. $\left.\widetilde{K}_{p} \widetilde{\mathrm{d}}\left(p^{\alpha}\right) \widetilde{K}_{p}\right)$ with respect to the Haar measure on $G_{J, p}$ (resp. $\widetilde{G}_{p}$ ). Because of the normalization of the Haar measures, we have

$$
\operatorname{vol}_{\widetilde{G}_{p}}\left(\widetilde{K}_{p} \cap \widetilde{\mathrm{d}}\left(p^{\alpha}\right) \widetilde{K}_{p} \widetilde{\mathrm{d}}\left(p^{\alpha}\right)^{-1}\right)=\operatorname{vol}_{G_{p}}\left(K \cap \mathrm{d}\left(p^{\alpha}\right) K \mathrm{~d}\left(p^{\alpha}\right)^{-1}\right) .
$$

Then we have $C_{\alpha}^{-1} C_{J, \alpha}=p^{m|\alpha|}$. So $\widehat{\omega} \circ T_{p}=\widehat{\omega_{J}}$ for all $\omega \in \Omega\left(\widetilde{G}_{p} / / \widetilde{K}_{p}, E_{p} ; \nu_{p}^{m}\right)$.

Now we will prove the first assertion. For any $\varphi, \psi \in \mathcal{H}_{J, p}$, we have

$$
\widehat{\omega}\left(T_{p}(\varphi * \psi)-T_{p}(\varphi) T_{p}(\psi)\right)=\widehat{\omega_{J}}(\varphi * \psi)-\widehat{\omega_{J}}(\varphi) \widehat{\omega_{J}}(\psi)=0
$$

for all $\omega \in \Omega\left(\widetilde{G}_{p} / / \widetilde{K}_{p}, E_{p} ; \nu_{p}^{m}\right)$. This means that $T_{p}(\varphi * \psi)-T_{p}(\varphi) T_{p}(\psi) \in \bigcap_{\lambda} \operatorname{Ker} \lambda$, where $\lambda$ runs over the surjective $\mathbb{C}$-algebra homomorphism of $\mathcal{H}_{p}$ onto $\mathbb{C}$ (see Appendix B.3). By the isomorphism of Proposition 2.2.1 and by the structure theorem by [Mur], $\mathcal{H}_{p}$ is a domain and is finitely generated as a $\mathbb{C}$-algebra. Then $\bigcap_{\lambda} \operatorname{Ker} \lambda$, which is the intersection of the maximal ideals of $\mathcal{H}_{p}$, is equal to $\{0\}$. So $T_{p}$ is a $\mathbb{C}$-algebra isomorphism. Take a $\varphi \in \mathcal{H}_{p}$. For any $\omega \in \Omega\left(\widetilde{G}_{p} / / \widetilde{K}_{p}, E_{p} ; \nu_{p}^{m}\right)$, we have

$$
\begin{aligned}
& \widehat{\omega} \circ T_{p}\left(\varphi_{J}\right)=\widehat{\omega_{J}}\left(\varphi_{J}\right)=\int_{\widetilde{G}_{J, p} / E_{p} \times \mathbb{U}_{p}} \varphi(\widetilde{\sigma}) \overline{\Phi_{S, p}(\widetilde{\sigma}, h)} \omega(\widetilde{\sigma}) \Phi_{S, p}(\widetilde{\sigma}, h) d(\widetilde{\sigma}, h) \\
& =\int_{\widetilde{G}_{J, p} / E_{p} \times \mathbb{U}_{p}} \varphi(\widetilde{\sigma}) \omega(\widetilde{\sigma})\left(\omega_{S, p}(\widetilde{\sigma}) \circ \Pi_{S, p}(h) \varphi_{\left.\mathcal{L}_{p}, \varphi_{\mathcal{L}_{p}}\right)}\right. \\
& \cdot \overline{\left(\omega_{S, p}(\widetilde{\sigma}) \circ \Pi_{S, p}(h) \varphi_{\mathcal{L}_{p}}, \varphi_{\mathcal{L}_{p}}\right)} d(\widetilde{\sigma}, h) \\
& =\int_{\widetilde{G}_{p} / E_{p}} \varphi(\widetilde{\sigma}) \omega(\widetilde{\sigma})\left(\varphi_{\mathcal{L}_{p}}, \varphi_{\mathcal{L}_{p}}\right) \overline{\left(\omega_{S, p}(\widetilde{\sigma})^{-1} \varphi_{\mathcal{L}_{p}}, \omega_{S, p}(\widetilde{\sigma})^{-1} \varphi_{\mathcal{L}_{p}}\right)} d(\widetilde{\sigma}) \\
& =\widehat{\omega}(\varphi) \text {. }
\end{aligned}
$$

This means that $\lambda \circ T_{p}\left(\varphi_{J}\right)=\lambda(\varphi)$ for all surjective $\mathbb{C}$-algebra homomorphisms $\lambda$ of $\mathcal{H}_{p}$ onto $\mathbb{C}$. Then we have $T_{p}\left(\varphi_{J}\right)=\varphi$, that is, $T_{p}$ is the inverse mapping of $\varphi \mapsto \varphi_{J}$. 
2.3.3. By means of the $\mathbb{C}$-algebra isomorphism $T_{p}$, we can prove the following

Proposition. The correspondence $\omega \mapsto \omega_{J}$ is a bijection of $\Omega\left(\widetilde{G}_{p} / / \widetilde{K}_{p}, E_{p} ; \nu_{p}^{m}\right)$ onto $\Omega\left(G_{J, p} / / K_{J, p}, \mathbb{U}_{p} ; \chi_{S, p}\right)$.

Proof. The mapping $\omega \mapsto \omega_{J}$ is injective, because the support of $\omega$ is contained in that of $\Phi_{S, p}$ (see the proof of Lemma 2.3.1). For any $\omega^{\prime} \in \Omega\left(G_{J, p} / / K_{J, p}, \mathbb{U}_{p} ; \chi_{S, p}\right)$, there exists a surjective $\mathbb{C}$-algebra homomorphism $\lambda$ of $\mathcal{H}_{p}$ onto $\mathbb{C}$ such that $\widehat{\omega^{\prime}}=$ $\lambda \circ T_{p}$. Then there exists an $\omega \in \Omega\left(\widetilde{G}_{p} / / \widetilde{K} p, E_{p} ; \nu_{p}^{m}\right)$ such that $\widehat{\omega}=\lambda$. So we have $\widehat{\omega_{J}}=\widehat{\omega^{\prime}}$ and finally $\omega_{J}=\omega^{\prime}$.

\subsection{Unitary class-one representations.}

2.4.1. When composed with the canonical projection $\widetilde{G}_{J, p} \rightarrow G_{J, p}$, a unitary representation of $G_{J, p}$ is identified with a unitary representation of $\widetilde{G}_{J, p}$. Under this identification, the correspondence $\pi \mapsto \pi_{J} \otimes \omega_{S, p}$ of Theorem 1.3.3 gives a bijection of $\mathcal{R}\left(\widetilde{G}_{p}, E ; \nu_{p}^{m}\right)$ onto $\mathcal{R}\left(G_{J, p}, \mathbb{U}_{p} ; \chi_{S, p}\right)$. On the other hand, since $\mathcal{H}_{J, p}$ and $\mathcal{H}_{p}$ are commutative, the general theory of zonal spherical function (see Appendix B.4) gives a bijection of $\mathcal{R}\left(G_{J, p} / / K_{J, p}, \mathbb{U}_{p} ; \chi_{S, p}\right)$ onto $\Omega^{+}\left(G_{J, p} / / K_{J, p}, \mathbb{U}_{p} ; \chi_{S, p}\right)$ and of $\mathcal{R}\left(\widetilde{G}_{p} / / \widetilde{K}_{p}, E_{p} ; \nu_{p}^{m}\right)$ onto $\Omega^{+}\left(\widetilde{G}_{p} / / \widetilde{K} p, E_{p} ; \nu_{p}^{m}\right)$, respectively. We have the following proposition.

Proposition. A bijection of $\mathcal{R}\left(\widetilde{G}_{p} / / \widetilde{K}_{p}, E_{p} ; \nu_{p}^{m}\right)$ onto $\mathcal{R}\left(G_{J, p} / / K_{J, p}, \mathbb{U}_{p} ; \chi_{S, p}\right)$ is given by correspondence $\tau \mapsto \pi=\tau_{J} \otimes \omega_{S, p}$. We have $\omega_{\pi}=\left(\omega_{\tau}\right)_{J}$.

Proof. Take any $\pi \in \mathcal{R}\left(G_{J, p} / / K_{J, p}, \mathbb{U}_{p} ; \chi_{S, p}\right)$ with the representation space $H_{\pi}$. Let $F$ be the complex vector space consisting of the continuous $\mathbb{C}$-linear mapping $T$ of $L^{2}\left(\mathbb{W}_{p}\right)$ to $H_{\pi}$ such that $T \circ \Pi_{S, p}(h)=\pi(h) \circ T$ for all $h \in H\left[\mathbb{V}_{p}, D\right]$. Then $F$ is a complex Hilbert space with respect to the norm $|T|=\sup _{\varphi \in L^{2}\left(\mathbb{W}_{p}\right)}|T \varphi| /|\varphi|$. Define a unitary representation $\tau$ of $\widetilde{G}_{p}$ on $F$ by $\tau(\widetilde{\sigma}) T=\pi(\sigma) \circ T \circ \omega_{S, p}(\widetilde{\sigma})^{-1}$ for all $\widetilde{\sigma} \in \widetilde{G}_{p}$ such that $\varpi(\widetilde{\sigma})=\sigma$. Then $\tau \in \mathcal{R}\left(\widetilde{G}_{p} / / \widetilde{K}_{p}, E_{p} ; \nu_{p}^{m}\right)$ and $\tau_{J} \otimes \omega_{S, p}=\pi$ by the unitary isomorphism $F \widehat{\otimes} L^{2}\left(\mathbb{W}_{p}\right) \stackrel{\sim}{\rightarrow} H_{\pi}$ defined by $T \otimes \varphi \mapsto T \varphi$. Take a $K_{J, p}$-invariant vector $u_{0} \in H_{\pi}$ such that $\left|u_{0}\right|=1$. Now $T \mapsto T \varphi_{\mathcal{L}_{p}}$ gives a unitary isomorphism of $F$ onto the subspace of $H_{\pi}$ consisting of the $H\left[\mathcal{L}_{p}, D\right]$ invariant vectors. Then there exists uniquely a $T_{0} \in F$ such that $T_{0} \varphi_{\mathcal{L}_{p}}=u_{0}$. Since $\left(\tau(k) T_{0}\right) \varphi_{\mathcal{L}_{p}}=u_{0}$ for all $k \in \widetilde{K}_{p}, T_{0} \in F$ is a $\widetilde{K}_{p}$-invariant vector. So $\tau \in \mathcal{R}\left(\widetilde{G}_{p} / / \widetilde{K}_{p}, E_{p} ; \nu_{p}^{m}\right)$. The zonal spherical function $\omega_{\pi}$ of $\pi$ is

$$
\begin{aligned}
\left(\pi(g) u_{0}, u_{0}\right) & =\left(\tau(\widetilde{\sigma}) T_{0}, T_{0}\right)\left(\omega_{S, p}(\widetilde{\sigma}, h) \varphi_{\mathcal{L}_{p}}, \varphi_{\mathcal{L}_{p}}\right) \\
& =\omega_{\tau}(\widetilde{\sigma}) \Phi_{S, p}(\widetilde{\sigma}, h) \\
& =\left(\omega_{\tau}\right)_{J}(g)
\end{aligned}
$$

for all $g=(\sigma, h) \in G_{J, p}$ with $\widetilde{\sigma} \in \widetilde{G}_{p}$ such that $\varpi(\widetilde{\sigma})=\sigma$.

2.4.2. Proposition 2.4.1 says that the correspondence $\omega \mapsto \omega_{J}$ of Proposition 2.3.3 gives a bijection of $\Omega^{+}\left(\widetilde{G}_{p} / / \widetilde{K}_{p}, E_{p} ; \nu_{p}^{m}\right)$ onto $\Omega^{+}\left(G_{J, p} / / K_{J, p}, \mathbb{U}_{p} ; \chi_{S, p}\right)$. Then consider the bounded zonal spherical functions. The correspondence $\omega \mapsto \omega_{J}$ gives an injection of $\Omega^{b}\left(\widetilde{G}_{p} / / \widetilde{K}_{p}, E_{p} ; \nu_{p}^{m}\right)$ into $\Omega^{b}\left(G_{J, p} / / K_{J, p}, \mathbb{U}_{p} ; \chi_{S, p}\right)$. Is it surjective? This question is related with the growth rate problem stated in [Sat3, Remark 3 to Theorem 3]. 


\section{Chapter 3. Global Theory}

We will suppose the conditions (A),(B),(C) of 1.1.2 and the condition (D) $M^{*} S \subset M$ where $M^{*}=\{u \in U \mid\langle u, M\rangle \subset \mathbb{Z}\}$ is the dual lattice of $M$. Under the condition $(\mathrm{D})$, we have $\rho\left(S p\left(L_{p}\right)\right) \subset S p\left(\mathcal{L}_{p}\right)$ for all $p \leq \infty$ (see Proposition 1.4.11). We will fix a finite set $\Sigma$ of finite places of $\mathbb{Q}$ such that $2 \in \Sigma$ and

1) $\left\{u_{1}, \cdots, u_{m}\right\}$ is a $\mathbb{Z}_{p}$-basis of $M_{p}$,

2) $M_{p}^{*}=M_{p}$

for all finite places $p$ of $\mathbb{Q}$ outside $\Sigma$.

\subsection{Global correspondence of automorphic forms.}

3.1.1. For any finite place $p$ of $\mathbb{Q}$, let $K_{p}$ be a open subgroup of $S p\left(L_{p}\right)$ such that $K_{p}=S p\left(L_{p}\right)$ for all $p \notin \Sigma$. Let $K_{\infty}$ be a maximal compact subgroup of $G_{\infty}=S p\left(V_{\infty}\right)$ which is the isotropy subgroup of $z_{0} \in \mathfrak{H}_{W}$. Put $K=\prod_{p \leq \infty} K_{p}$ which is a compact subgroup of $G_{A}$. The Haar measure $d_{G_{p}}(\sigma)$ of $G_{p}$ for $p<\infty$ is normalized so that $\operatorname{vol}\left(K_{p}\right)=1$.

Let

$$
H\left[\mathbb{V}_{A}, D\right]=\mathbb{V}_{A} \times \mathbb{U}_{A}=\prod_{p \leq \infty}^{\prime} H\left[\mathbb{V}_{p}, D\right]
$$

be the restricted direct product of $\left\{H\left[\mathbb{V}_{p}, D\right]\right\}_{p \leq \infty}$ with respect to $\left\{H\left[\mathcal{L}_{p}, D\right]\right\}_{p<\infty}$. Here $H\left[\mathcal{L}_{p}, D\right]=\left(\mathcal{L}_{p} \oplus \mathcal{L}_{p}^{\prime}\right) \times \frac{1}{4} \mathcal{M}_{p}$ is an open compact subgroup of $H\left[\mathbb{V}_{p}, D\right]$.

$G_{A}$ acts on $H\left[\mathbb{V}_{A}, D\right]$, and the semi-direct product $G_{J, A}=G_{A} \ltimes H\left[\mathbb{V}_{A}, D\right]$ is the restricted direct product of $\left\{G_{J, p}\right\}_{p \leq \infty}$ with respect to $K_{J, p}=K_{p} \ltimes H\left[\mathcal{L}_{p}, D\right]$ $(p<\infty)$. Then $K_{J}=K_{\infty} \times \prod_{p<\infty} K_{J, p}$ is a compact subgroup of $G_{J, A}$.

Put $\widetilde{K}_{p}=\mathbf{r}_{L_{p}}^{\prime}\left(K_{p}\right)$ for $2<p<\infty$, and $\widetilde{K}_{p}=\varpi_{p}^{-1}\left(K_{p}\right)$ for $p=2, \infty$. Then $\widetilde{K}=\prod_{p \leq \infty} \widetilde{K}_{p}$ is a compact subgroup of $\widetilde{G}_{A}$. The Haar measure $d_{\widetilde{G}_{p}}(\widetilde{\sigma})$ of $\widetilde{G}_{p}$ for $p<\infty$ is normalized so that $\operatorname{vol}\left(\widetilde{K}_{p}\right)=1$ for $p \neq 2$, and $\operatorname{vol}\left(\widetilde{K}_{2}\right)=2$.

$\widetilde{G}_{A}$ acts on $H\left[\mathbb{V}_{A}, D\right]$ via

$$
\varpi=\prod_{p \leq \infty} \varpi_{p}: \widetilde{G}_{A} \rightarrow G_{A} .
$$

Then the semi-direct product $\widetilde{G}_{J, A}=\widetilde{G}_{A} \ltimes H\left[\mathbb{V}_{A}, D\right]$ is the restricted direct product of $\left\{\widetilde{G}_{J, p}\right\}_{p \leq \infty}$ with respect to $\widetilde{K}_{J, p}=\widetilde{K}_{p} \ltimes H\left[\mathcal{L}_{p}, D\right](p<\infty)$.

3.1.2. $H\left[\mathbb{V}_{A}, D\right]$ has a unique irreducible unitary representation $\Pi_{S}$ such that $\Pi_{S}(0, t)=\mathbf{e}(\operatorname{tr}(S \cdot t))$ for all $t \in \mathbb{U}_{A}$. It is realized on $L^{2}\left(\mathbb{W}_{A}\right)$ by

$$
\left(\Pi_{S}(h) \varphi\right)(w)=\mathbf{e}\left(\operatorname{tr}(S \cdot t)+\frac{1}{2}\langle x, y\rangle_{S}+\langle w, y\rangle_{S}\right) \varphi(w+x) \quad\left(\varphi \in L^{2}\left(\mathbb{W}_{A}\right)\right)
$$

for $h=((x, y), t) \in H\left[\mathbb{V}_{A}, D\right]\left(x \in \mathbb{W}_{A}, y \in \mathbb{W}_{A}^{\prime}\right)$. Then $\Pi_{S}$ is unitarily equivalent to the restricted tensor product of $\left\{\Pi_{S, p}\right\}_{p \leq \infty}$ with respect to $\left\{\varphi_{\mathcal{L}_{p}}\right\}_{p<\infty}$ where $\varphi_{\mathcal{L}_{p}} \in L^{2}\left(\mathbb{W}_{p}\right)$ is the characteristic function of $\mathcal{L}_{p}$ in $\mathbb{W}_{p}$ (see Appendix A.5 for the definition of the restricted tensor product of representations). $\Pi_{S}$ is unitarily isomorphic to the induced representation $\operatorname{Ind}\left(H\left[\mathbb{V}_{A}, D\right], \Lambda ; \xi_{S}\right)$ where $\Lambda=\mathbb{V} \times \mathbb{U}_{A}$ 
and $\xi_{S}(\lambda)=\mathbf{e}(\operatorname{tr}(S \cdot t))(\lambda=(x, t) \in \Lambda)$ is a character of $\Lambda$. The unitary intertwining mapping $\varphi \mapsto \Theta_{\varphi}$ is given by

$$
\Theta_{\varphi}(h)=\sum_{\ell \in \mathbb{W}} \varphi(x+\ell) \mathbf{e}\left(\operatorname{tr}(S \cdot t)+\frac{1}{2}\langle x, y\rangle_{S}+\langle\ell, y\rangle_{S}\right)
$$

for any Schwartz-Bruhat function $\varphi \in \mathcal{S}\left(\mathbb{W}_{A}\right)$ and $h=((x, y), t) \in H\left[\mathbb{V}_{A}, D\right]$.

3.1.3. The Weil representation $\omega_{p}$ is realized on $L^{2}\left(\mathbb{W}_{p}\right)$ for all $p \leq \infty$. Let $\omega_{A}$ be the restricted tensor product of $\left\{\omega_{p}\right\}_{p \leq \infty}$ with respect to $\left\{\varphi_{\mathcal{L}_{p}}\right\}_{p<\infty}$. Then $\omega_{A}$ is realized on $L^{2}\left(\mathbb{W}_{A}\right)$. It is also realized on $\operatorname{Ind}\left(H\left[\mathbb{V}_{A}, D\right], \Lambda ; \xi_{S}\right)$. The intertwining mapping is given by (3.1.2.1). $\omega_{A}$ is a unitary representation of $\prod_{p \leq \infty}^{\prime} \widetilde{S p}\left(\mathbb{V}_{p}\right)$, the restricted direct product of $\left\{\widetilde{S p}\left(\mathbb{V}_{p}\right)\right\}_{p \leq \infty}$ with respect to $\left\{\widetilde{S p}\left(\mathcal{L}_{p}\right)\right\}_{p<\infty}$.

The irreducible unitary representation $\omega_{S, p}$ of $\widetilde{G}_{J, p}$ is realized on $L^{2}\left(\mathbb{W}_{p}\right)$ for all $p \leq \infty$. Let $\omega_{S, A}$ be the restricted tensor product of $\left\{\omega_{S, p}\right\}_{p \leq \infty}$ with respect to $\left\{\varphi_{\mathcal{L}_{p}}\right\}_{p<\infty}$. Then $\omega_{S, A}$ is an irreducible unitary representation of $\widetilde{G}_{J, A}$, and

$$
\omega_{S, A}(\widetilde{\sigma}, h)=\omega_{A}(\widetilde{\rho}(\widetilde{\sigma})) \circ \Pi_{S}(h) \text { for all }(\widetilde{\sigma}, h) \in \widetilde{G}_{J, A}=\widetilde{G}_{A} \ltimes H\left[\mathbb{V}_{A}, D\right],
$$

where

$$
\widetilde{\rho}: \widetilde{G}_{A} \rightarrow \prod_{p \leq \infty}^{\prime} \widetilde{S p}\left(\mathbb{V}_{p}\right) \quad\left(\text { defined by }\left(\sigma_{p}\right)_{p \leq \infty} \mapsto\left(\widetilde{\rho}_{p}\left(\sigma_{p}\right)\right)_{p \leq \infty}\right)
$$

is a continuous group homomorphism which is well-defined by Proposition 2.1.1.

3.1.4. Let $G_{A}^{\Sigma}$ (resp. $\widetilde{G}_{A}^{\Sigma}$ ) be the subgroup of $G_{A}$ (resp. $\widetilde{G}_{A}$ ) consisting of the $\left(\sigma_{p}\right)_{p \leq \infty}$ such that $\sigma_{p} \in K_{p}$ (resp. $\left.\sigma_{p} \in \widetilde{K}_{p}\right)$ for all $p \in \Sigma$. Put $G_{J, A}^{\Sigma}=G_{A}^{\Sigma} \ltimes$ $H\left[\mathbb{V}_{A}, D\right]$ and $\widetilde{G}_{J, A}^{\Sigma}=\widetilde{G}_{A}^{\Sigma} \ltimes H\left[\mathbb{V}_{A}, D\right]$.

Put $G_{J, p}^{\Sigma}=G_{J, p}$ if $p \notin \Sigma$, and $G_{J, p}^{\Sigma}=K_{p} \ltimes H\left[\mathbb{V}_{p}, D\right]$ if $p \in \Sigma$. Then $G_{J, A}^{\Sigma}$ is the restricted direct product of $\left\{G_{J, p}^{\Sigma}\right\}_{p \leq \infty}$ with respect to $\left\{K_{J, p}\right\}_{p<\infty}$. Similarly put $\widetilde{G}_{J, p}^{\Sigma}=\widetilde{G}_{J, p}$ if $p \notin \Sigma$, and $\widetilde{G}_{J, p}^{\Sigma}=\widetilde{K}_{p} \ltimes H\left[\mathbb{V}_{p}, D\right]$ if $p \in \Sigma$. Then $\widetilde{G}_{J, A}^{\Sigma}$ is the restricted direct product of $\left\{\widetilde{G}_{J, p}^{\Sigma}\right\}_{p \leq \infty}$ with respect to $\left\{\widetilde{K}_{J, p}\right\}_{p<\infty}$.

Put $\omega_{S, A}^{\Sigma}=\left.\omega_{S, A}\right|_{\widetilde{G}_{J, A}^{\Sigma}}$ which is an irreducible unitary representation of $\widetilde{G}_{J, A}^{\Sigma}$.

For any unitary representation $\pi$ of $\widetilde{G}_{A}^{\Sigma}$, let us denote by $\pi_{J}$ the composition of $\pi$ with the canonical projection $\widetilde{G}_{J, A}^{\Sigma}$ onto $\widetilde{G}_{A}^{\Sigma}$. Then, similar to Theorem 1.3.3, we have

Theorem. $\pi \mapsto \pi_{J} \otimes \omega_{S, A}^{\Sigma}$ gives a bijection between the set of the unitary equivalence classes of the unitary representations of $\widetilde{G}_{A}^{\Sigma}$ and the set of the unitary equivalence classes of the unitary representations $\tau$ of $\widetilde{G}_{J, A}^{\Sigma}$ such that $\tau(t)=\mathbf{e}(\operatorname{tr}(S \cdot t))$ for all $t \in Z\left(\widetilde{G}_{J, A}^{\Sigma}\right)=\mathbb{U}_{A} . \pi_{J} \otimes \omega_{S, A}^{\Sigma}$ is irreducible if and only if $\pi$ is. $\pi_{J} \otimes \omega_{S, A}^{\Sigma}$ is square-integrable modulo the center if and only if $\pi$ is square-integrable.

3.1.5. Put $G_{\mathbb{Q}}^{\Sigma}=S p(V) \cap G_{A}^{\Sigma}$ (resp. $\widetilde{G}_{\mathbb{Q}}^{\Sigma}=\widetilde{S p}(V) \cap \widetilde{G}_{A}^{\Sigma}$ ) which is a discrete subgroup of $G_{A}^{\Sigma}$ (resp. $\left.\widetilde{G}_{A}^{\Sigma}\right)$. Put $G_{J, \mathbb{Q}}^{\Sigma}=G_{\mathbb{Q}}^{\Sigma} \ltimes H[\mathbb{V}, D]$ which is a discrete subgroup of $G_{J, A}^{\Sigma}$. Then $G_{J, \mathbb{Q}}^{\Sigma} \cdot \mathbb{U}_{A}=G_{\mathbb{Q}}^{\Sigma} \ltimes \Lambda$ is a closed unimodular subgroup of $G_{J, A}^{\Sigma}$. Define a unitary character $\mathbf{1} \otimes \xi_{S}$ of $G_{J, \mathbb{Q}}^{\Sigma} \cdot \mathbb{U}_{A}=G_{\mathbb{Q}}^{\Sigma} \ltimes \Lambda$ by

$$
\left(\mathbf{1} \otimes \xi_{S}\right)(\gamma, \lambda)=\xi_{S}(\lambda) \quad \text { for } \gamma \in G_{\mathbb{Q}}^{\Sigma}, \lambda \in \Lambda .
$$


Consider the induced representation $\pi=\operatorname{Ind}\left(G_{J, A}^{\Sigma}, G_{J, \mathbb{Q}}^{\Sigma} \cdot \mathbb{U}_{A} ; \mathbf{1} \otimes \xi_{S}\right)$. Let $\widetilde{\pi}$ be the composition of $\pi$ with the canonical projection $\widetilde{G}_{J, A}^{\Sigma} \rightarrow G_{J, A}^{\Sigma}$. Then we have

$$
\widetilde{\pi}=\operatorname{Ind}\left(\widetilde{G}_{J, A}^{\Sigma}, \widetilde{G}_{J, \mathbb{Q}}^{\Sigma} \cdot \mathbb{U}_{A} \cdot E^{\Sigma} ; \mathbf{1} \otimes \xi_{S}\right) .
$$

Here we put $E^{\Sigma}=\left(\prod_{p \leq \infty} E_{p}\right) \cap \widetilde{G}_{A}^{\Sigma}=\operatorname{Ker}\left(\varpi^{\Sigma}\right)$ with $\varpi^{\Sigma}=\left.\varpi\right|_{\widetilde{G}_{A}^{\Sigma}}$ and $\mathbf{1} \otimes \xi_{S}$ is regarded as a character of $\widetilde{G}_{J, \mathbb{Q}}^{\Sigma} \cdot \mathbb{U}_{A} \cdot E^{\Sigma}$ via the canonical projection $\widetilde{G}_{J, A}^{\Sigma} \rightarrow G_{J, A}^{\Sigma}$.

We have $\widetilde{G}_{\mathbb{Q}}^{\Sigma} \cdot E^{\Sigma}=\varpi^{\Sigma-1}\left(G_{\mathbb{Q}}^{\Sigma}\right)$. Define a character $\varepsilon_{\Sigma}$ of $\widetilde{G}_{\mathbb{Q}}^{\Sigma} \cdot E^{\Sigma}$ by

$$
J(\widetilde{\gamma})=\left(1, \varepsilon_{\Sigma}(\widetilde{\gamma})\right) \cdot \mathbf{r}_{\mathbb{Q}}(\gamma) \quad\left(\gamma=\varpi(\widetilde{\gamma}) \in G_{\mathbb{Q}}^{\Sigma}\right) .
$$

More explicitly

$$
\varepsilon_{\Sigma}(\widetilde{\gamma})=\prod_{p \leq \infty} \lambda_{p} \text { for } \widetilde{\gamma}=\gamma_{0} \cdot\left(1, \lambda_{p}\right)_{p \leq \infty} \text { with } \gamma_{0} \in \widetilde{G}_{\mathbb{Q}}^{\Sigma},\left(1, \lambda_{p}\right)_{p \leq \infty} \in E^{\Sigma} .
$$

We have $\left.\omega_{S, A}^{\Sigma}\right|_{E^{\Sigma} \times \mathbb{U}_{A}}=\varepsilon_{\Sigma}^{m} \otimes \xi_{S}$. Put $\tau=\operatorname{Ind}\left(\widetilde{G}_{A}^{\Sigma}, \widetilde{G}_{\mathbb{Q}}^{\Sigma} \cdot E^{\Sigma} ; \varepsilon_{\Sigma}^{m}\right)$. Then we have

Theorem. $\tau \otimes \omega_{S, A}^{\Sigma}$ is unitarily equivalent to $\widetilde{\pi}$ by the unitary mapping $\varphi \otimes \psi \mapsto$ $\varphi \otimes \psi$ defined by

$$
(\varphi \otimes \psi)(\sigma, h)=(\tau(\sigma) \varphi)(1) \cdot\left(\omega_{S, A}^{\Sigma}(\sigma, h) \psi\right)(1) .
$$

Here $\omega_{S, A}^{\Sigma}$ is realized on $\operatorname{Ind}\left(H\left[\mathbb{V}_{A}, D\right], \Lambda ; \xi_{S}\right)$.

This theorem is proved by using an argument similar to the proof of Theorem 1.4.1.

Remark. The unitary representations of $G_{J, A}^{\Sigma}$ are regarded as unitary representations of $\widetilde{G}_{J, A}^{\Sigma}$ via the canonical projection $\widetilde{G}_{J, A}^{\Sigma} \rightarrow G_{J, A}^{\Sigma}$. Then the correspondence $\pi \mapsto \pi_{J} \otimes \omega_{S, A}^{\Sigma}$ of Theorem 3.1.4 gives a bijection between the set of the unitary equivalence classes of the unitary representations $\pi$ of $\widetilde{G}_{A}^{\Sigma}$ such that $\left.\pi\right|_{E^{\Sigma}}=\varepsilon_{\Sigma}^{m}$ and the set of the unitary equivalence classes of the unitary representations $\tau$ of $G_{J, A}^{\Sigma}$ such that $\tau(t)=\mathbf{e}(\operatorname{tr}(S \cdot t))$ for all $t \in Z\left(G_{J, A}^{\Sigma}\right)=\mathbb{U}_{A}$.

3.1.6. Let $\delta=\bigotimes_{p \leq \infty} \delta_{p}$ be an irreducible unitary representation of $K$ such that

1) $\delta_{p}=\mathbf{1}_{K_{p}}$ is the trivial representation of $K_{p}$ for all finite $p \notin \Sigma$,

2) $\delta_{p}$ is a finite order character of $K_{p}$ for all $p \in \Sigma$,

\begin{tabular}{|c|c|c|c|c|c|c|c|}
\hline 1 & 2 & $\cdots$ & & $\ldots$ & & $\cdots$ & $\ell_{1}$ \\
\hline 1 & 2 & $\cdots$ & & $\ldots$ & $\ell_{2}$ & & \\
\hline & & $\cdots$ & & $\cdots$ & & & \\
\hline \begin{tabular}{|l|l|} 
\\
\end{tabular} & 2 & $\cdots$ & $\ell_{n}$ & & & & \\
\hline
\end{tabular}

3) $\delta_{\infty}$ corresponds to the Young diagram (given below) with $\ell_{1} \geq \ell_{2} \geq \cdots \geq$ $\ell_{n}>n+m / 2$.

The representation space of $\delta$ is denoted by $V_{\delta}=V_{\delta_{\infty}}$.

As remarked at the beginning of this chapter, the condition (D) implies $\rho\left(S p\left(L_{2}\right)\right) \subset$ $S p\left(\mathcal{L}_{2}\right)$. Then we have $\widetilde{\rho}_{2}\left(\widetilde{S p}\left(L_{2}\right)\right) \subset \widetilde{S p}\left(\mathcal{L}_{2}\right)$ by the definition of $\widetilde{S p}\left(L_{2}\right)$. Define an irreducible unitary representation $\widetilde{\delta}=\bigotimes_{p \leq \infty} \widetilde{\delta}_{p}$ of $\widetilde{K}$ by

1) $\widetilde{\delta}_{p}=\left(\varepsilon_{\mathcal{L}, p} \circ \widetilde{\rho}_{p}\right) \otimes\left(\delta_{p} \circ \varpi_{p}\right)$ for all $p<\infty$,

2) $\widetilde{\delta}_{\infty}=\operatorname{det}^{-m / 2} \otimes\left(\delta_{\infty} \circ \varpi_{\infty}\right)$. 
Note that $\widetilde{\delta}_{p}=\delta_{p} \circ \varpi_{p}$ for all $2<p<\infty$. Then $\widetilde{\delta}_{p}$ is the trivial representation for all finite $p \notin \Sigma$.

For all finite $p$, define a unitary character $\delta_{J, p}$ of $K_{J, p}$ by

$$
\delta_{J, p}(k, h)=\delta_{p}(k) \cdot \xi_{S, p}(h)^{-1} \quad \text { for } k \in K_{p}, h \in H\left[\mathcal{L}_{p}, D\right] .
$$

Note that $\delta_{J, p}$ is the trivial character for all finite $p \notin \Sigma$. Let $\delta_{J}=\delta_{\infty} \otimes \bigotimes_{p<\infty} \delta_{J, p}$ be an irreducible unitary representation of $K_{J}$ on the representation space $V_{\delta_{\infty}}$.

3.1.7. Let $\tau_{p}$ be an irreducible unitary representation of $\widetilde{G}_{p}$ or $\widetilde{K}_{p}(p \leq \infty)$ such that

1) $\tau_{p} \in \mathcal{R}\left(\widetilde{G}_{p} / / \widetilde{K}_{p}, E_{p} ; \nu_{p}^{m}\right)$ for all finite $p \notin \Sigma$,

2) $\tau_{\infty}=\pi_{\delta_{\infty} \otimes \delta_{0}^{-m}}$ is the holomorphic discrete series of $\widetilde{G}_{\infty}$ with minimal $\widetilde{K}_{\infty^{-}}$ type $\delta_{\infty} \otimes \delta_{0}^{-m}$ constructed in $\mathbf{1 . 4 . 5}$

3) $\tau_{p}=\widetilde{\delta}_{p}$ for all $p \in \Sigma$.

For all finite $p \notin \Sigma$, choose a $\widetilde{K}_{p}$-invariant vector $u_{p}$ of unit length in the representation space of $\tau_{p}$. Recall that the space of the $\widetilde{K}_{P}$-invariant vectors in $\tau_{p}$ is one-dimensional for all finite $p \notin \Sigma$. Let $\tau=\bigotimes_{p \leq \infty}^{\prime} \tau_{p}$ be the restricted tensor product of $\left\{\tau_{p}\right\}_{p \leq \infty}$ with respect to $\left\{u_{p}\right\}_{p \notin \Sigma \cup\{\infty\}}$. Then $\tau$ is an irreducible unitary representation of $\widetilde{G}_{A}^{\Sigma}$ such that the multiplicity of $\widetilde{\delta}$ in $\tau_{\widetilde{K}}$ is equal to one. We have $\left.\tau\right|_{E^{\Sigma}}=\left.\varepsilon_{\Sigma}^{m}\right|_{E^{\Sigma}}$.

3.1.8. Define an irreducible unitary representation $\pi_{p}$ of $G_{J, p}^{\Sigma}(p \leq \infty)$ by

1) $\pi_{p}=\tau_{p} \otimes \check{\omega}_{S, p}$ for all $p \notin \Sigma$, where $\check{\omega}_{S, p}$ is the contragredient representation of $\omega_{S, p}$

2) $\pi_{p}=\widetilde{\delta}_{p} \otimes \check{\omega}_{S, p}$ for all $p \in \Sigma$.

Here if $\left.\widetilde{\delta}_{2}\right|_{E_{2}}=\nu_{2}^{m}$ so $\widetilde{\delta}_{2} \otimes \check{\omega}_{S, 2}$ is trivial on $E_{2}$, then $\pi_{2}=\widetilde{\delta}_{2} \otimes \check{\omega}_{S, 2}$ is regarded as a representation of $G_{J, 2}^{\Sigma}=K_{2} \ltimes H\left[\mathbb{V}_{2}, D\right]$. On the other hand, for all odd $p \in \Sigma$, if the covering mapping $\varpi_{p}$ gives an isomorphism $\widetilde{K}_{p} \sim K_{p}$, then $\pi_{p}=\widetilde{\delta}_{p} \otimes \check{\omega}_{S, p}$ is regarded as a representation of $G_{J, p}^{\Sigma}=K_{p} \ltimes H\left[\mathbb{V}_{p}, D\right]$. $\pi_{\infty}$ is the holomorphic discrete series $\pi_{S, \infty}$ of $G_{J, \infty}$ constructed in 1.4.6.

For all finite $p \notin \Sigma$, the space of the $K_{J, p}$-invariant vectors in $\pi_{p}$ is onedimensional. Choose a $K_{J, p}$-invariant vector $v_{p}$ of $\pi_{p}$ of unit length. Let $\pi=$ $\bigotimes_{p \leq \infty}^{\prime} \pi_{p}$ be the restricted tensor product of $\left\{\pi_{p}\right\}_{p \leq \infty}$ with respect to $\left\{v_{p}\right\}_{p \notin \Sigma \cup\{\infty\}}$. Then $\pi$ is an irreducible unitary representation of $G_{J, A}$ such that the multiplicity of $\delta_{J}$ in $\left.\pi\right|_{K_{J}}$ is equal to one. We have $\left.\pi\right|_{\mathbb{U}_{A}}=\left.\xi_{A}^{-1}\right|_{\mathbb{U}_{A}}$.

3.1.9. For any finite place $p$ of $\mathbb{Q}$, let $\kappa_{p}$ be a character of $\widetilde{K}_{J, p}=\widetilde{K}_{p} \ltimes H\left[\mathcal{L}_{p}, D\right]$ such that

1) $\kappa_{2}=\left(\varepsilon_{\mathcal{L}, 2} \circ \widetilde{\rho}_{2}\right) \otimes \xi_{S, 2}$,

2) $\kappa_{p}=\mathbf{1}_{\widetilde{K}_{p}} \otimes \xi_{S, p}$, for all odd $p \in \Sigma$,

3) $\kappa_{p}=\mathbf{1}_{\widetilde{K}_{J, p}}$ for all finite $p \notin \Sigma$.

Recall that $\varphi_{\mathcal{L}_{p}} \in L^{2}\left(\mathbb{W}_{p}\right)$ is a $\mathbb{C}$-basis of the $\kappa_{p}$-isotypic component of $\omega_{S, p}$. Put $\kappa_{\infty}=\operatorname{det}^{-m / 2}$. Then $\kappa=\bigotimes_{p \leq \infty} \kappa_{p}$ is a character of $\widetilde{K}_{J}$. Let $\varphi=\prod_{p \leq \infty} \varphi_{p} \in$ $\mathcal{S}\left(\mathbb{W}_{A}\right)$ be a Schwartz-Bruhat function such that $\varphi_{p}=\varphi_{\mathcal{L}_{p}}$ for all $p<\infty$ :

$$
\varphi_{\infty}(x)=\operatorname{det}\left(2 \operatorname{Im} z_{0}\right)^{m / 4} \mathbf{e}_{\infty}\left(\frac{1}{2}\left\langle x, x z_{0}\right\rangle_{S}\right) \quad\left(x \in \mathbb{W}_{\infty}\right) .
$$


Then, by the intertwining mapping (3.1.2.1), $\Theta_{\varphi} \in \operatorname{Ind}\left(H\left[\mathbb{V}_{A}, D\right], \Lambda ; \xi_{A}\right)$ is a unitary $\mathbb{C}$-basis of the $\kappa$-isotypic component of $\omega_{S, A}$. Put $\Theta_{S}(g)=\left(\omega_{S, A}(g) \Theta_{\varphi}\right)(1)$ $\left(g \in G_{J, A}\right)$.

3.1.10. Let us denote by $\mathcal{A}\left(K_{J} ; \pi, \delta_{J}, S\right)$ the space of the automorphic forms on $G_{J, A}^{\Sigma}$ associated with the data

$$
(G, K, \Gamma, E ; \xi, \pi, \delta)=\left(G_{J, A}^{\Sigma}, K_{J}, G_{J, \mathbb{Q}}^{\Sigma} \cdot \mathbb{U}_{A}, \mathbb{U}_{A} ; \mathbf{1} \otimes \xi_{S}^{-1}, \pi, \delta_{J}\right)
$$

in the sense of Appendix A.2. Let us also denote by $\mathcal{A}(\widetilde{K} ; \tau, \widetilde{\delta})$ the space of the automorphic forms on $\widetilde{G}_{A}^{\Sigma}$ associated with the data

$$
(G, K, \Gamma, E ; \xi, \pi, \delta)=\left(\widetilde{G}_{A}^{\Sigma}, \widetilde{K}, \widetilde{G}_{\mathbb{Q}}^{\Sigma} \cdot E^{\Sigma}, E^{\Sigma} ; \varepsilon_{\Sigma}^{m}, \tau, \widetilde{\delta}\right) .
$$

Let us denote by $\widetilde{\pi}$ (resp. $\widetilde{\delta}_{J}$ ) the composition of $\pi$ (resp. $\delta_{J}$ ) with the canonical projection $\widetilde{G}_{J, A} \rightarrow G_{J, A}$ (resp. $\widetilde{K}_{J} \rightarrow K_{J}$ ). Then, by the definitions of $\tau$ and $\pi$, we have $\widetilde{\pi}=\tau_{J} \otimes \check{\omega}_{S, A}$. The $\widetilde{\delta}_{J}$-isotypic component in $\widetilde{\pi}$ is the tensor product of the $\widetilde{\delta}$-isotypic component in $\tau$ and the $\check{\kappa}$-isotypic component in $\check{\omega}_{S, A}$. Then, by Theorem 3.1.5 and arguments similar to those in the proof of Theorem 1.4.8, we have the following theorem.

Theorem. $A \mathbb{C}$-linear isometry $F \mapsto f_{F}$ from $\mathcal{A}\left(K_{J} ; \pi, \delta_{J}, S\right)$ onto $\mathcal{A}(\widetilde{K} ; \tau, \widetilde{\delta})$ is defined by

$$
f_{F}(\widetilde{\sigma})=\int_{\Lambda \backslash H\left[\mathbb{V}_{A}, D\right]} F(\sigma, h) \overline{\Theta_{S}(\widetilde{\sigma}, h)} d \dot{h} .
$$

Here $\widetilde{\sigma} \in \widetilde{G}_{A}^{\Sigma}$ is an element such that $\varpi(\widetilde{\sigma})=\sigma \in G_{A}^{\Sigma}$.

3.2. The action of Hecke operators on the infinite part. Throughout this section, we will fix the compact groups and their representations defined in 3.1.1 and 3.1.6, respectively.

3.2.1. Put $\Gamma=S p(V) \cap\left(G_{\infty} \times \prod_{p<\infty} K_{p}\right)$ which is identified with a discrete subgroup of $G_{\infty}$ via the projection to $G_{\infty}$. Put $\widetilde{\Gamma}=\varpi_{\infty}^{-1}(\Gamma)$ which is a discrete subgroup of $\widetilde{G}_{\infty}$.

Proposition. 1) $\widetilde{G}_{A}^{\Sigma}=\widetilde{G}_{\mathbb{Q}}^{\Sigma} \cdot\left(\widetilde{G}_{\infty} \times \prod_{p<\infty} \widetilde{K}_{p}\right)$,

2) $\left(\gamma, T_{p}\right)_{p \leq \infty} \mapsto\left(\gamma, T_{\infty}\right)$ gives an isomorphism

$$
\widetilde{G}_{\mathbb{Q}}^{\Sigma} \cap\left(\widetilde{G}_{\infty} \times \prod_{p<\infty} \widetilde{K}_{p}\right) \stackrel{\sim}{\rightarrow} \widetilde{\Gamma} .
$$

Proof. 1) It is enough to show

$$
\widetilde{G}_{A}=\widetilde{S p}(V) \cdot\left(\widetilde{G}_{\infty} \times \prod_{p<\infty} \widetilde{K}_{p}\right) .
$$

Take any $\widetilde{\sigma}=\left(\sigma_{p}, T_{p}\right)_{p \leq \infty} \in \widetilde{G}_{A}$. By the strong approximation theorem, we have

$$
G_{A}=S p(V) \cdot\left(G_{\infty} \times \prod_{p<\infty} K_{p}\right) .
$$


So $G_{A} \ni\left(\sigma_{p}\right)_{p<\infty}=\gamma \cdot\left(\tau_{\infty},\left(k_{p}\right)_{P<\infty}\right)$ with some $\gamma \in S p(V), \tau_{\infty} \in G_{\infty}$ and $k_{p} \in$ $K_{p}$. Put $\widetilde{k}_{p}=\left(k_{p}, T_{p}^{\prime}\right)=\mathbf{r}_{L_{p}}^{\prime}\left(k_{p}\right) \in \widetilde{K}_{p}$ for $2<p<\infty, \widetilde{k}_{2}=\left(k_{2}, T_{2}^{\prime}\right) \in \varpi_{2}^{-1}\left(k_{2}\right) \subset$ $\widetilde{K}_{2}$ and $\widetilde{\tau}_{\infty}=\left(\tau_{\infty}, T_{\infty}^{\prime}\right) \in \varpi_{\infty}^{-1}\left(\tau_{\infty}\right) \subset \widetilde{G}_{\infty}$. Then there exists an $\varepsilon \in \mathbb{C}^{1}$ such that $\bigotimes_{p \leq \infty}\left(T_{p} T_{p}^{\prime-1}\right)=\varepsilon \cdot \mathbf{r}_{W}(\gamma)$ because $J\left(\widetilde{\sigma} \cdot\left(\widetilde{\tau}_{\infty},\left(\widetilde{k}_{p}\right)_{p<\infty}\right)^{-1}\right) \in \widetilde{S p}\left(V_{A}\right)$ is projected onto $\gamma \in S p(V)$. Replacing $T_{2}^{\prime}$ with $\varepsilon \cdot T_{2}^{\prime}$, we have $\widetilde{\sigma} \in \widetilde{S p}(V) \cdot\left(\widetilde{\tau}_{\infty},\left(\widetilde{k}_{p}\right)_{p<\infty}\right)$.

2) Let $\left(\sigma, T_{p}\right)_{p \leq \infty} \in \widetilde{S p}(V) \cap\left(\widetilde{G}_{\infty} \times \prod_{p<\infty} \widetilde{K}_{p}\right)$ be such that $\left(\sigma, T_{\infty}\right)=1$. Then $\sigma=1$ and $T_{p}=\mathbf{r}_{L_{p}}(\sigma)=1$ for all $2<p<\infty$. Finally we have $\bigotimes_{p \leq \infty} T_{p}=$ $\mathbf{r}_{W}(\sigma)=1$, so $T_{2}=1$. Hence $\left(\sigma, T_{p}\right)_{p \leq \infty} \mapsto\left(\sigma, T_{\infty}\right)$ is an injective group homomorphism into $\widetilde{\Gamma}$. On the other hand, take any $\left(\gamma, T_{\infty}\right) \in \widetilde{\Gamma}$. Then $\gamma \in K_{p}$ for all $p<\infty$, so put $\left(\gamma, T_{p}\right)=\mathbf{r}_{L_{p}}^{\prime}(\gamma) \in \widetilde{K}_{p}$ for all $2<p<\infty$, and choose any $\left(\gamma, T_{2}\right) \in \varpi_{2}^{-1}(\gamma) \subset \widetilde{K}_{2}$. There exists an $\varepsilon \in \mathbb{C}^{1}$ such that $\mathbf{r}_{W}(\gamma)=\varepsilon \cdot \bigotimes_{p \leq \infty} T_{p}$. Replacing $T_{2}$ with $\varepsilon \cdot T_{2}$, we have $\left(\gamma, T_{p}\right)_{p \leq \infty} \in \widetilde{S p}(V) \cap\left(\widetilde{G}_{\infty} \times \prod_{p<\infty} \widetilde{K}_{p}\right)$.

Define a finite order character $\alpha$ of $\Gamma$ by $\alpha(\gamma)=\prod_{p<\infty} \delta_{p}(\gamma)$.

3.2.2. Let $\pi_{\infty}=\pi_{\delta_{\infty}, S}$ be the holomorphic discrete series of $G_{J, \infty}$ constructed in 1.4.6 (see also 3.1.8). Let us denote by $\mathcal{M}^{\Sigma}\left(K_{J} ; \delta_{J}, S\right)$ the complex vector space of the continuous $V_{\delta}$-valued functions $F$ on $G_{J, A}^{\Sigma}$ satisfying the conditions

1) $F(\gamma g)=F(g)$ for all $\gamma \in G_{J, \mathbb{Q}}^{\Sigma}$,

2) $F(\lambda g)=\xi_{S}(\lambda) F(g)$ for all $\lambda \in \Lambda$,

3) $\int_{G_{J, \mathbb{Q}}^{\Sigma} \mathbb{U}_{A} \backslash G_{J, A}^{\Sigma}}|F(g)|^{2} d \dot{g}<\infty$,

4) $F(g k)=\delta_{J}\left(k^{-1}\right) F(g)$ for all $k \in K_{J}$,

5) $F$ satisfies the integral equation

$$
\int_{G_{J, \infty} / \mathbb{U}_{\infty}} F\left(g h^{-1}\right) \varphi(h) d \dot{h}=\widehat{\psi}_{\pi_{\infty}, \delta_{\infty}}(\varphi) F(g)
$$

for all $\varphi \in C_{c}\left(G_{J, \infty} / \mathbb{U}_{\infty}, \chi_{S, \infty}^{-1}, \delta_{J, \infty}\right)^{0}$.

We have

$$
G_{J, A}^{\Sigma}=G_{J, \mathbb{Q}}^{\Sigma} \cdot\left(G_{J, \infty} \times \prod_{p<\infty} K_{J, p}\right) .
$$

So, for any $F \in \mathcal{A}\left(\Gamma_{J}, \alpha ; \delta_{\infty}, S\right)$, define a $V_{\delta}$-valued function $F_{A}$ on $G_{J, A}^{\Sigma}$ by

$$
F_{A}(g)=\prod_{p<\infty} \delta_{J, p}\left(k_{p}^{-1}\right) \cdot F\left(g_{\infty}\right) \quad \begin{gathered}
\text { for } \\
\text { with }
\end{gathered} \quad \begin{gathered}
g=\gamma \cdot\left(g_{\infty},\left(k_{p}\right)_{p<\infty}\right) \in G_{J, \mathbb{Q}}^{\Sigma}, g_{\infty}^{\Sigma} \in G_{J, \infty}, k_{p} \in K_{J, p} .
\end{gathered}
$$

Then $F_{A}$ is well-defined, and $F \mapsto F_{A}$ gives a $\mathbb{C}$-linear isometry from $\mathcal{A}\left(\Gamma_{J}, \alpha ; \delta_{\infty}, S\right)$ onto $\mathcal{M}^{\Sigma}\left(K_{J} ; \delta_{J}, S\right)$. In particular, $\mathcal{M}^{\Sigma}\left(K_{J} ; \delta_{J}, S\right)$ is a finite dimensional complex Hilbert space with the inner product

$$
\left(F, F^{\prime}\right)=\int_{G_{J, A}^{\Sigma} / \mathbb{U}_{A}}\left(F(g), F^{\prime}(g)\right)_{\delta} d \dot{g}
$$

For any finite $p \notin \Sigma$, the algebra of the local Hecke operators

$$
\check{\mathcal{H}}_{J, p}=C_{c}\left(G_{J, p} / / K_{J, p}, \mathbb{U}_{p} ; \chi_{S, p}^{-1}\right)
$$


acts on $\mathcal{M}^{\Sigma}\left(K_{J} ; \delta_{J}, S\right)$ by convolution

$$
(F * \varphi)(g)=\int_{G_{J, p} / \mathbb{U}_{p}} F\left(g x^{-1}\right) \varphi(x) d \dot{x} \quad \text { for all } \varphi \in \check{\mathcal{H}}_{J, p}
$$

3.2.3. If we take the local representations $\pi_{p}$ and the global representation $\pi$ defined in 3.1.8, we have the following proposition.

Proposition. $\mathcal{A}\left(K_{J} ; \pi, \delta_{J}, S\right)$ is the subspace of $F \in \mathcal{M}^{\Sigma}\left(K_{J} ; \delta, S\right)$ such that

$$
F * \varphi=\widehat{\omega}_{\pi_{p}}(\varphi) F \quad \text { for all } \varphi \in \check{\mathcal{H}}_{J, p}
$$

with all finite $p \notin \Sigma$. Here $\omega_{\pi_{p}} \in \Omega^{+}\left(G_{J, p} / / K_{J, p}, \mathbb{U}_{p} ; \chi_{S, p}^{-1}\right)$ is the zonal spherical function associated with $\pi_{p} \in \mathcal{R}\left(G_{J, p} / / K_{J, p}, \mathbb{U}_{p} ; \chi_{S, p}^{-1}\right)$ (see Appendix B.4), and

$$
\widehat{\omega}_{\pi_{p}}(\varphi)=\int_{G_{J, p} / \mathbb{U}_{p}} \omega_{\pi_{p}}(g) \varphi(g) d \dot{g}
$$

(see Appendix B.3).

Proof. Proposition A.5 in Appendix A implies that $\mathcal{A}\left(K_{J} ; \pi, \delta_{J}, S\right)$ is the subspace of $F \in \mathcal{M}^{\Sigma}\left(K_{J} ; \delta_{J}, S\right)$ such that

$$
\int_{G_{J, p}^{\Sigma} / \mathbb{U}_{p}} F\left(g h^{-1}\right) \varphi(h) d \dot{h}=\widehat{\psi}_{\pi_{p}, \delta_{p}}(\varphi) F(g)
$$

for all $\varphi \in C_{c}\left(G_{J, p}^{\Sigma} / \mathbb{U}_{p}, \chi_{S, p}^{-1}, \delta_{J, p}\right)^{0}$ with all finite $p$. For all finite $p \notin \Sigma$,

$$
C_{c}\left(G_{J, p}^{\Sigma} / \mathbb{U}_{p}, \chi_{S, p}^{-1}, \delta_{J, p}\right)^{0}=C_{c}\left(G_{J, p} / / K_{J, p}, \mathbb{U}_{p} ; \chi_{S, p}^{-1}\right), \quad \text { and } \quad \psi_{\pi_{p}, \delta_{J, p}}=\omega_{\pi_{p}} .
$$

Take any $p \in \Sigma$. For any $\varphi \in C_{c}\left(G_{J, p}^{\Sigma} / \mathbb{U}_{p}, \chi_{S, p}^{-1}, \delta_{J, p}\right)^{0}$, we have

$$
\operatorname{supp}(\varphi) \subset K_{J, p} \cdot \mathbb{U}_{p}
$$

by the same argument as that used in the proof of Proposition 2.1.3. Then we have

$$
\int_{G_{J, p}^{\Sigma} / \mathbb{U}_{p}} F\left(g h^{-1}\right) \varphi(h) d \dot{h}=\varphi(1) F(g) .
$$

On the other hand,

$$
\psi_{\pi_{p}, \delta_{J, p}}(g)=\delta_{p}(k) \xi_{S, p}^{-1}(h) \times \begin{cases}1 & \text { if }(x, y) \in \mathcal{L}_{p} \oplus \mathcal{L}_{p}^{\prime}, \\ 0 & \text { otherwise }\end{cases}
$$

for all $g=(k, h) \in G_{J, p}^{\Sigma}=K_{p} \ltimes H\left[\mathbb{V}_{p}, D\right]$ with $h=((x, y), t) \in H\left[\mathbb{V}_{p}, D\right]$. Then we have

$$
\widehat{\psi}_{\pi_{p}, \delta_{J, p}}(\varphi)=\varphi(1) \quad \text { for all } \varphi \in C_{c}\left(G_{J, p}^{\Sigma} / \mathbb{U}_{p}, \chi_{S, p}^{-1}, \delta_{J, p}\right)^{0} .
$$

Then we have the required conditions.

3.2.4. Let $\tau_{\infty}$ be the holomorphic discrete series of $\widetilde{G}_{\infty}$ defined in 3.1.7. Let us denote by $\mathcal{M}^{\Sigma}(\widetilde{K} ; \widetilde{\delta})$ the complex vector space of the continuous $V_{\delta}$-valued functions $f$ on $\widetilde{G}_{A}^{\Sigma}$ satisfying the conditions

1) $f(\gamma \sigma)=\varepsilon_{\Sigma}^{m}(\gamma) f(\sigma)$ for all $\gamma \in \widetilde{G}_{\mathbb{Q}}^{\Sigma} \cdot E^{\Sigma}$,

2) $\int_{\widetilde{G}_{\mathbb{Q}}^{\Sigma} E^{\Sigma} \backslash \widetilde{G}_{A}^{\Sigma}}|f(\sigma)|^{2} d \dot{\sigma}<\infty$,

3) $f(\sigma k)=\widetilde{\delta}\left(k^{-1}\right) f(\sigma)$ for all $k \in \widetilde{K}$, 
4) $f$ satisfies the integral equations

$$
\int_{\widetilde{G}_{\infty} / E_{\infty}} f\left(\sigma x^{-1}\right) \varphi(x) d \dot{x}=\widehat{\psi}_{\tau_{\infty}, \widetilde{\delta}_{\infty}}(\varphi) f(\sigma)
$$

for all $\varphi \in C_{c}\left(\widetilde{G}_{\infty} / E_{\infty}, \nu_{\infty}, \widetilde{\delta}_{\infty}\right)^{0}$.

By Proposition 3.2.1, we have

$$
\widetilde{G}_{A}^{\Sigma}=\widetilde{G}_{\mathbb{Q}}^{\Sigma} \cdot\left(\widetilde{G}_{\infty} \times \prod_{p<\infty} \widetilde{K}_{p}\right)
$$

So, for any $f \in \mathcal{A}\left(\widetilde{\Gamma}, \widetilde{\alpha} ; \widetilde{\delta}_{\infty}\right)$, define a $V_{\delta}$-valued function $f_{A}$ on $\widetilde{G}_{A}^{\Sigma}$ by

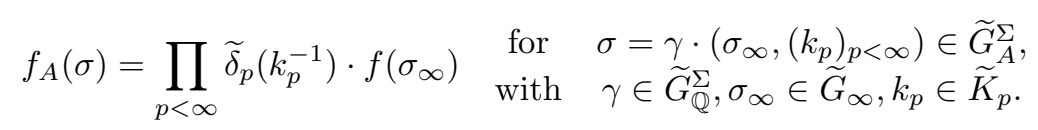

Then $f_{A}$ is well-defined, and $f \mapsto f_{A}$ gives a $\mathbb{C}$-linear isomorphism from $\mathcal{A}\left(\widetilde{\Gamma}, \widetilde{\alpha} ; \widetilde{\delta}_{\infty}\right)$ onto $\mathcal{M}^{\Sigma}(\widetilde{K} ; \widetilde{\delta})$. Here we use Proposition 1.2.6 and the statement 2) of Proposition 3.2.1. Because of the special normalization of the Haar measure of $\widetilde{G}_{2}$, the mapping $f \mapsto f_{A}$ is isometric. In particular, $\mathcal{M}^{\Sigma}(\widetilde{K} ; \widetilde{\delta})$ is a finite dimensional complex Hilbert space with respect to the inner product

$$
\left(f, f^{\prime}\right)=\int_{\widetilde{G}_{A}^{\Sigma} / E^{\Sigma}}\left(f(\sigma), f^{\prime}(\sigma)\right)_{\delta} d \dot{\sigma} .
$$

For any finite $p \notin \Sigma$, the algebra of the local Hecke operators

$$
\mathcal{H}_{p}=C_{c}\left(\widetilde{G}_{p} / / \widetilde{K}_{p}, E_{p} ; \nu_{p}^{m}\right)
$$

acts on $\mathcal{M}^{\Sigma}(\widetilde{K} ; \widetilde{\delta})$ by convolution

$$
(f * \varphi)(\sigma)=\int_{\widetilde{G}_{p} / E_{p}} f\left(\sigma x^{-1}\right) \varphi(x) d \dot{x} \quad \text { for all } \varphi \in \mathcal{H}_{p} .
$$

3.2.5. If we take the local representations $\tau_{p}$ and the global representation $\tau$ defined in 3.1.7, we have the following proposition which is proved by the same argument as that used for Proposition 3.2.3.

Proposition. $\mathcal{A}(\widetilde{K} ; \tau, \widetilde{\delta})$ is the subspace of $f \in \mathcal{M}^{\Sigma}(\widetilde{K} ; \widetilde{\delta})$ such that

$$
f * \varphi=\widehat{\omega}_{\tau_{p}}(\varphi) f \quad \text { for all } \varphi \in \mathcal{H}_{p}
$$

with all finite $p \notin \Sigma$. Here $\omega_{\tau_{p}} \in \Omega^{+}\left(\widetilde{G}_{p} / / \widetilde{K}_{p}, E_{p} ; \nu_{p}^{m}\right)$ is the zonal spherical function associated with $\tau_{p} \in \mathcal{R}\left(\widetilde{G}_{p} / / \widetilde{K}_{p}, E_{p} ; \nu_{p}^{m}\right)$ (see Appendix B.4), and

$$
\widehat{\omega}_{\tau_{p}}(\varphi)=\int_{\widetilde{G}_{p} / E_{p}} \omega_{\tau_{p}}(\sigma) \varphi(\sigma) d \dot{\sigma}
$$

(see Appendix B.3). 
3.2.6. Now we will show the main theorem in this section.

Theorem. For any $F \in \mathcal{M}^{\Sigma}\left(K_{J} ; \delta_{J}, S\right)$, put

$$
f_{F}(\widetilde{\sigma})=\int_{\Lambda \backslash H\left[\mathbb{V}_{A}, D\right]} F(\sigma, h) \overline{\Theta_{S}(\widetilde{\sigma}, h)} d \dot{h}
$$

where $\widetilde{\sigma} \in \widetilde{G}_{A}^{\Sigma}$ is an element such that $\varpi(\widetilde{\sigma})=\sigma \in G_{A}^{\Sigma}$. Then $F \mapsto f_{F}$ is a $\mathbb{C}$-linear isometry from $\mathcal{M}^{\Sigma}\left(K_{J} ; \delta_{J}, S\right)$ onto $\mathcal{M}^{\Sigma}(\widetilde{K} ; \widetilde{\delta})$ which is equivariant with respect to the actions of Hecke operators:

$$
f_{F * \varphi}=f_{F} * T_{p}(\varphi) \quad \text { for all } \varphi \in \check{\mathcal{H}}_{J, p}
$$

with all finite $p \notin \Sigma$.

Proof. We will embed $\widetilde{G}_{\infty}$ in $\widetilde{G}_{A}^{\Sigma}$ by $\sigma_{\infty}=\left(\sigma_{\infty}, 1,1, \cdots\right)$. For any $f \in \mathcal{A}\left(\widetilde{\Gamma}, \widetilde{\alpha} ; \widetilde{\delta}_{\infty}\right)$, we have $f_{A}\left(\sigma_{\infty}\right)=f\left(\sigma_{\infty}\right)$ for all $\sigma_{\infty} \in \widetilde{G}_{\infty}$. We have also

$$
\Theta_{S}\left(\sigma_{\infty}, h\right)=\Theta_{S, \infty}\left(\sigma_{\infty}, h_{\infty}\right) \quad \text { for all } h \in \mathbb{V}_{\infty} \times \prod_{p<\infty}\left(\mathcal{L}_{p} \oplus \mathcal{L}_{p}^{\prime}\right) \subset H\left[\mathbb{V}_{A}, D\right]
$$

Then, for any $F \in \mathcal{M}^{\Sigma}\left(K_{J} ; \delta_{J}, S\right)$, we have

$$
\begin{aligned}
f_{F_{A}}\left(\widetilde{\sigma}_{\infty}\right) & =\int_{\mathbb{V} \cap\left(\mathbb{V}_{\infty} \times \prod_{p<\infty} \mathcal{L}_{p} \oplus \mathcal{L}_{p}^{\prime}\right) \backslash \mathbb{V}_{\infty} \times \prod_{p<\infty} \mathcal{L}_{p} \oplus \mathcal{L}_{p}^{\prime}} F_{A}\left(\sigma_{\infty}, h^{\sigma_{\infty}}\right) \overline{\Theta_{S}\left(\widetilde{\sigma}_{\infty}, h^{\sigma_{\infty}}\right)} d \dot{h} \\
& =\int_{\Lambda \backslash H\left[\mathbb{V}_{\infty}, D\right]} F\left(\sigma_{\infty}, h^{\left.\sigma_{\infty}\right)} \overline{\Theta_{S, \infty}\left(\widetilde{\sigma}_{\infty}, h^{\sigma_{\infty}}\right)} d \dot{h}\right. \\
& =f_{F}\left(\widetilde{\sigma}_{\infty}\right) .
\end{aligned}
$$

Hence $F \mapsto f_{F}$ is a $\mathbb{C}$-linear isometry from $\mathcal{M}^{\Sigma}\left(K_{J} ; \delta_{J}, S\right)$ onto $\mathcal{M}^{\Sigma}(\widetilde{K} ; \widetilde{\delta})$ by Theorem 1.4.8.

Fix a finite $p \notin \Sigma$. For any $\varphi \in \check{\mathcal{H}}_{J, p}$, we have

$$
\left(F * \varphi, F^{\prime}\right)=\left(F, F^{\prime} * \varphi^{*}\right) \text { for all } F, F^{\prime} \in \mathcal{M}^{\Sigma}\left(K_{J} ; \delta_{J}, S\right) .
$$

Here we put $\varphi^{*}(g)=\overline{\varphi\left(g^{-1}\right)}$. On the other hand we have $\varphi_{\alpha, S}^{*}=\varphi_{\alpha, S}$ for all $\alpha \in \Upsilon$ (see 2.1.4 for the notation). Because $\check{\mathcal{H}}_{J, p}$ is commutative, $\bigsqcup_{p \notin \Sigma \cup\{\infty\}} \check{\mathcal{H}}_{J, p}$ acts on $\mathcal{M}^{\Sigma}\left(K_{J} ; \delta_{J}, S\right)$ as normal linear operators which are commutative with each other. Then there exists an orthonormal $\mathbb{C}$-basis $\left\{F_{1}, \cdots, F_{n}\right\}$ of $\mathcal{M}^{\Sigma}\left(K_{J} ; \delta_{J}, S\right)$ such that each $F_{j}$ is a common eigenfunction of the linear operators $\bigsqcup_{p \notin \Sigma \cup\{\infty\}} \check{\mathcal{H}}_{J, p}$; that is, there exists a surjective $\mathbb{C}$-algebra homomorphism

$$
\lambda_{J, p}^{(j)}: \check{\mathcal{H}}_{J, p} \rightarrow \mathbb{C} \quad \text { such that } F_{j} * \varphi=\lambda_{J, p}^{(j)}(\varphi) F_{j} \text { for all } \varphi \in \check{\mathcal{H}}_{J, p} .
$$

Fix a non-zero $\alpha \in V_{\delta}^{*}$. Then $F_{j, \alpha}(g)=\left\langle F_{j}(g), \alpha\right\rangle$ is a non-zero element of

$$
\operatorname{Ind}\left(G_{J, A}^{\Sigma}, G_{J, \mathbb{Q}}^{\Sigma} \cdot \mathbb{U}_{A} ; \mathbf{1} \otimes \xi_{S}\right),
$$

and $\omega_{J, p}^{(j)}(g)=\left(g \cdot F_{j, \alpha}, F_{j, \alpha}\right)\left|F_{j, \alpha}\right|^{-2}$ is an element of $\Omega^{+}\left(G_{J, p} / / K_{J, p}, \mathbb{U}_{p} ; \chi_{S, p}\right)$ such that $\widehat{\omega}_{J, p}^{(j)}=\lambda_{J, p}^{(j)}$. Let $\pi_{p}^{(j)} \in \mathcal{R}\left(G_{J, p} / / K_{J, p}, \mathbb{U}_{p} ; \chi_{S, p}^{-1}\right)$ be the class-one representation of $G_{J, p}$ whose contragredient representation corresponds to $\omega_{J, p}^{(j)}$. By Proposition 2.4.1, we have $\pi_{J, p}^{(j)}=\left(\tau_{p}^{(j)}\right)_{J} \otimes \check{\omega}_{S, p}$ with a class-one representation 
$\tau_{p}^{(j)} \in \mathcal{R}\left(\widetilde{G}_{p} / / \widetilde{K}_{p}, E_{p} ; \nu_{p}^{m}\right)$ of $\widetilde{G}_{p}$. Let $\omega_{p}^{(j)} \in \Omega^{+}\left(\widetilde{G}_{p} / / \widetilde{K}_{p}, E_{p} ; \nu_{p}^{m}\right)$ be the zonal spherical function associated with $\tau_{p}^{(j)}$.

Let $\tau^{(j)}$ (resp. $\pi^{(j)}$ ) be the global representation defined by the local representations $\left\{\tau_{p}^{(j)}\right\}_{p \notin \Sigma \cup\{\infty\}}$ (resp. $\left\{\pi_{p}^{(j)}\right\}_{p \notin \Sigma \cup\{\infty\}}$ ) as in 3.1.7 (resp. 3.1.8). Then $F_{j}$ is an element of $\mathcal{A}\left(K_{J} ; \pi^{(j)}, \delta_{J}, S\right)$. By Theorem 3.1.10,

$$
f_{j}(\widetilde{\sigma})=\int_{\Lambda \backslash H\left[\mathbb{V}_{A}, D\right]} F_{j}(\sigma, h) \overline{\Theta_{S}(\widetilde{\sigma}, h)} d \dot{h}
$$

is an element of $\mathcal{A}(\widetilde{K} ; \tau, \widetilde{\delta})$. In particular, $\left\{f_{1}, \cdots, f_{n}\right\}$ is an orthonormal $\mathbb{C}$-basis of $\mathcal{M}^{\Sigma}(\widetilde{K} ; \widetilde{\delta})$ such that

$$
f_{j} * \varphi=\widehat{\omega}_{p}^{(j)}(\varphi) f_{j} \quad \text { for all } \varphi \in \mathcal{H}_{p} \text { with all finite } p \notin \Sigma .
$$

Then we have

$$
f_{j} * T_{p}(\varphi)=f_{F_{j} * \varphi} \quad \text { for all } \varphi \in \check{\mathcal{H}}_{J, p} \text { with all finite } p \notin \Sigma,
$$

because we have $\widehat{\omega}_{p}^{(j)} \circ T_{p}=\widehat{\omega}_{J, p}^{(j)}$ by Theorem 2.3.2. The proof is completed.

3.3. Classical theory revisited. In this section, we will reconsider some results of $[\mathrm{E}-\mathrm{Z}]$ and $[\mathrm{Ibu}]$ from our point of view.

3.3.1. Put $V=\mathbb{Q}^{2 m}$ (row vectors) and $[x, y]=x \cdot J_{n} \cdot{ }^{t} y$ with $J_{n}=\left[\begin{array}{cc}0 & 1_{n} \\ -1_{n} & 0\end{array}\right]$. Define a polarization $V=W \oplus W^{\prime}$ by

$$
W=\left\{(x, 0) \in V \mid x \in \mathbb{Q}^{n}\right\}, \quad W^{\prime}=\left\{(0, y) \in V \mid y \in \mathbb{Q}^{n}\right\} .
$$

Take $\mathbb{Z}$-lattices

$$
L=\left\{(x, 0) \in V \mid x \in \mathbb{Z}^{n}\right\} \subset W, \quad L^{\prime}=\left\{(0, y) \in V \mid y \in \mathbb{Z}^{n}\right\} \subset W^{\prime} .
$$

Put $U=\mathbb{Q}$ (one-dimensional $\mathbb{Q}$-vector space) and $\langle u, v\rangle=u \cdot v$ for $u, v \in U$. Take a $\mathbb{Z}$-lattice $M=\mathbb{Z} \subset U$.

$\mathbb{V}=\operatorname{Hom}_{\mathbb{Q}}(U, V)\left(\right.$ resp. $\left.\mathbb{U}=\operatorname{Sym}_{\mathbb{Q}}(U)\right)$ is canonically identified with $V$ (resp. $U)$, and $D(x, y)=[x, y]$ in the notation of 1.1.1. Take $S=1 \in \mathbb{U}=U$. Then $D_{S}(x, y)=[x, y]$, and $\mathbb{W}=\operatorname{Hom}_{\mathbb{Q}}(U, W)\left(\operatorname{resp} . \mathbb{W}^{\prime}=\operatorname{Hom}_{\mathbb{Q}}\left(U, W^{\prime}\right)\right)$ is canonically identified with $W\left(\operatorname{resp} . W^{\prime}\right)$. Also $\mathcal{L}=\operatorname{Hom}_{\mathbb{Z}}(M, L)\left(\operatorname{resp} . \mathcal{L}^{\prime}=\operatorname{Hom}_{\mathbb{Q}}\left(M, L^{\prime}\right)\right)$ is canonically identified with $L$ (resp. $\left.L^{\prime}\right)$. Then the conditions (A), (B), (C) and (D) are fulfilled, and $\Sigma=\{2\}$ for a $\mathbb{Z}$-basis $\left\{u_{1}=1\right\}$ of $M$.

The group $\Gamma_{0}=S p(L)$ consists of the $\left[\begin{array}{ll}a & b \\ c & d\end{array}\right] \in S p(n, \mathbb{Z})$ such that the diagonal elements of $a \cdot{ }^{t} b$ and $c \cdot{ }^{t} d$ are all even.

3.3.2. Put

$$
\theta_{r}(z, w)=\sum_{\ell \in \mathbb{Z}^{n}} \mathbf{e}_{\infty}\left\{\left(\ell+\frac{r}{2}\right) z^{t}\left(\ell+\frac{r}{2}\right)+2\left(\ell+\frac{r}{2}\right)^{t} w\right\} \quad \text { for all } r \in \mathbb{Z}^{n} .
$$

Then we have

$$
\vartheta_{S}(z, w)=\sum_{r \in \mathbb{Z}^{n} / 2 \mathbb{Z}^{n}} \theta_{r}(2 z, w)
$$


and the orthogonality relations

$$
\int_{\mathbb{C}^{n} / L_{z}^{\prime}} \theta_{r}(2 z, w) \overline{\theta_{s}(2 z, w)} \kappa_{S}(z, w) d_{z}(w)= \begin{cases}\operatorname{det}(2 \cdot \operatorname{Im} z)^{-1 / 2}, & \text { if } r=s \\ 0, & \text { if } r \neq s\end{cases}
$$

where $L_{z}^{\prime}=\left\{x z+y \mid x \in 2 \mathbb{Z}^{n}, y \in \mathbb{Z}^{n}\right\}$ is a $\mathbb{Z}$-lattice in $\mathbb{C}^{n}$.

3.3.3. Let $S_{\ell-1 / 2}\left(\Gamma_{0}(4)\right)$ be the space of Siegel cusp forms of weight $\ell-1 / 2$ with respect to

$$
\Gamma_{0}(4)=\left\{\left[\begin{array}{ll}
a & b \\
c & d
\end{array}\right] \in S p(n, \mathbb{Z}) \mid c \equiv 0 \quad(\bmod 4)\right\}
$$

in the sense of [Ibu]. For any $f \in S_{\ell-1 / 2}\left(\Gamma_{0}(4)\right)$, put $f^{\prime}(z)=f(z / 2)$ which is an element of $S_{\ell-1 / 2}\left(\widetilde{\Gamma}, \varepsilon_{\Gamma}^{-1}\right)$ in the notation of 1.4.10. Here

$$
\Gamma=\left\{\left[\begin{array}{ll}
a & b \\
c & d
\end{array}\right] \in S p(n, \mathbb{Z}) \mid b \equiv c \equiv 0 \quad(\bmod 2)\right\}
$$

is a subgroup of $\Gamma_{0}$. Then $f \mapsto f^{\prime}$ is a $\mathbb{C}$-linear isomorphism from $S_{\ell-1 / 2}\left(\Gamma_{0}(4)\right)$ onto $S_{\ell-1 / 2}\left(\widetilde{\Gamma}, \varepsilon_{\Gamma}^{-1}\right)$ compatible with the Hecke operators.

3.3.4. Let $J_{\ell, 1}^{\text {cusp }}$ be the space of Jacobi forms of weight $\ell$ and index 1 in the sense of [Ibu]. Take any $F \in J_{\ell, 1}^{\text {cusp }}$. We can write

$$
F(z, w)=\sum_{r \in \mathbb{Z}^{n} / 2 \mathbb{Z}^{n}} f_{r}(z) \theta_{r}(z, w)
$$

with holomorphic functions $f_{r}$.

The function $F^{\prime}(z, w)=F(2 z, w)$ satisfies the conditions

1) $F^{\prime}(z, w+x z+y)=\mathbf{e}_{\infty}\left(-\frac{1}{2}\langle x, x z\rangle_{S}-\langle x, w\rangle_{S}\right) F^{\prime}(z, w)$ for all $x \in 2 \mathbb{Z}^{n}$ and $y \in \mathbb{Z}^{n}$

2) $F^{\prime}\left(\gamma(z), w(c z+d)^{-1}\right)=\operatorname{det}(c z+d)^{\ell} \mathbf{e}_{\infty}\left(\frac{1}{2}\left\langle w^{t} c, w(c z+d)^{-1}\right\rangle_{S}\right) F^{\prime}(z, w)$ for all

$$
\gamma=\left[\begin{array}{ll}
a & b \\
c & d
\end{array}\right] \in\left[\begin{array}{ll}
2 & 0 \\
0 & 1
\end{array}\right]^{-1} S p(n, \mathbb{Z})\left[\begin{array}{ll}
2 & 0 \\
0 & 1
\end{array}\right],
$$

3) $\left|F^{\prime}(z, w)\right| \operatorname{det}(\operatorname{Im} z)^{\ell / 2} \exp \left(-2 \pi\left\langle\operatorname{Im} w(\operatorname{Im} z)^{-1}, \operatorname{Im}, w\right\rangle_{S}\right)$ is bounded on $\mathfrak{H}_{n} \times$ $\mathbb{C}^{n}$.

Put

$$
F^{\prime \prime}(z, w)=\sum_{h \in \Lambda / \Lambda^{\prime}} F^{\prime}(h(z, w)) \xi_{S, \infty}(h)^{-1} \eta_{S}(h ; z, w) .
$$

Here $\Lambda^{\prime}=\left(2 \mathbb{Z}^{n} \times \mathbb{Z}^{n}\right) \times \mathbb{R}$ is a normal subgroup of $\Lambda=\left(\mathbb{Z}^{n} \times \mathbb{Z}^{n}\right) \times \mathbb{R}$ which is stable under the action of $\Gamma$. Then $F^{\prime \prime}$ is an element of $J_{\ell, S}^{\text {cusp }}\left(\Gamma_{J}, \mathbf{1}_{\Gamma}\right)$ in the notation of $\mathbf{1 . 4 . 1 0}$ with the trivial character $\mathbf{1}_{\Gamma}$ of $\Gamma$. The correspondence $F \mapsto F^{\prime \prime}$ is compatible with Hecke operators. Now we have

$$
f_{F^{\prime \prime}}(z)=2^{-n / 4} \sum_{r \in \mathbb{Z}^{n} / 2 \mathbb{Z}^{n}} f_{r}(2 z)
$$


in the notation of Theorem 1.4.10. In fact, we have

$$
\begin{aligned}
& \int_{L_{z} \backslash \mathbb{C}^{n}} F^{\prime \prime}(z, w) \overline{\vartheta_{S}(z, w)} \kappa_{S}(z, w) d_{z}(w) \\
& =\int_{L_{z} \backslash \mathbb{C}^{n}} \sum_{\lambda \in L_{z} / L_{z}^{\prime}} F^{\prime}(z, w+\lambda) \overline{\vartheta_{S}(z, w+\lambda)} \kappa_{S}(z, w+\lambda) d_{Z}(w) \\
& =\int_{L_{z}^{\prime} \backslash \mathbb{C}^{n}} F^{\prime}(z, w) \overline{\vartheta_{S}(z, w)} \kappa_{S}(z, w) d_{z}(w) \\
& =\operatorname{det}(2 \operatorname{Im} z)^{-1 / 2} \sum_{r \in \mathbb{Z}^{n} / 2 \mathbb{Z}^{n}} f_{r}(2 z)
\end{aligned}
$$

by (3.3.2.1) and the orthogonality relations (3.3.2.2). Then

$$
\sum_{r \in \mathbb{Z}^{n} / 2 \mathbb{Z}^{n}} f_{r}(2 z) \in S_{\ell-1 / 2}\left(\widetilde{\Gamma}, \varepsilon_{\Gamma}^{-1}\right)
$$

by Theorem 1.4.10, and Theorem 3.2.6 implies that the correspondence

$$
F^{\prime \prime}(z, w) \mapsto \sum_{r \in \mathbb{Z}^{n} / 2 \mathbb{Z}^{n}} f_{r}(2 z)
$$

is compatible with the Hecke operators. Finally, by 3.3.3, the correspondence

$$
F(z, w) \mapsto \sum_{r \in \mathbb{Z}^{n} / 2 \mathbb{Z}^{n}} f_{r}(4 z)
$$

is a mapping from $J_{\ell, 1}^{\text {cusp }}$ into $S_{\ell-1 / 2}\left(\Gamma_{0}(4)\right)$ which is compatible with the Hecke operators. This is the result of [Ibu] and [E-Z].

\section{Appendix A. Automorphic Forms on Locally Compact Groups}

A.1. We will recall the definition of the space of automorphic forms on a locally compact unimodular group [Tak1]. Let $G$ be a locally compact unimodular group and $K$ be a compact subgroup of $G$. Let $\Gamma$ be a closed unimodular subgroup of $G$ and $E$ be a closed subgroup of $\Gamma \cap Z(G)$ where $Z(G)$ is the center of $G$. Let $\xi$ be a continuous unitary character of $\Gamma$. Put $\chi=\left.\xi\right|_{E}$. Let $\pi$ (resp. $\delta$ ) be an irreducible unitary representation of $G$ (resp. $K$ ) with representation space $H_{\pi}$ (resp. $V_{\delta}$ ). We shall suppose the following two conditions:

(A) $\left.\pi\right|_{E}=\chi$, that is, $\pi(a)$ is equal to the multiplication by $\chi(a)$ for all $a \in E$,

(B) the multiplicity of $\delta$ in $\left.\pi\right|_{K}$ is equal to one.

A.2. The space $\check{\mathcal{A}}_{\delta}(\Gamma \backslash G, \xi, \pi)$ of the automorphic forms on $G$ associated with the data $(G, K, \Gamma, E ; \xi, \pi, \delta)$ consists of the continuous $V_{\delta}$-valued functions $f$ on $G$ such that

1) $f(\gamma x)=\xi\left(\gamma^{-1}\right) f(x)$ for all $\gamma \in \Gamma$,

2) $\int_{\Gamma \backslash G}|f(x)|^{2} d(\dot{x})<\infty$

3) $f(x k)=\delta\left(k^{-1}\right) f(x)$ for all $k \in K$,

4) $\int_{G / E} f\left(x y^{-1}\right) \varphi(y) d(\dot{y})=\widehat{\psi}_{\pi, \delta}(\varphi) f(x)$ for all $\varphi \in C_{c}(G / E, \chi, \delta)^{0}$.

Here $C_{c}(G / E, \chi, \delta)^{0}$ is an involutive $\mathbb{C}$-algebra consisting of the $\mathbb{C}$-valued continuous functions $\varphi$ on $G$ such that 
i) $\varphi(a x)=\chi\left(a^{-1}\right) \varphi(x)$ for all $a \in E$,

ii) $\operatorname{supp}(\varphi)$ is compact modulo $E$,

iii) $\varphi\left(k x k^{-1}\right)=\varphi(x)$ for all $k \in K$,

iv) $\int_{K} e_{\delta}(k) \varphi\left(k^{-1} x\right) d k=\varphi(x)$ where $e_{\delta}(k)=(\operatorname{dim} \delta) \overline{\operatorname{tr} \delta(k)}$.

The Haar measure on $K$ is normalized so that $\operatorname{vol}(K)=1$. The multiplication is defined by the convolution

$$
\varphi * \psi(x)=\int_{G / E} \varphi\left(x y^{-1}\right) \psi(y) d(\dot{y}) \quad \text { for } \varphi, \psi \in C_{c}(G / E, \chi, \delta)^{0} .
$$

The involution on $C_{c}(G / E, \chi, \delta)^{0}$ is defined by $\varphi^{*}(x)=\overline{\varphi\left(x^{-1}\right)}$. Put

$$
\widehat{\psi}_{\pi, \delta}(\varphi)=(\operatorname{dim} \delta)^{-1} \int_{G / E} \varphi(x) \psi_{\pi, \delta}(x) d(\dot{x}) \quad \text { for } \varphi \in C_{c}(G / E, \chi, \delta)^{0},
$$

where $\psi_{\pi, \delta}(x)=\operatorname{tr}(P \circ \pi(x) \circ P)(x \in G)$ with the orthogonal projection $P$ of $H_{\pi}$ onto the $\delta$-isotypic component of $H_{\pi}$.

The space $\tilde{\mathcal{A}}_{\delta}(\Gamma \backslash G, \xi, \pi)$ is a complex Hilbert space with respect to the inner product

$$
(f, g)=\int_{\Gamma \backslash G}(f(x), g(x))_{\delta} d(\dot{x})
$$

where $(,)_{\delta}$ is the Hermitian inner product of $V_{\delta}$.

A.3. Let $\check{\pi}($ resp. $\check{\delta}$ ) be the contragredient representation of $\pi$ (resp. $\delta$ ). We will denote by $\operatorname{Ind}\left(G, \Gamma ; \xi^{-1} ; \check{\pi}\right)$ the $\check{\pi}$-isotypic component of the representation $\operatorname{Ind}\left(G, \Gamma ; \xi^{-1}\right)$. Let us denote by $\operatorname{Ind}\left(G, \Gamma ; \xi^{-1} ; \check{\pi}, \check{\delta}\right)$ the $\check{\delta}$-isotypic component of $\operatorname{Ind}\left(G, \Gamma ; \xi^{-1} ; \check{\pi}\right)$. Then we have a $\mathbb{C}$-linear isometry

$$
\check{\mathcal{A}}_{\delta}(\Gamma \backslash G, \xi, \pi) \otimes_{\mathbb{C}} V_{\delta}^{*} \stackrel{\sim}{\rightarrow} \operatorname{Ind}\left(G, \Gamma ; \xi^{-1} ; \check{\pi}, \check{\delta}\right)
$$

defined by $f \otimes \alpha \mapsto(\operatorname{det} \delta)^{1 / 2}\langle f, \alpha\rangle$. Here $V_{\delta}^{*}$ is the complex dual space of $V_{\delta}$ with the canonical pairing $\langle\rangle:, V_{\delta} \times V_{\delta}^{*} \rightarrow \mathbb{C}$. In particular, the dimension of $\check{\mathcal{A}}_{\delta}(\Gamma \backslash G, \xi, \pi)$ is equal to the multiplicity of $\pi$ in $\operatorname{Ind}(G, \Gamma ; \xi)$.

A.4. Put $C_{c}(G, \delta)^{0}=C_{c}(G / E, \chi, \delta)^{0}$ with $E=\{1\}$. Put

$$
\varphi_{\chi}(x)=\int_{E} \varphi(x a) \chi(a) d a \quad \text { for all } \varphi \in C_{c}(G, \delta)^{0} .
$$

We have a surjective involutive $\mathbb{C}$-algebra homomorphism $\varphi \mapsto \varphi_{\chi}$ of $C_{c}(G, \delta)^{0}$ to $C_{c}(G, \chi, \delta)^{0}$. The condition 4$)$ of the definition of $\check{\mathcal{A}}_{\delta}(\Gamma \backslash G, \xi, \pi)$ is equivalent to the condition

$\left.4^{\prime}\right) \int_{G} f\left(x y^{-1}\right) \varphi(y) d y=\widehat{\psi}_{\pi, \delta}(\varphi) f(x)$ for all $\varphi \in C_{c}(G, \delta)^{0}$.

Here we put

$$
\widehat{\psi}_{\pi, \delta}(\varphi)=(\operatorname{dim} \delta)^{-1} \int_{G} \varphi(x) \psi_{\pi, \delta}(x) d x
$$


A.5. Let us consider the restricted direct product. Let $\left\{G_{p}\right\}_{p \in P}$ be a family of locally compact unimodular groups with a countable index set $P$. We shall suppose that there exists a finite subset $P_{\infty}$ of $P$ such that $G_{p}$ is totally disconnected for all $p \notin P_{\infty}$. Let $K_{p}$ be a compact subgroup of $G_{p}(p \in P)$ such that $K_{p}$ is open in $G_{p}$ for all $p \notin P_{\infty}$. Let $E_{p}$ be a closed subgroup of $Z\left(G_{p}\right)$.

Let $G$ be the restricted direct product of $\left\{G_{p}\right\}_{p \in P}$ with respect to $\left\{K_{p}\right\}_{p \in P}$. Then $K=\prod_{p \in P} K_{p}$ is a compact subgroup of $G$.

$$
Z(G)=G \cap \prod_{p \in P} Z\left(G_{p}\right) \quad\left(\text { resp. } E=G \cap \prod_{p \in P} E_{p}\right)
$$

is the restricted direct product of $\left\{Z\left(G_{p}\right)\right\}_{p \in P}$ (resp. $\left\{E_{p}\right\}_{p \in P}$ ) with respect to $\left\{Z\left(G_{p}\right) \cap K_{p}\right\}_{p \in P}$ (resp. $\left\{E_{p} \cap K_{p}\right\}_{p \in P}$ ). Let $\Gamma$ be a closed unimodular subgroup of $G$ and $\xi$ be a continuous unitary character of $\Gamma$. Put $\chi=\left.\xi\right|_{E}$.

Let us denote by $\widehat{E}_{p}$ the Pontryagin dual of $E_{p}$. Let $L_{p}$ be a subgroup of $\widehat{E}_{p}$ consisting of the $\alpha \in \widehat{E}_{p}$ such that $\alpha\left(E_{p} \cap K_{p}\right)=1$. Then $L_{p}$ is an open compact subgroup of $\widehat{E}_{p}$ for all $p \notin P_{\infty}$. For any element $\left(\alpha_{p}\right)_{p \in P}$ of the restricted direct product $\prod_{p \in P}^{\prime} \widehat{E}_{p}$ with respect to $\left\{L_{p}\right\}_{p \in P}$, define a character $\bigotimes_{p \in P} \alpha_{p}$ of $E$ by

$$
\bigotimes_{p \in P} \alpha_{p}(a)=\prod_{p \in P} \alpha_{p}\left(a_{p}\right) \quad \text { for all } a=\left(a_{p}\right)_{p \in P} \in E .
$$

Then $\widehat{E}$ is identified with $\prod_{p \in P}^{\prime} \widehat{E}_{p}$ by $\bigotimes_{p \in P} \alpha_{p}=\left(\alpha_{p}\right)_{p \in P}$. So we have $\chi=$ $\bigotimes_{p \in P} \chi_{p}$ with a suitable $\chi_{p} \in \widehat{E}_{p}$.

Let $\delta$ be an irreducible unitary representation of $K$. Then there exists a finite subset $S$ of $P$ containing $P_{\infty}$ and an irreducible unitary representation $\delta_{p}$ of $K_{p}$ for all $p \in S$ such that

$$
\delta=\left(\bigotimes_{p \in S} \delta_{p}\right) \otimes\left(\text { trivial representation of } \prod_{p \notin S} K_{p}\right) .
$$

For all $p \notin S$, put $\delta_{p}=\mathbf{1}_{K_{p}}$, the trivial representation of $K_{p}$.

Let $\pi_{p}$ be an irreducible unitary representation of $G_{p}$ on the representation space $H_{p}$ for each $p \in P$ such that

1) $\left.\pi_{p}\right|_{E_{p}}=\chi_{p}$ for all $p \in P$,

2) for all $p \notin S$, the multiplicity of $\delta_{p}=\mathbf{1}_{K_{p}}$ in $\left.\pi_{p}\right|_{K_{p}}$ is equal to one.

Choose, for each $p \notin S$, a $K_{p}$-invariant vector $v_{p} \in H_{p}$ of unit length. Let us denote by $\bigotimes_{p \in P}^{\prime} H_{p}$ the $\mathbb{C}$-linear span of

$$
\left\{\bigotimes_{p \in P} u_{p} \in \bigotimes_{p \in P} H_{p} \mid u_{p}=v_{p} \text { for almost all } p \in P \backslash S\right\}
$$

in $\bigotimes_{p \in P} H_{p}$. The $\mathbb{C}$-vector space $\bigotimes_{p \in P}^{\prime} H_{p}$ is a pre-Hilbert space with respect to the inner product defined by

$$
\left(\bigotimes_{p \in P} u_{p}, \bigotimes_{p \in P} u_{p}^{\prime}\right)=\prod_{p \in P}\left(u_{p}, u_{p}^{\prime}\right) \quad \text { for } \bigotimes_{p \in P} u_{p}, \bigotimes_{p \in P} u_{p}^{\prime} \in \bigotimes_{p \in P}^{\prime} H_{p}
$$

The completion $\widehat{\bigotimes}_{p \in P}^{\prime} H_{p}$ of $\bigotimes_{p \in P}^{\prime} H_{p}$ with respect to the inner product is called the completed restricted tensor product of $\left\{H_{p}\right\}_{p \in P}$ with respect to $\left\{v_{p}\right\}_{p \in P \backslash S}$. 
Now the group $G$ acts on $\bigotimes_{p \in P}^{\prime} H_{p}$ by

$$
g \cdot \bigotimes_{p \in P} u_{p}=\bigotimes_{p \in P} \pi_{p}\left(g_{p}\right) u_{p} \quad \text { for } g=\left(g_{p}\right)_{p \in P} \in G, \bigotimes_{p \in P} u_{p} \in \bigotimes_{p \in P}^{\prime} H_{p}
$$

This action is unitary. So the action is extended continuously to the completion $\widehat{\bigotimes}_{p \in P}^{\prime} H_{p}$, which is called the (completed) restricted tensor product of $\left\{\pi_{p}\right\}_{p \in P}$ with respect to $\left\{v_{p}\right\}_{p \in P \backslash S}$. Then the (completed) restricted tensor product $\pi=\bigotimes_{p \in P}^{\prime} \pi_{p}$ with respect to $\left\{v_{p}\right\}_{p \in P \backslash S}$ is an irreducible unitary representation of $G$ such that $\left.\pi\right|_{E}=\chi$. If $C_{c}\left(G_{p} / E_{p}, \chi_{p}, \mathbf{1}_{K_{p}}\right)^{0}$ is commutative for almost all $p \in P$ and $G_{p}$ is of type I for all $p \in P$, then all irreducible unitary representations $\pi$ of $G$ such that $\left.\pi\right|_{E}=\chi$ are constructed as above (see $\S 3.2$ of [God]).

For any $p \notin S$, let $\psi_{p}^{0}$ be the characteristic function of $K_{p}$ in $G_{p}$. Then $\varphi_{p}^{0}=\theta_{\chi_{p}}\left(\psi_{p}^{0}\right)$ in the notation of $\mathbf{B . 2}$ is the unity of $C_{c}\left(G_{p} / E_{p}, \chi_{p}, \mathbf{1}_{K_{p}}\right)^{0}$. The algebraic restricted tensor product $\bigotimes_{p \in P}^{\prime} C_{c}\left(G_{p} / E_{p}, \chi_{p}, \delta_{p}\right)^{0}$ with respect to $\left\{\varphi_{p}^{0}\right\}_{p \notin S}$ is identified with a subalgebra of $C_{c}(G / E, \chi, \delta)^{0}$ by

$$
\left(\bigotimes_{p \in P} \varphi_{p}\right)(x)=\prod_{p \in P} \varphi_{p}\left(x_{p}\right) \quad \text { for all } x=\left(x_{p}\right)_{p \in P} \in G \text {. }
$$

Then $\bigotimes_{p \in P}^{\prime} C_{c}\left(G_{p} / E_{p}, \chi_{p}, \delta_{p}\right)^{0}$ is dense in $C_{c}(G / E, \chi, \delta)^{0}$ with respect to the $L^{1}$ norm

$$
|\varphi|=\int_{G / E}|\varphi(x)| d \dot{x} \quad \text { for } \varphi \in C_{c}(G / E, \chi, \delta)^{0} .
$$

The action of $C_{c}(G / E, \chi, \delta)^{0}$ on the representation space of any unitary representation of $G$ is continuous with respect to the $L^{1}$-norm. So it is enough to consider the algebraic restricted tensor product of $\left\{C_{c}\left(G_{p} / E_{p}, \chi_{p}, \delta_{p}\right)^{0}\right\}_{p \in P}$. Then we have

Proposition. Suppose that the multiplicity of $\delta$ in $\left.\pi\right|_{K}$ is equal to one (that is, the multiplicity of $\delta_{p}$ in $\left.\pi_{p}\right|_{K_{p}}$ is equal to one for all $p \in P$ ). Then the space $\check{\mathcal{A}}_{\delta}(\Gamma \backslash G, \xi, \pi)$ of the automorphic forms associated with the data $(G, K, \Gamma, E ; \xi, \pi, \delta)$ consists of the continuous $V_{\delta}$-valued functions $f$ on $G$ such that

1) $f(\gamma x)=\xi\left(\gamma^{-1}\right) f(x)$ for all $\gamma \in \Gamma$.

2) $\int_{\Gamma \backslash G}|f(x)|^{2} d \dot{x}<\infty$.

3) $f(x k)=\delta\left(k^{-1}\right) f(x)$ for all $k \in K$.

4) $f$ satisfies the integral equations

$$
\int_{G_{\infty} / E_{\infty}} f\left(x y^{-1}\right) \prod_{p \in P_{\infty}} \varphi_{p}(y) d \dot{y}=\prod_{p \in P_{\infty}} \widehat{\psi}_{\pi_{p}, \delta_{p}}\left(\varphi_{p}\right) \cdot f(x)
$$

for all $\varphi_{p} \in C_{c}\left(G_{p} / E_{p}, \chi_{p}, \delta_{p}\right)^{0}$ with all $p \in P_{\infty}$. Here we put

$$
G_{\infty}=\prod_{p \in P_{\infty}} G_{p}, \quad E_{\infty}=\prod_{p \in P_{\infty}} E_{p} .
$$

5) $\int_{G_{p} / E_{p}} f\left(x y^{-1}\right) \varphi(y) d \dot{y}=\widehat{\psi}_{\pi_{p}, \delta_{p}}(\varphi) f(x)$ for all $\varphi \in C_{c}\left(G_{p} / E_{p}, \chi_{p}, \delta_{p}\right)^{0}$ with all $p \in P \backslash P_{\infty}$. 


\section{Appendix B. Zonal Spherical Functions with a Central Character}

B.1. In this appendix, we will recall some basic facts on the zonal spherical functions on a locally compact group and fix some notation for our use.

Let $G$ be a locally compact unimodular group, $K$ be a compact subgroup of $G$ and $E$ be a closed subgroup of the center of $G$. Let $\chi$ be a continuous unitary character of $E$. Let us denote by $\widehat{G}$ the set of the unitary equivalence classes of the irreducible unitary representations of $G$. The Haar measure on $K$ is normalized so that $\operatorname{vol}(K)=1$.

B.2. Let $C_{c}(G, E ; \chi)$ be the involutive $\mathbb{C}$-algebra consisting of the continuous $\mathbb{C}$ valued functions $\varphi$ on $G$ such that

1) $\varphi$ is compactly supported modulo $E$, and

2) $\varphi(x a)=\chi\left(a^{-1}\right) \varphi(x)$ for all $a \in E$,

with the convolution product

$$
(\varphi * \psi)(x)=\int_{G / E} \varphi\left(x y^{-1}\right) \psi(y) d \dot{y}
$$

and the involution $\varphi^{*}(x)=\overline{\varphi\left(x^{-1}\right)}$. Let us denote by $C_{c}(G / / K, E ; \chi)$ the involutive $\mathbb{C}$-subalgebra of $C_{c}(G, E ; \chi)$ consisting of the $K$-biinvariant $\varphi$, that is, $\varphi\left(k x k^{\prime}\right)=$ $\varphi(x)$ for all $k, k^{\prime} \in K$. With the notation of $\mathbf{A . 2}$, we have

$$
C_{c}(G / / K, E ; \chi)=C_{c}\left(G / E, \chi, \mathbf{1}_{K}\right)^{0}
$$

where $\mathbf{1}_{K}$ is the trivial representation of $K$.

For any compact subset $M$ of $G$, let $C_{M}(G, E ; \chi)$ be the subspace of $C_{c}(G, E ; \chi)$ consisting of the $\varphi$ such that $\operatorname{supp}(\varphi) \subset M E$, which is a complex Banach space with respect to the norm $|\varphi|=\sup _{x \in G}|\varphi(x)|$. Endowed with the injective limit topology, $C_{c}(G, E ; \chi)=\varliminf_{M} C_{M}(G, E ; \chi)$ is a locally convex space.

Put $C_{c}(G)=C_{c}(G,\{1\} ; \mathbf{1})$ and $C_{c}(G / / K)=C_{c}(G / / K,\{1\} ; \mathbf{1})$. Put

$$
\varphi_{\chi}(x)=\int_{E} \varphi(x a) \chi(a) d a \text { for all } \varphi \in C_{c}(G) .
$$

Then the mapping $\theta_{\chi}: \varphi \mapsto \varphi_{\chi}$ is a continuous surjective involutive $\mathbb{C}$-algebra homomorphism from $C_{c}(G)$ to $C_{c}(G, E ; \chi)$ such that $\theta_{\chi} C_{c}(G / / K)=C_{c}(G / / K, E ; \chi)$.

B.3. Let $\Omega(G / / K)$ be the set of the zonal spherical functions on $G$ with respect to $K$, that is, the set of the continuous $\mathbb{C}$-valued functions $\omega$ on $G$ satisfying the following equivalent conditions:

1) $\omega$ is $K$-biinvariant with $\omega(1)=1$ and $\varphi * \omega=\lambda_{\varphi} \omega$ for all $\varphi \in C_{c}(G / / K)$ with $\lambda_{\varphi} \in \mathbb{C}$,

2) $\int_{K} \omega(x k y) d k=\omega(x) \omega(y)$ for all $x, y \in G$, and $\omega \neq 0$,

3) $\widehat{\omega}: C_{c}(G / / K) \rightarrow \mathbb{C}$ is a surjective $\mathbb{C}$-algebra homomorphism where

$$
\widehat{\omega}(\varphi)=\int_{G} \varphi(x) \omega(x) d x \quad \text { for } \varphi \in C_{c}(G / / K) .
$$

Let $\Omega(G / / K, E ; \chi)$ be the subset of $\Omega(G / / K)$ consisting of the $\omega$ satisfying the following equivalent conditions:

i) $\omega(x a)=\chi(a) \omega(x)$ for all $a \in E$, 
ii) there exists a $\mathbb{C}$-algebra homomorphism

$$
\alpha: C_{c}(G / / K, E ; \chi) \rightarrow \mathbb{C} \text { such that } \widehat{\omega}=\alpha \circ \theta_{\chi} .
$$

For an $\omega \in \Omega(G / / K, E ; \chi)$, put

$$
\widehat{\omega}(\varphi)=\int_{G / E} \varphi(x) \omega(x) d \dot{x} \quad \text { for all } \varphi \in C_{c}(G / / K, E ; \chi) .
$$

Then $\widehat{\omega}: C_{c}(G / / K, E ; \chi) \rightarrow \mathbb{C}$ is a continuous surjective $\mathbb{C}$-algebra homomorphism such that $\omega\left(\varphi_{\chi}\right)=\omega(\varphi)$ for all $\varphi \in C_{c}(G / / K)$. Conversely, for any continuous surjective $\mathbb{C}$-algebra homomorphism $\lambda: C_{c}(G / / K, E ; \chi) \rightarrow \mathbb{C}$, there exists uniquely an $\omega \in \Omega(G / / K, E ; \chi)$ such that $\widehat{\omega}=\lambda$.

If $K$ is an open subgroup of $G$, then the last statement is valid without continuity condition on $\lambda$ [Tam]. In this case, $C_{c}(G / / K)$ is a $\mathbb{C}$-algebra with unity $\varphi_{K}$ the characteristic function of $K$ in $G$. Then $C_{c}(G / / K, E ; \chi)$ also has a unity $\theta_{\chi}\left(\varphi_{K}\right)$.

B.4. Let us denote by $\mathcal{R}(G, E ; \chi)$ the subset of $\widehat{G}$ consisting of the $\pi$ such that $\left.\pi\right|_{E}=\chi$ and by $\mathcal{R}(G / / K, E ; \chi)$ the subset of $\mathcal{R}(G, E ; \chi)$ consisting of the $\pi$ with non-trivial $K$-invariant vectors. If the algebra $C_{c}(G / / K, E ; \chi)$ is commutative, then for any $\pi \in \mathcal{R}(G / / K, E ; \chi)$, the space of the $K$-invariant vectors is one-dimensional. The following two conditions are equivalent:

1) $C_{c}(G / / K)$ is a commutative $\mathbb{C}$-algebra,

2) for any $\pi \in \widehat{G}$, the space of the $K$-invariant vectors of $\pi$ is at most onedimensional.

Suppose $C_{c}(G / / K, E ; \chi)$ is commutative. Let us denote by $\Omega^{+}(G / / K, E ; \chi)$ the subset of $\Omega(G / / K, E ; \chi)$ consisting of the positive $\omega$, that is, the Hermitian matrix $\left(\omega\left(x_{i} x_{j}^{-1}\right)\right)_{i, j}$ is positive definite for all finite subsets $\left\{x_{1}, \cdots, x_{n}\right\}$ of $G$. For any $\pi \in \mathcal{R}(G / / K, E ; \chi)$, take a $K$-invariant vector $v$ with unit length and put $\omega_{\pi}(x)=(\pi(x) v, v)(x \in G)$. Then $\pi \mapsto \omega_{\pi}$ is a bijection from $\mathcal{R}(G / / K, E ; \chi)$ onto $\Omega^{+}(G / / K, E ; \chi)$.

Any $\omega \in \Omega^{+}(G / / K, E ; \chi)$ is a bounded function:

$$
|\omega(x)| \leq \omega(1) \quad \text { for all } x \in G .
$$

Let us denote by $\Omega^{b}(G / / K, E ; \chi)$ the subset of $\Omega(G / / K, E ; \chi)$ consisting of the bounded functions.

\section{REFERENCES}

[E-Z] M.Eichler and D.Zagier, Theory of Jacobi Forms, Progress in Math. 55, 1985. MR $\mathbf{8 6 j}: 11043$

[God] Godement,R., Notes on Jacquet-Langlands' theory, The Institute for Advanced Study, 1970.

[H-S] Hecht,H. and Schmid,W., On Integrable Representations of a Semisimple Lie Group, Math.Ann. 220 (1976), 147-149. MR 53:3203

[Ibu] Ibukiyama,T., On Jacobi forms and Siegel modular forms of half integral weights, Comment. Math. Univ. St. Pauli 41 (1992), 109-124. MR 94a:11069

[Igu] Igusa,J.-I., Harmonic analysis and theta-functions, Acta Math. 120 (1968), 187-222. MR 43:2428

[Knp] Knapp,A.W., Representation Theory of Semisimple Groups, Princeton Univ. Press, 1986. MR 87j:22022

$[\mathrm{K}-\mathrm{V}]$ Kashiwara,M. and Vergne,M., On the Segal-Shale-Weil Representations and Harmonic Polynomials, Inv.Math. 44 (1978), 1-47. MR 57:3311 
[Kug] Kuga,M., Fibre varieties over a symmetric space whose fibres are abelian varieties., Algebraic Groups and Discontinuous Subgroups P. S. P. M. vol. 9 (Amer. Math. Soc.) (1966), 338-346. MR 34:5990

[Mum] Mumford,D., Families of abelian varieties, Algebraic Groups and Discontinuous Subgroups P.S.P.M. vol.9 (Amer.Math.Soc.) (1966), 347-351. MR 34:5828

[Mur] Murase,A., L-functions attached to Jacobi forms of degree n, Part I. The basic identity, J. reine angew. Math. 401 (1989), 122-156. MR 90k:11064

[Sat1] Satake,I., Factors of Automorphy and Fock Representations, Advances in Math. 7 (1971), 83-110. MR 50:541

[Sat2] Satake,I., Unitary representations of semi-direct product of Lie groups of $\bar{\partial}$-cohomology, Math. Ann. 190 (1971), 177-202. MR 45:5274

[Sat3] Satake,I., Theory of spherical functions on reductive algebraic groups over p-adic fields, Publ. Math., I.H.E.S. 18 (1963), 5-69. MR 33:4059

[Sat4] Satake,I., Holomorphic imbedding of symmetric domains into Siegel space, Amer. J. Math. 87 (1965), 425-461. MR 33:4326

[Sat5] Satake,I., Algebraic Structures of Symmetric Domains, Iwanami Shoten and Princeton Univ. Press, 1980. MR 82i:32003

[Shn] Shintani,T., unpublished note.

[Tak1] Takase,K., A note on automorphic forms, J.reine angew.Math. 409 (1990), 138-171. MR 91h: 11040

[Tak2] Takase,K., On unitary representation of Jacobi group, J. reine angew. Math. 430 (1992), 139-149 (Correction: J. reine angew. Math. 441 (1993), 215-216). MR 93e:11064; CMP 93:15

[Tak3] Takase,K., On Two-fold covering group of $S p(n, \mathbb{R})$ and automorphic factor of weight $1 / 2$, Comment. Math. Univ. Sancti Pauli 45 (1996), 117-145. MR 98c:11041

[Tam] Tamagawa,T., On Selberg's trace formula, J.Fac.Sci.Univ.Tokyo 8 (1960), 363-386. MR 23:A958

[Wei] Weil,A., Sur certains groupes d'opérateurs unitaires, Acta Math. 111 (1964), 143-211. MR 29:2324

Department of Mathematics, Miyagi University of Education, Aoba-ku, Sendai 980, JAPAN

E-mail address: f26508@cctu.cc.tohoku.ac.jp 